\title{
Implementation of Laterally Loaded Piles in Multi-Layer Soils
}

JTRP SPR- 3261

Final SAC meeting

SAC members

Mir Zaheer and Keith Hoernschemeyer

\section{Purdue University}




\section{Introduction}

-Analysis developed for the design of laterally loaded piles in multi-layer soil using energy principles (SPR 2630)

-Analysis validation for piles in sand

- Model pile lateral load tests

(single piles and pile groups)

-Driven

- Preinstalled

- Jacked 


\section{Objectives}

-Study the response of piles subjected to lateral load through a series of model pile load tests

-Evaluate the effect of pile installation (driven, jacked and preinstalled) on pile response

-Compare the model pile experimental results with results from the analysis for preinstalled model piles 


\section{General scope of work}

Task 1 Purchase of sensors

Task 2 Fabrication of piles and pile caps

Task 3 Fabrication of jacking system

Task 4 Performance of model pile load tests

Task 5 Validation of the analysis 


\section{Contents}

- Model pile testing plan

- Model pile testing

- Sample preparation

- Soil tank

- Sand pluviator

- Instrumented model pile

- Driving system

- Preinstallation method

- Jacking system

- Installation of piles for pile group testing

- Lateral loading system

- Comparison of test and analysis results

- Single piles

- Pile groups

- Summary and Conclusions 


\section{Model pile testing plan}

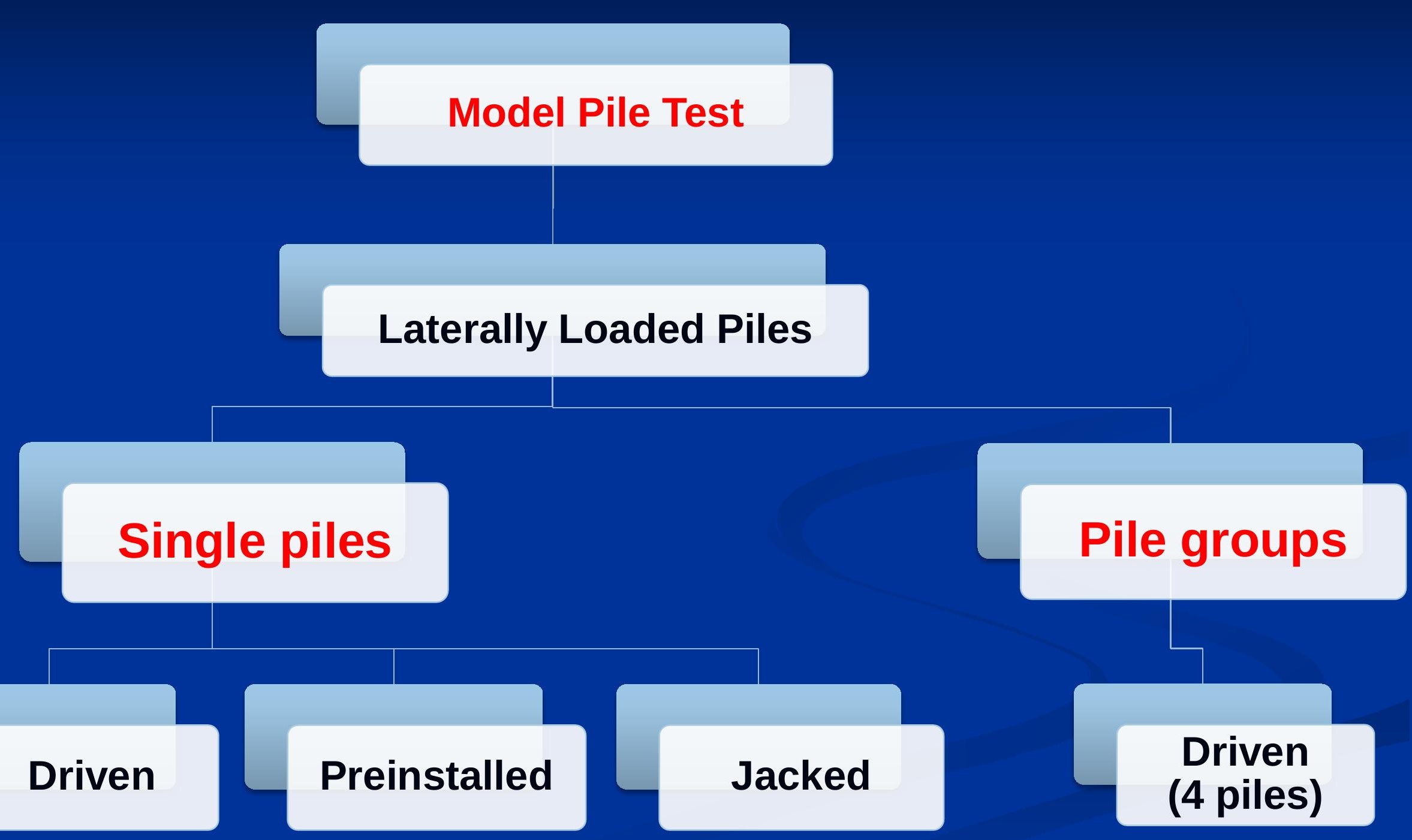




\section{Model pile testing plan}

\begin{tabular}{|c|c|c|c|}
\hline Pile & Installation & Soil Condition & Relative Density \\
\hline \multirow{9}{*}{ Single pile } & \multirow{4}{*}{ Driven } & Dense sand & $D_{R} \approx 90 \%$ \\
\hline & & Medium dense sand & $D_{R} \approx 60 \%$ \\
\hline & & Loose sand & $D_{R} \approx 40 \%$ \\
\hline & & Multi-layer & $\mathrm{D}_{\mathrm{R}} \approx 60 \%, 90 \%$ \\
\hline & \multirow{2}{*}{ Preinstalled } & Dense sand & $\mathrm{D}_{\mathrm{R}} \approx 90 \%$ \\
\hline & & Medium dense sand & $D_{R} \approx 60 \%$ \\
\hline & \multirow{3}{*}{ Jacked } & Dense sand & $D_{R} \approx 90 \%$ \\
\hline & & Medium dense sand & $D_{R} \approx 60 \%$ \\
\hline & & Loose sand & $D_{R} \approx 40 \%$ \\
\hline \multirow{4}{*}{ Group piles } & \multirow{4}{*}{ Driven } & Dense sand & $D_{R} \approx 90 \%$ \\
\hline & & Medium dense sand & $D_{R} \approx 60 \%$ \\
\hline & & Loose sand & $D_{R} \approx 40 \%$ \\
\hline & & Multi-layer & $\mathrm{D}_{\mathrm{R}} \approx 60 \%, 90 \%$ \\
\hline
\end{tabular}




\section{Contents}

- Model pile testing plan

- Model pile testing

- Sample preparation

- Soil tank

- Sand pluviator

- Instrumented model pile

- Driving system

- Preinstallation method

- Jacking system

- Installation of piles for pile group testing

- Lateral loading system

- Comparison of test and analysis results

- Single piles

- Pile groups

- Summary and Conclusions 


\section{Soil tank}

\section{- Soil tank ( $D=2 m, H=1.6 m)$}

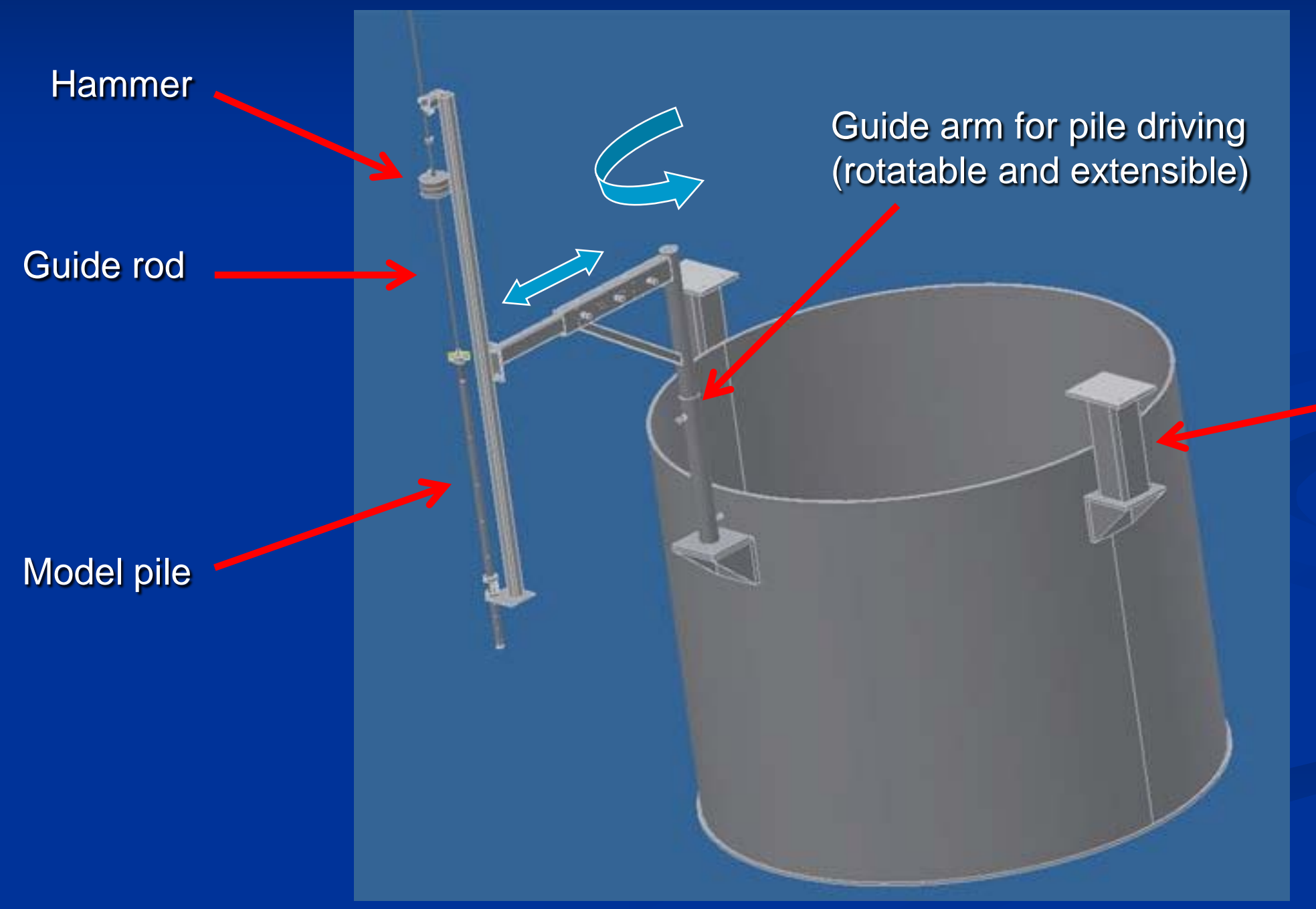

Reaction beam support 


\section{Soil tank}

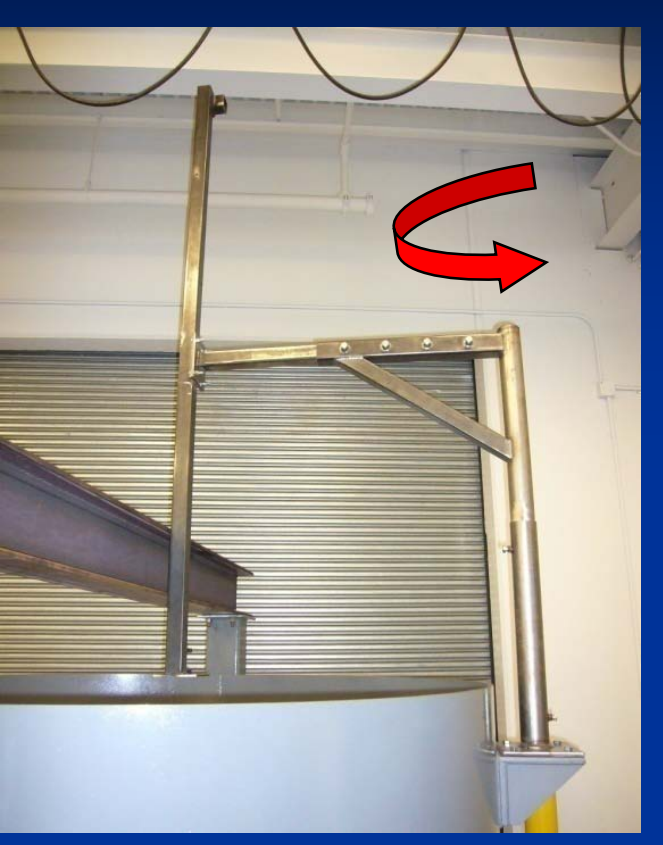

Guide arm for pile driving

Reaction

beam

(detachable)

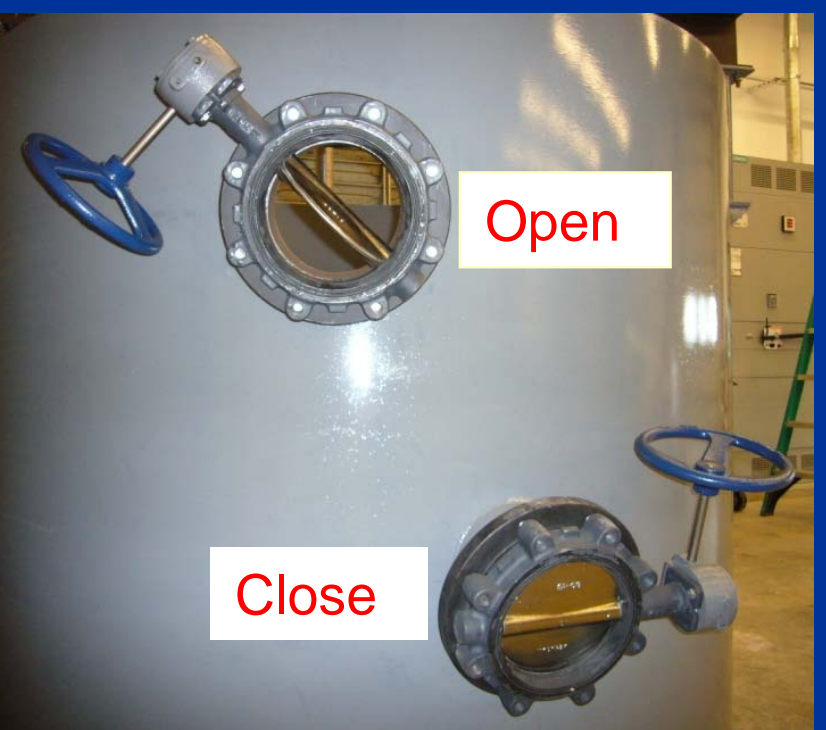

Holes for draining sand

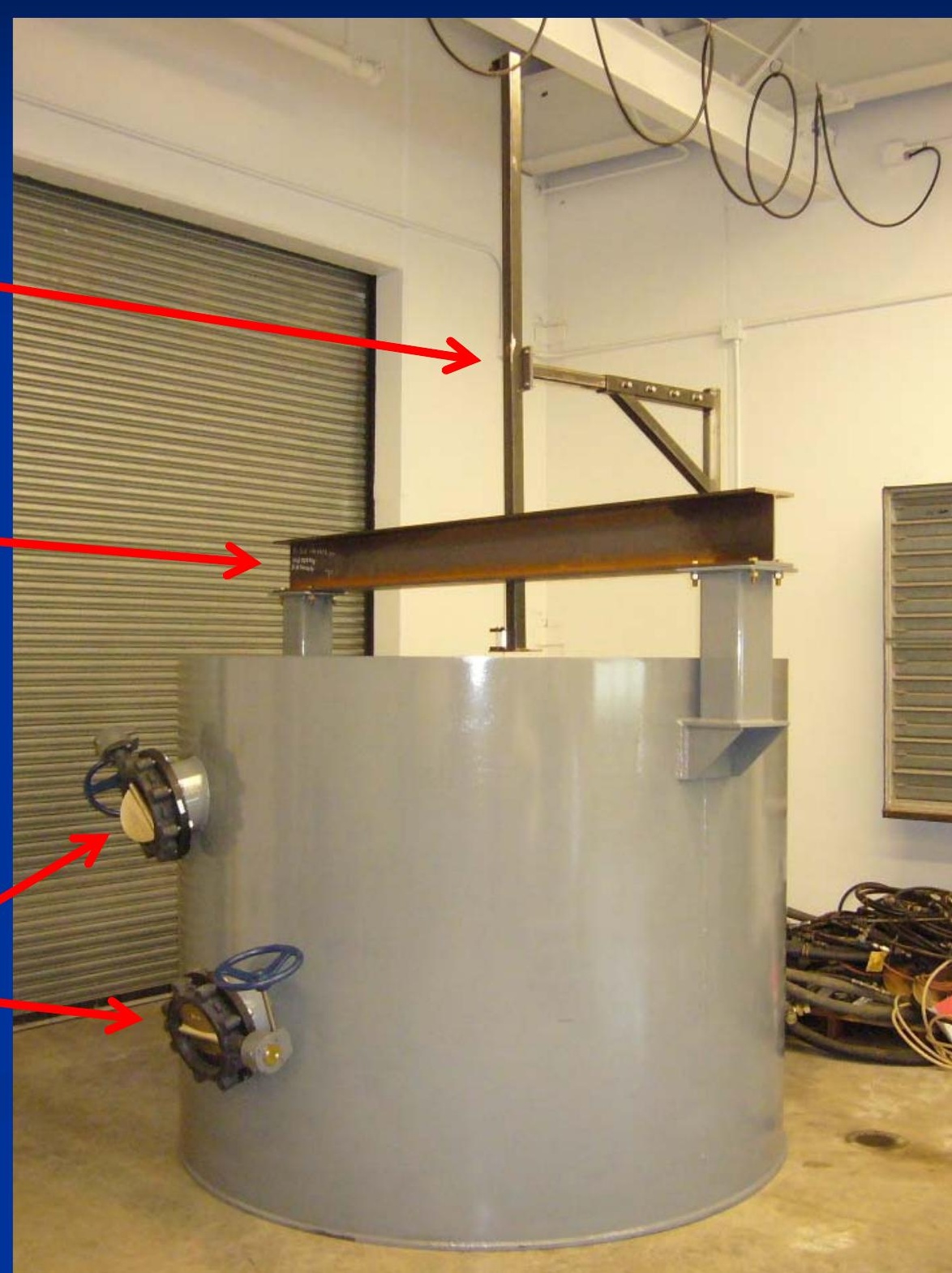




\section{Sand pluviator}

- Large-scale sand pluviation system (D=2m)

Diffuser

sieves

Soil tank

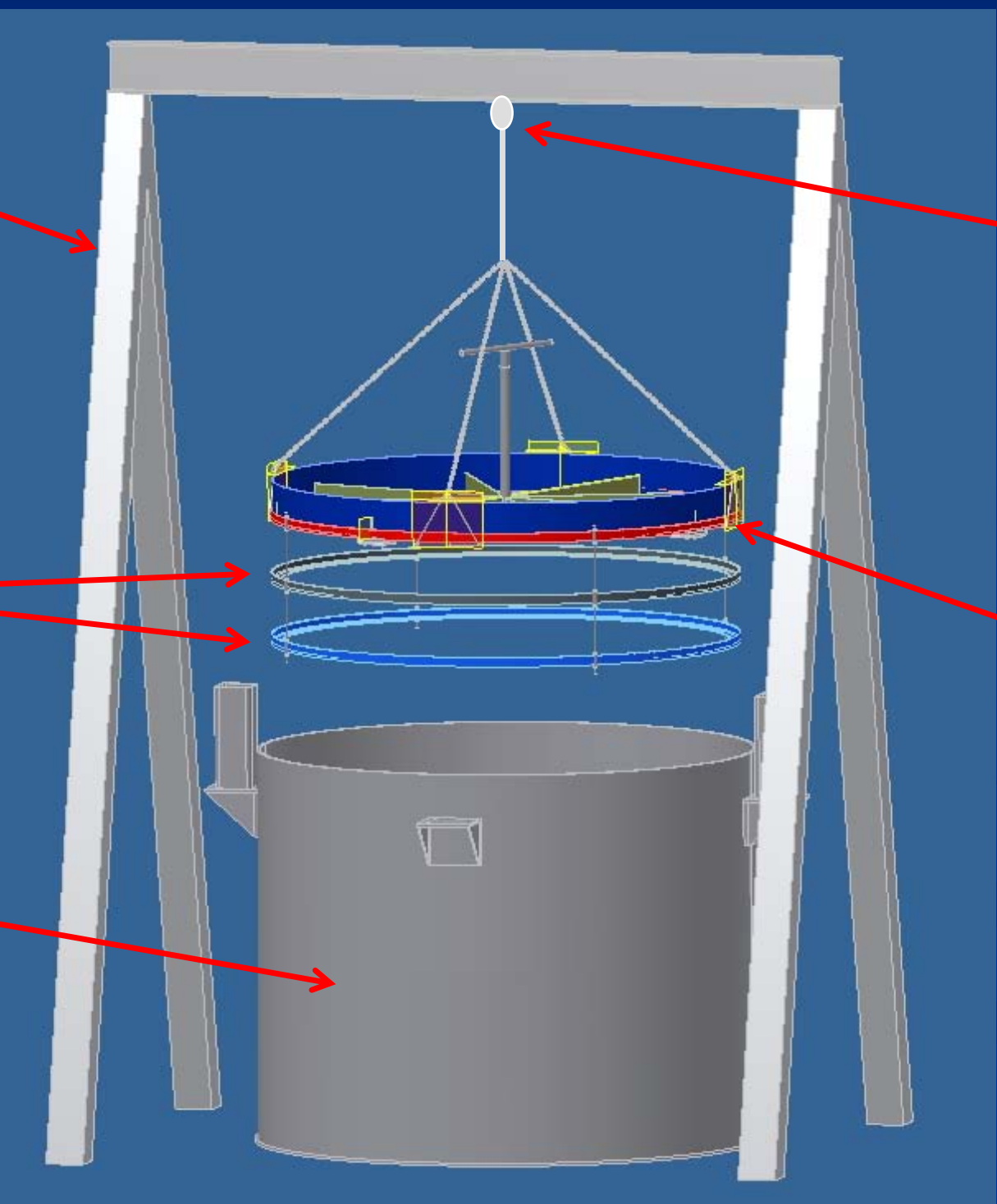

Hoist for

adjusting height

Shutter plate 


\section{Sand pluviator}

Top of pluviator

Two sieve layers
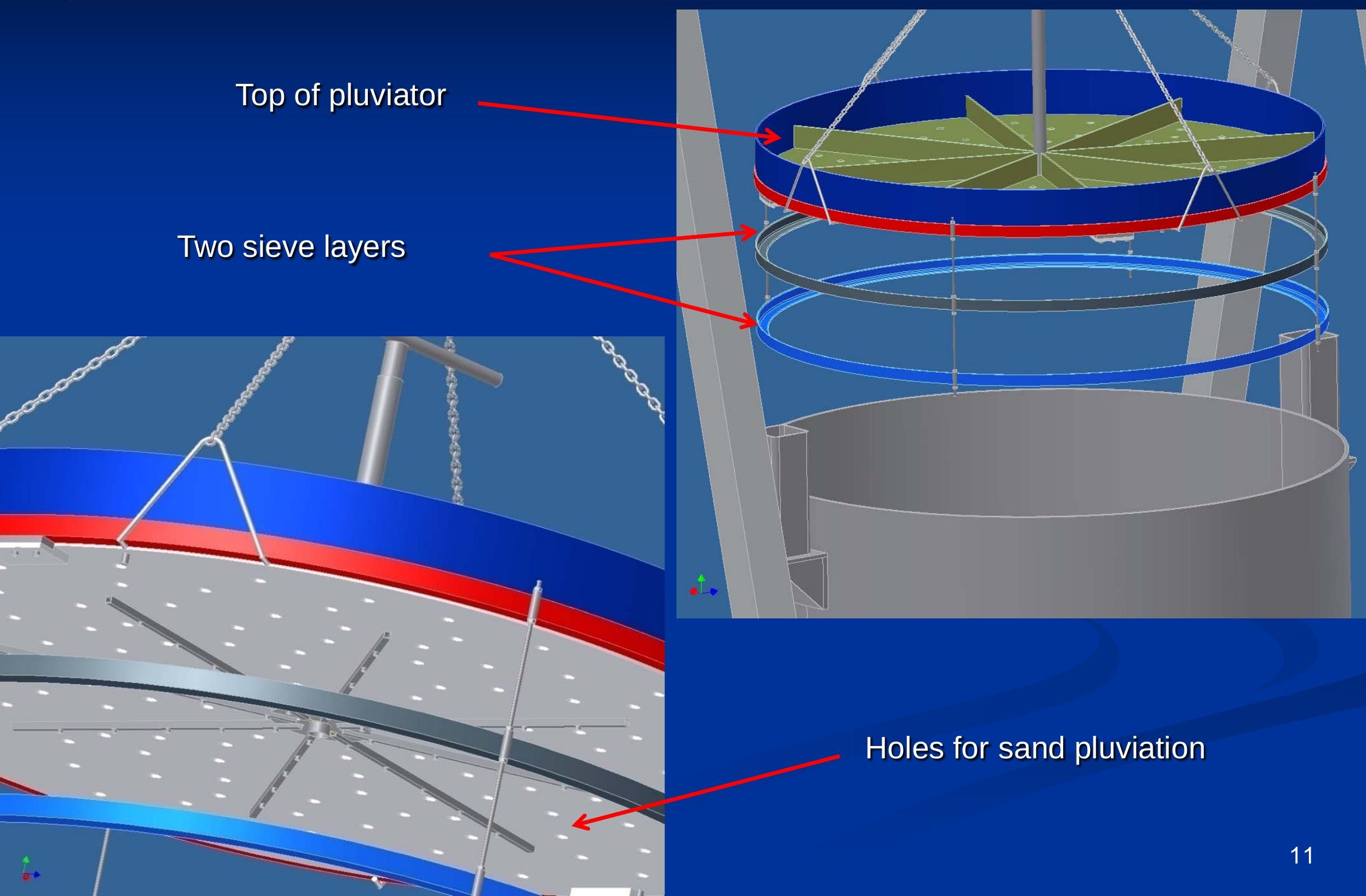

Holes for sand pluviation 


\section{Sand pluviator}

\section{- Schematic view and photo of sand pluviator}
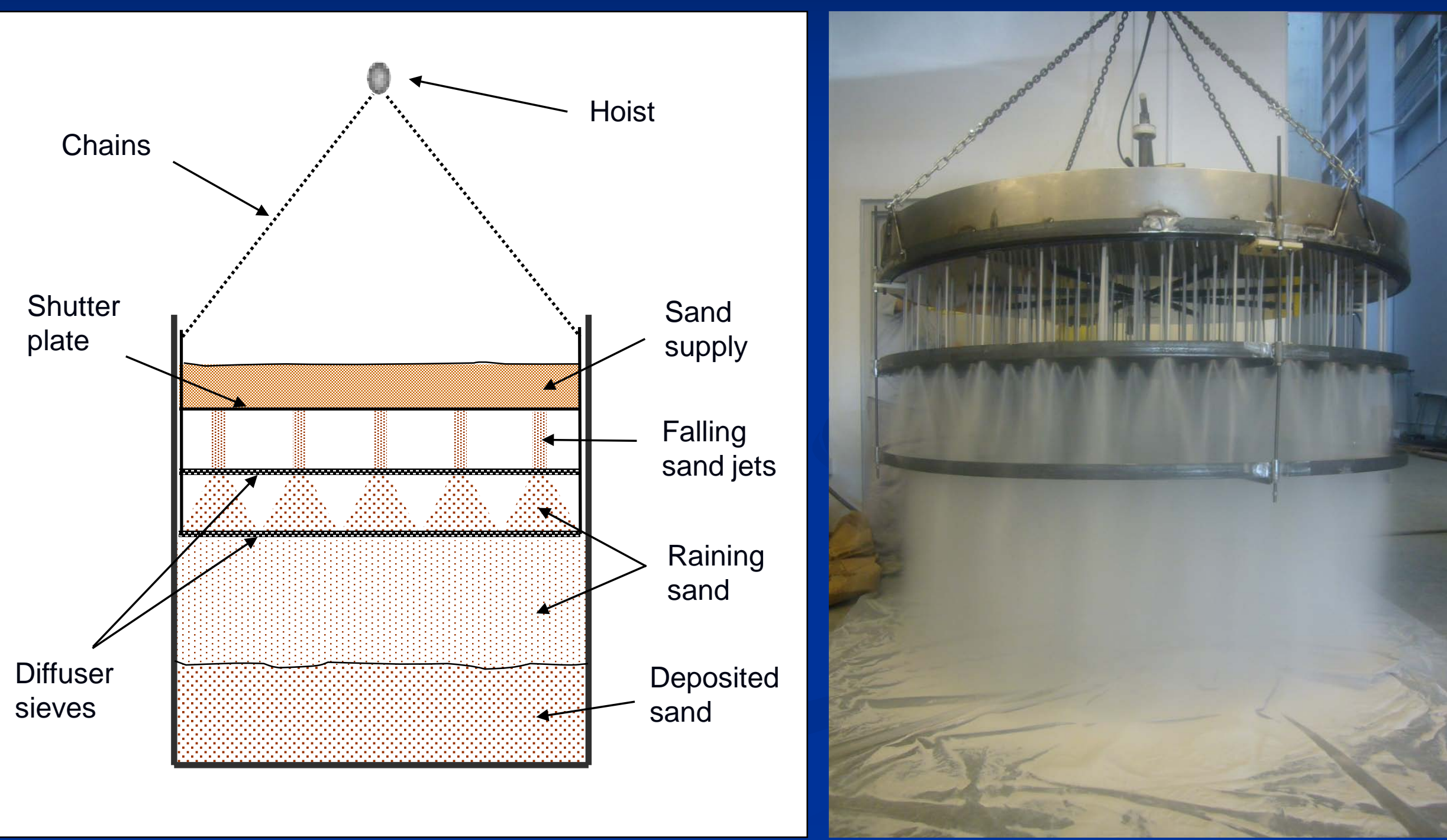


\section{Sample preparation}

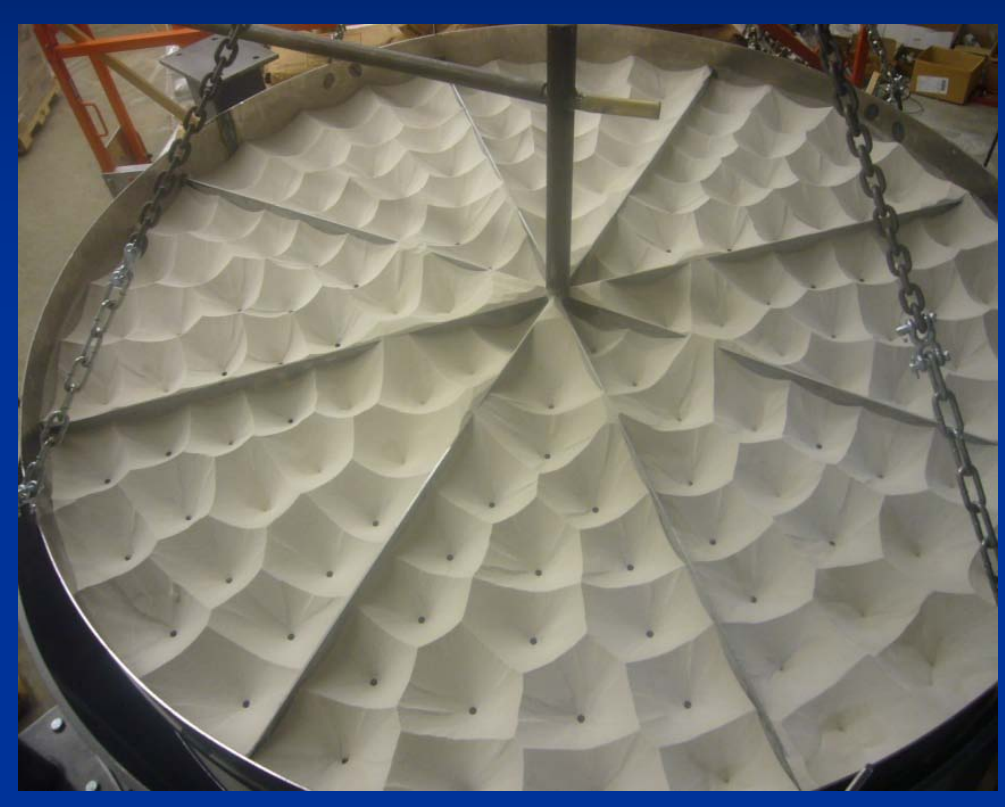

Top view of pluviator

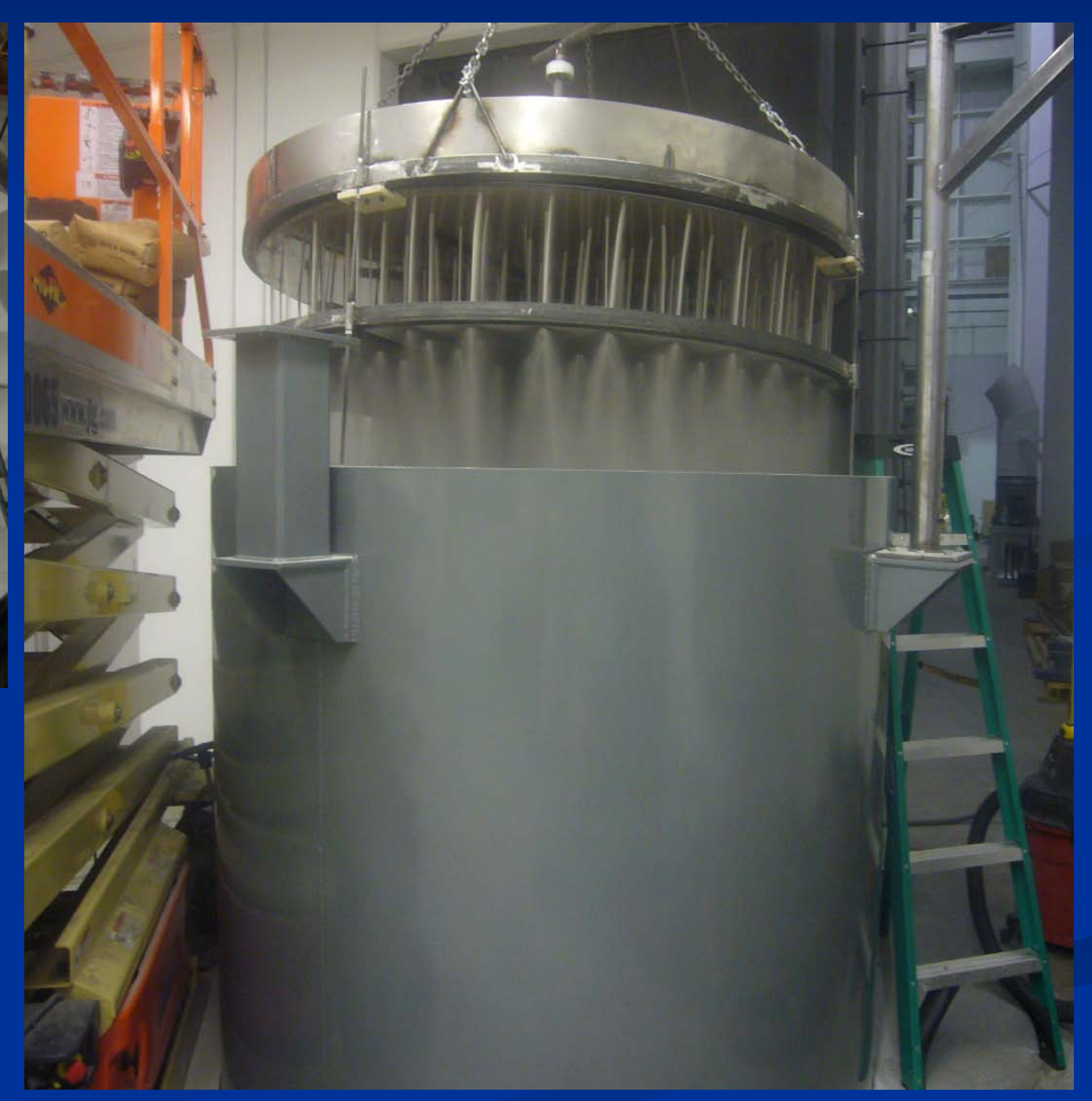

Sand pluviation 


\section{Draining sand}

- Draining sand after the test

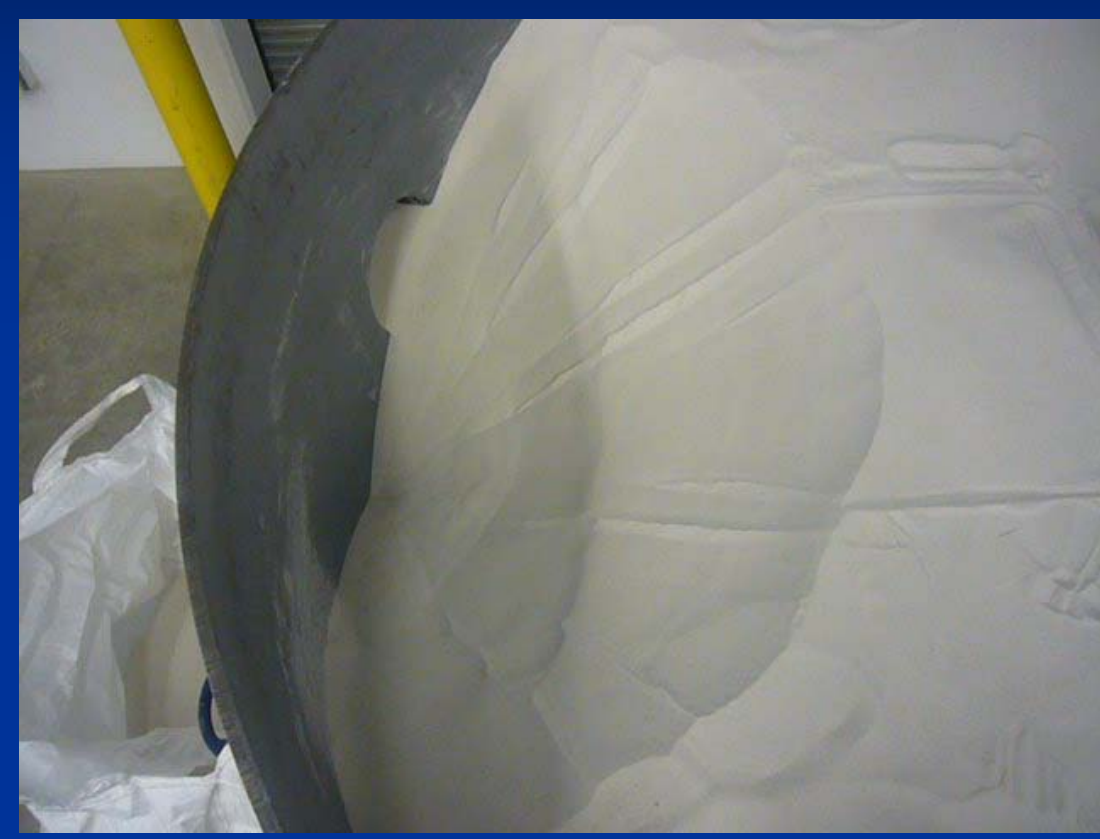




\section{Contents}

- Model pile testing plan

- Model pile testing

- Sample preparation

- Soil tank

- Sand pluviator

- Instrumented model pile

- Driving system

- Preinstallation method

- Jacking system

- Installation of piles for pile group testing

- Lateral loading system

- Comparison of test and analysis results

- Single piles

- Pile groups 


\section{Instrumented model pile}

- Smooth steel pipe ( $D=3 \mathrm{~cm}, \mathrm{~L}=120 \mathrm{~cm}, \mathrm{t}=0.2 \mathrm{~cm})$

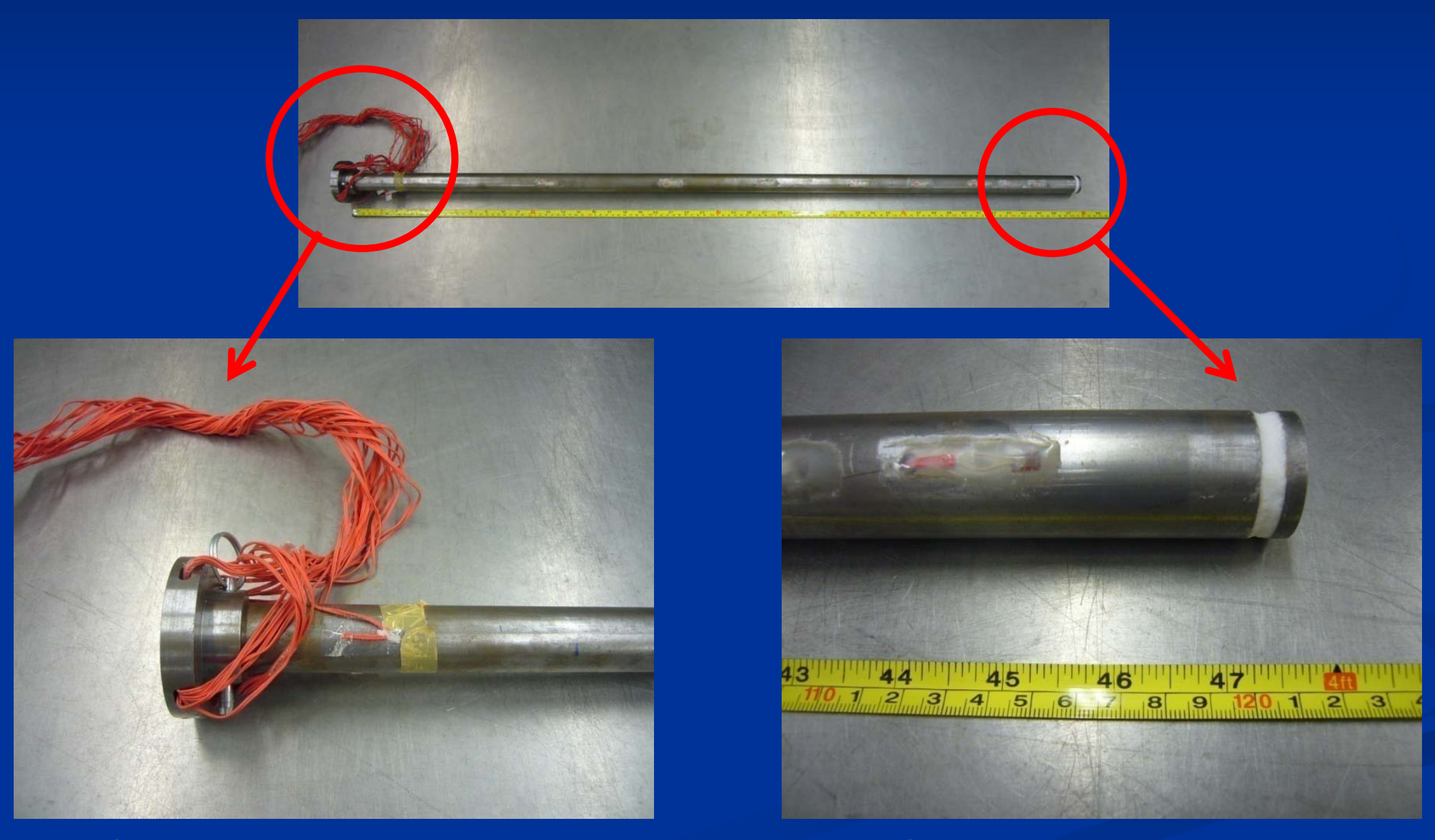

Collected wires at pile head

Closed-ended pile base 


\section{Driving System}

\section{- Installation of driven pile}

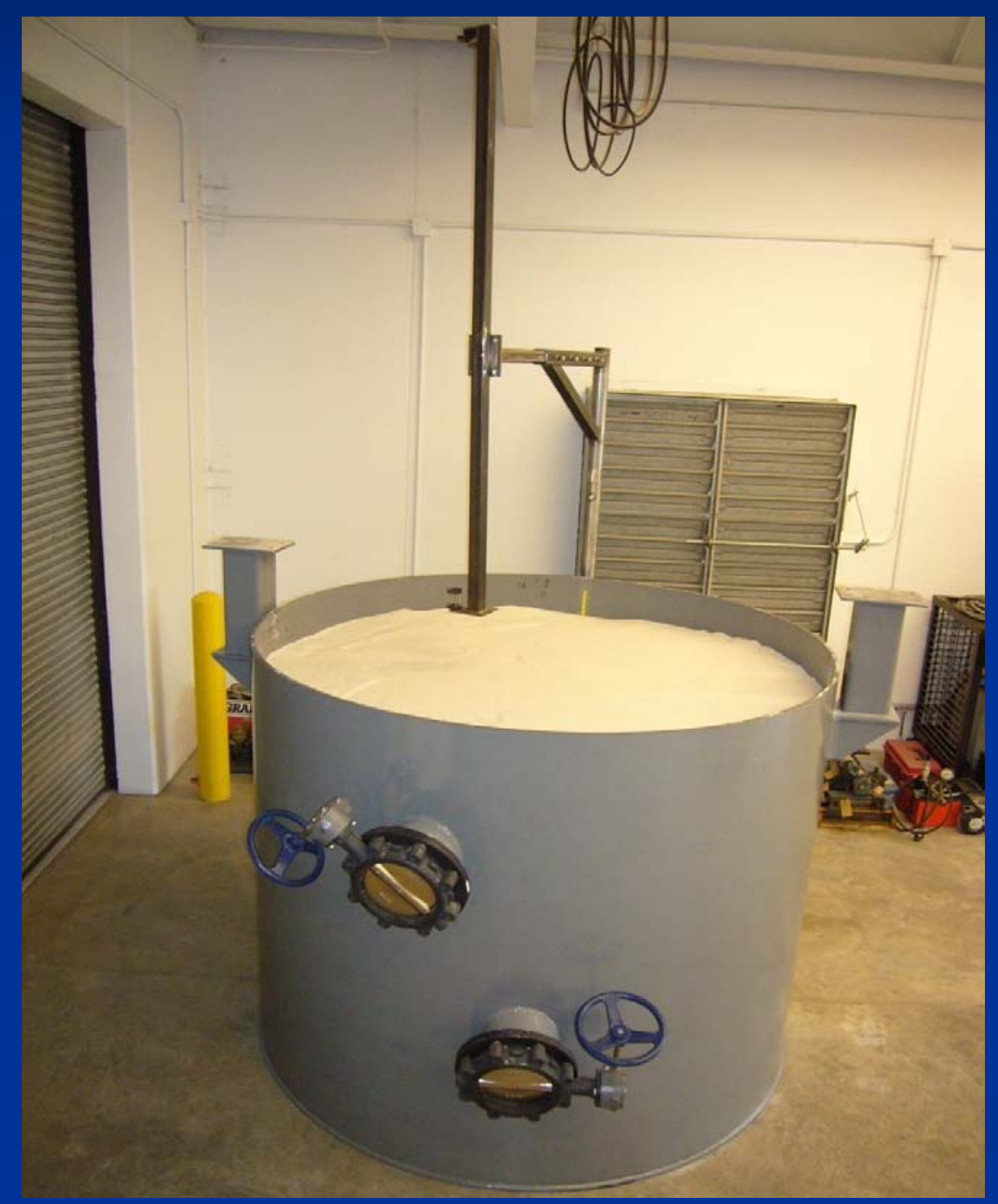

Filled soil tank

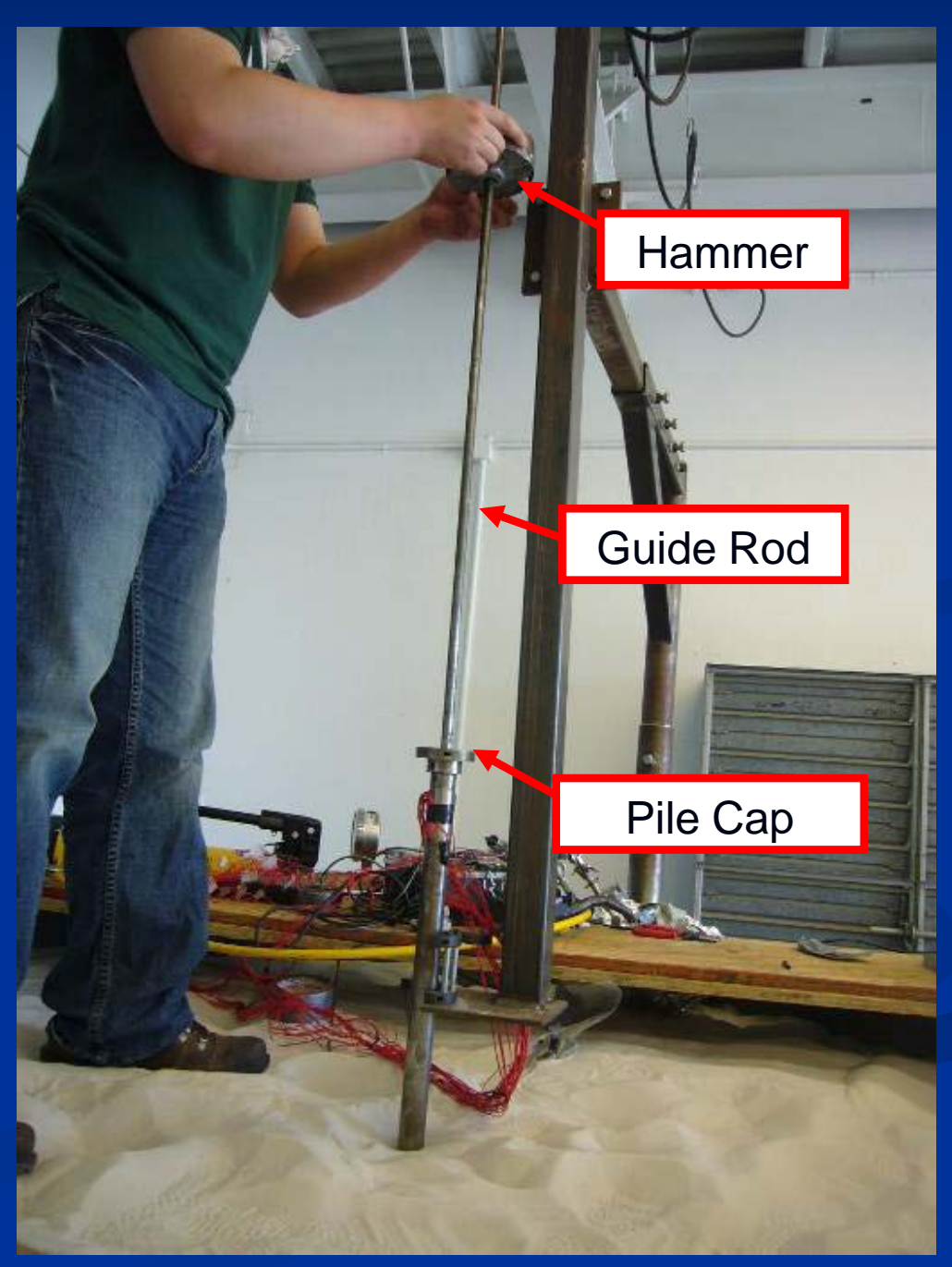




\section{Preinstallation of model pile}
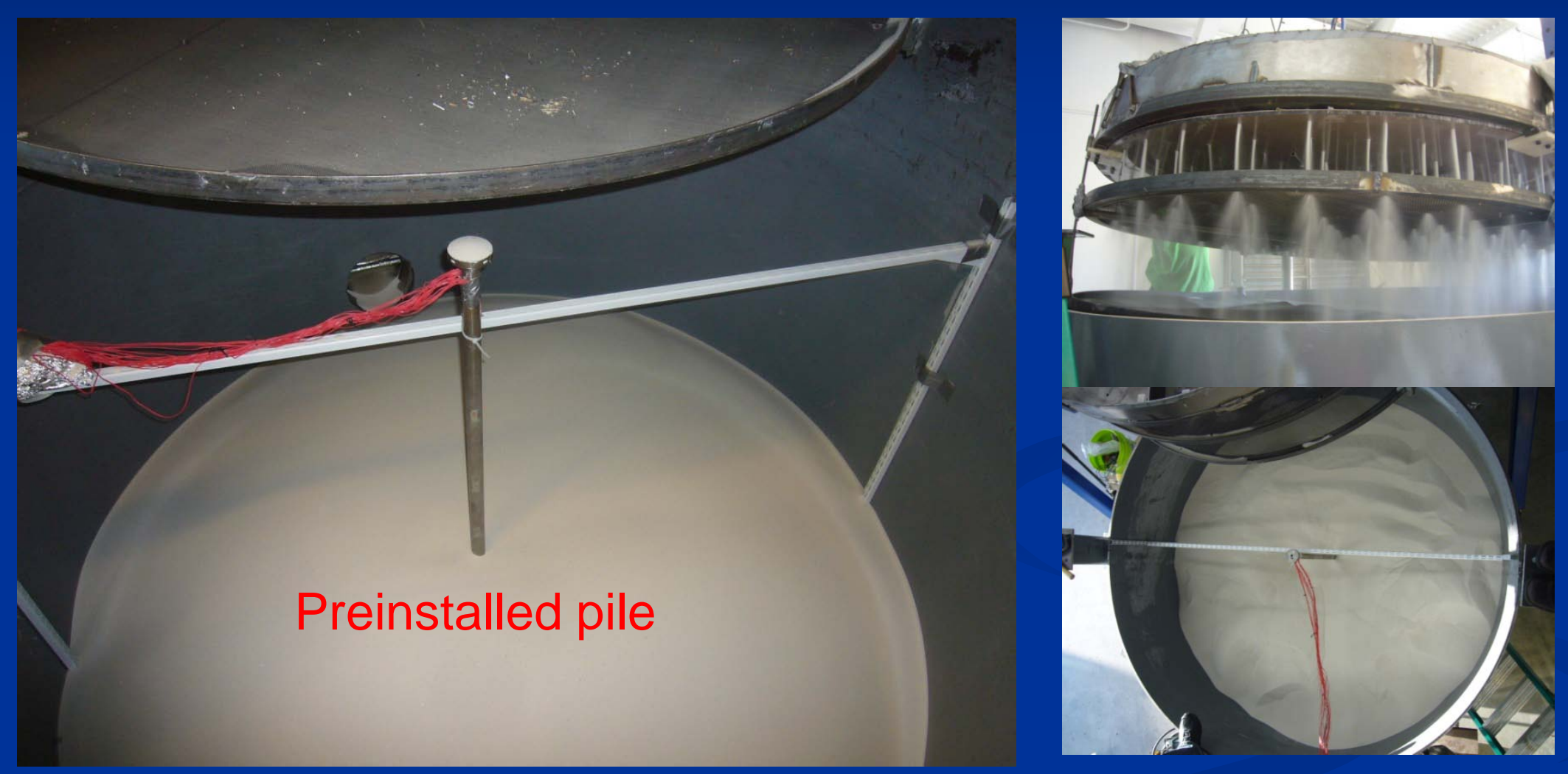


\section{Pile jacking system}
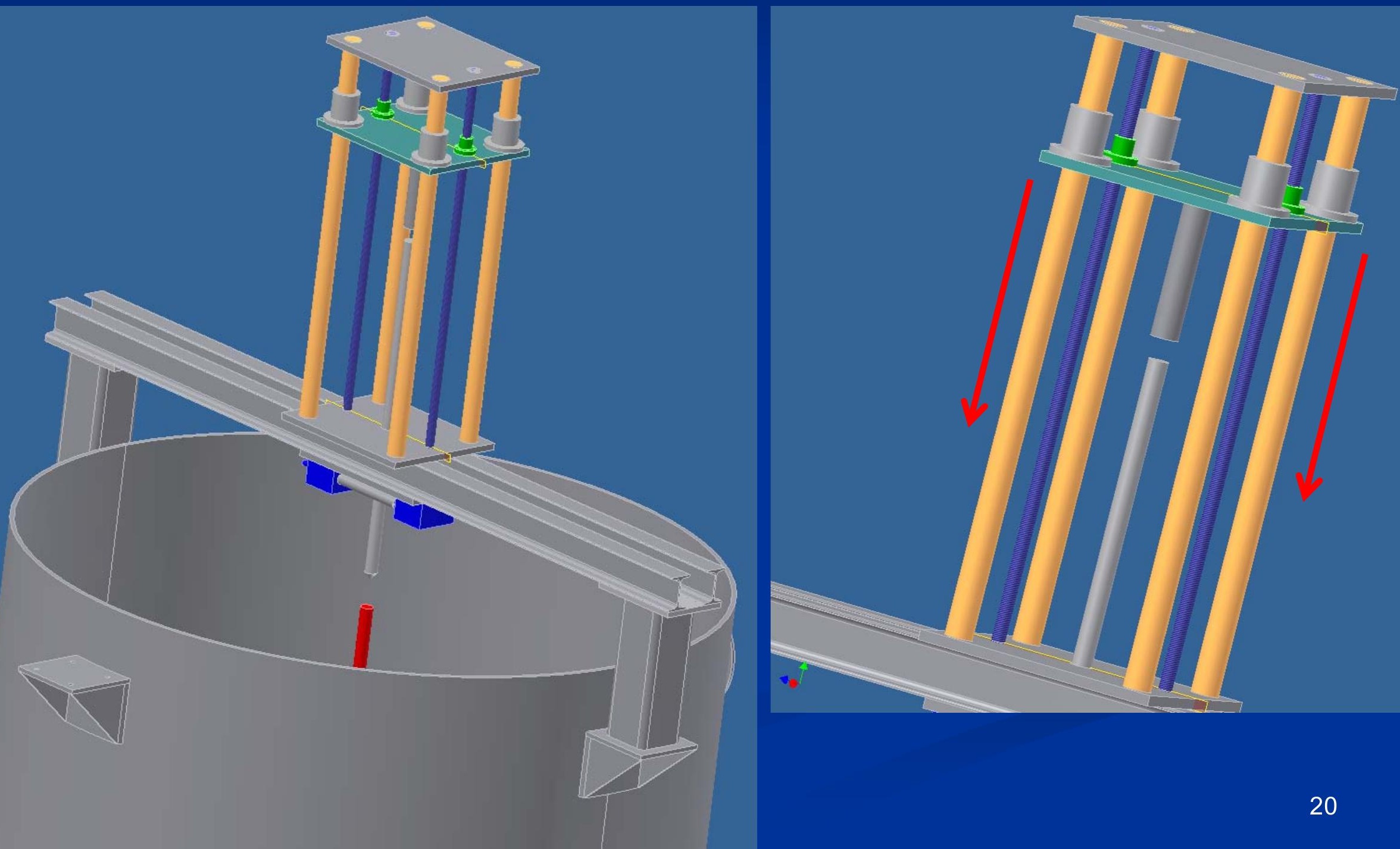


\section{Pile jacking system}

- Max. rate $=5 \mathrm{~cm} / \mathrm{min}$

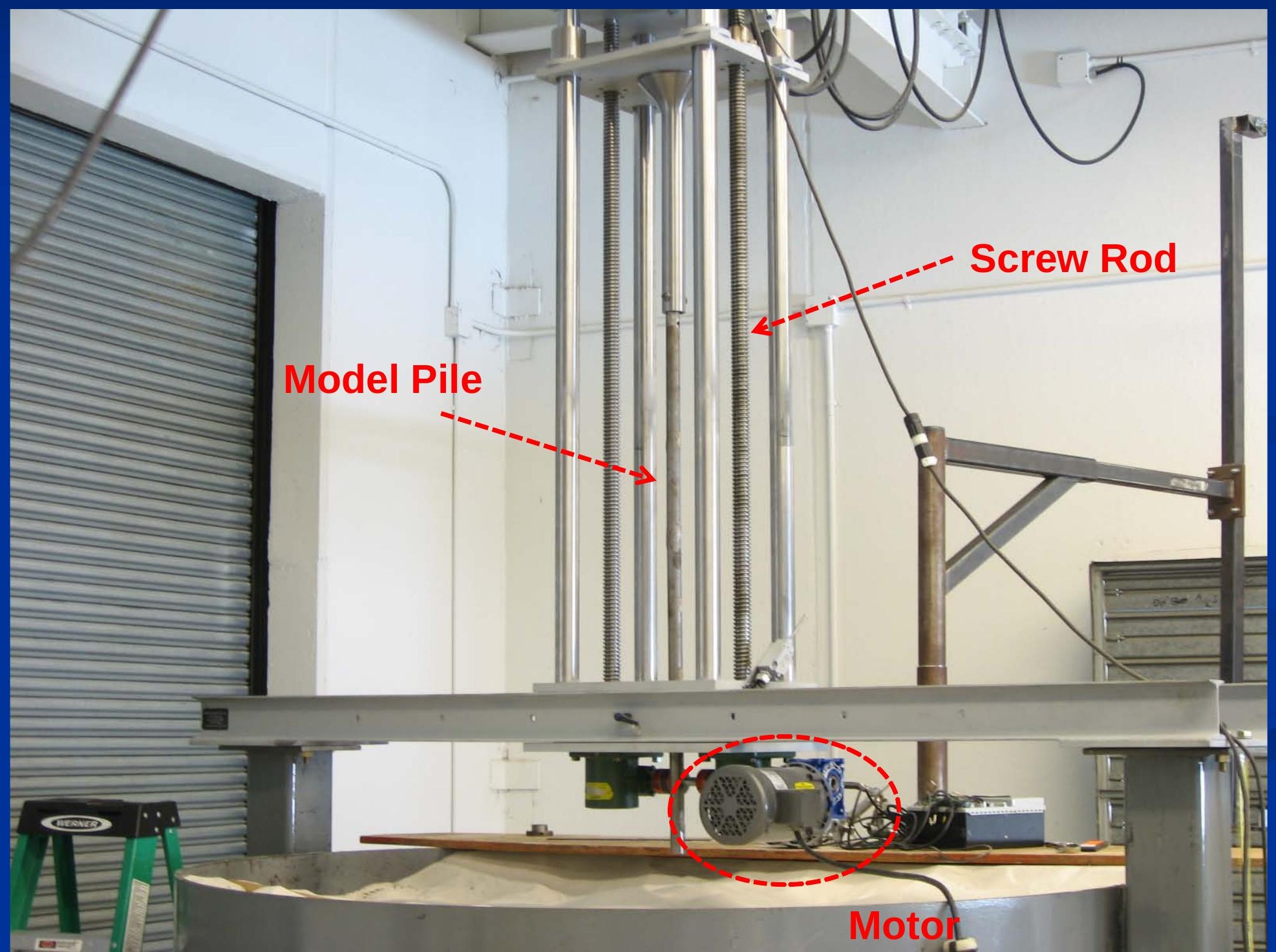




\section{Installation of Group piles}

\section{- Setup}

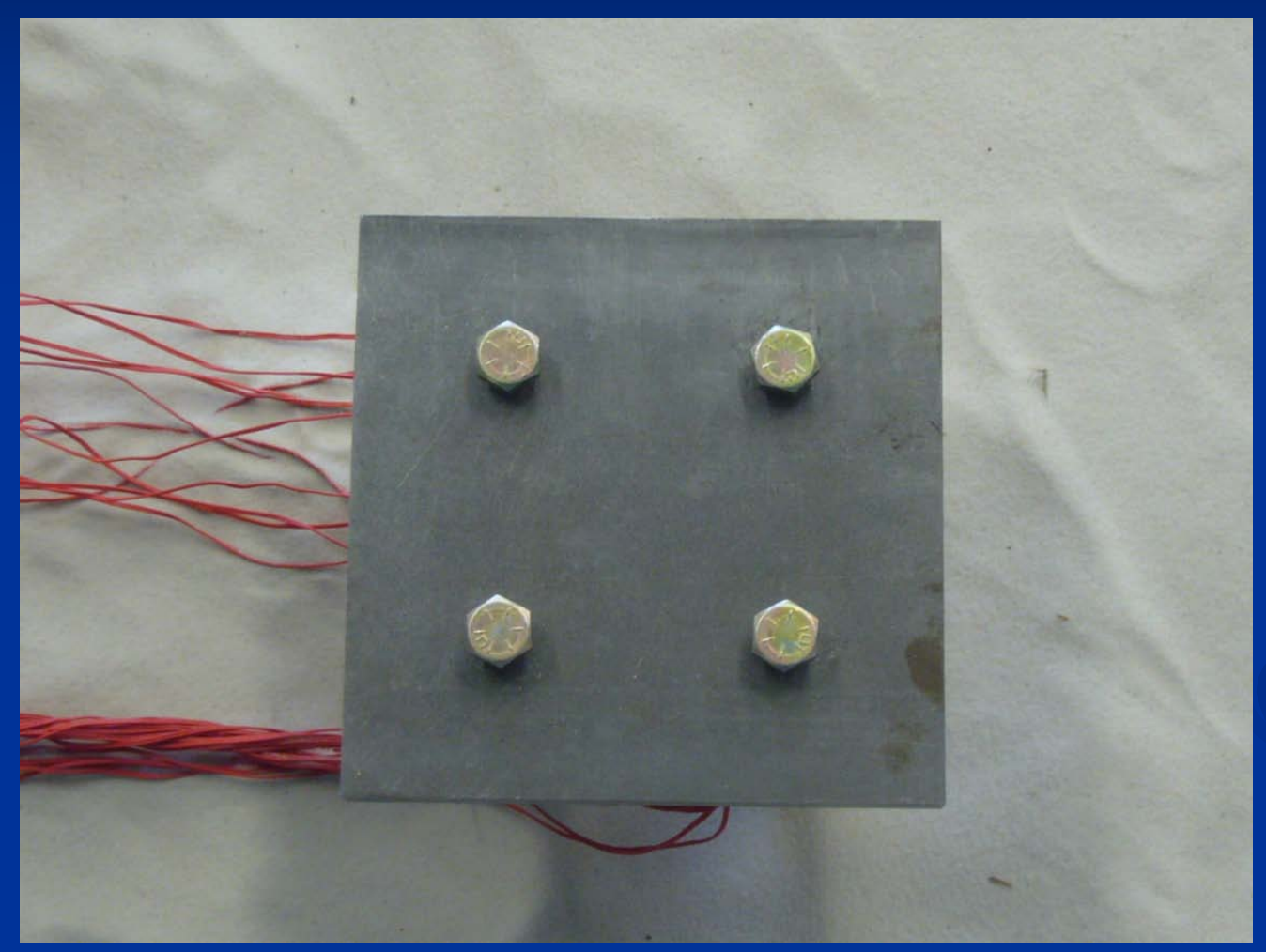

4 Piles \& Steel Cap
Pile driving guide

(Wooden Box Frame)

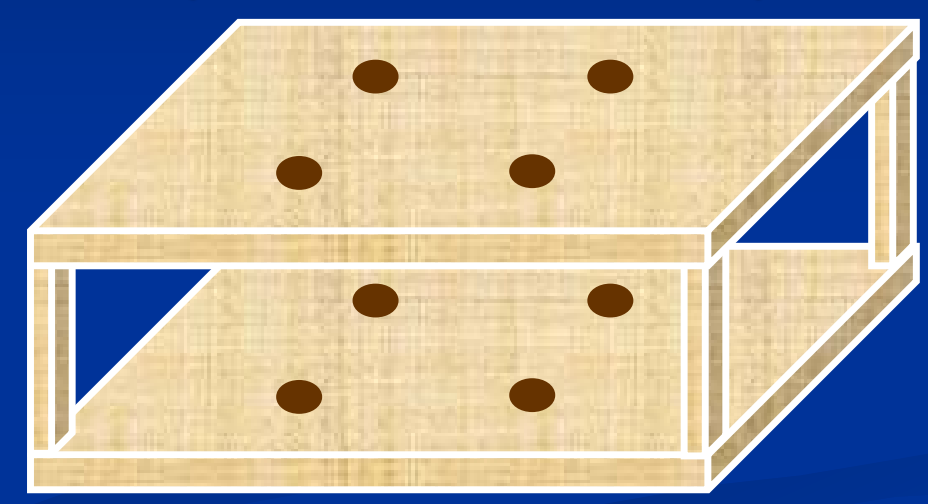

Pile driving order

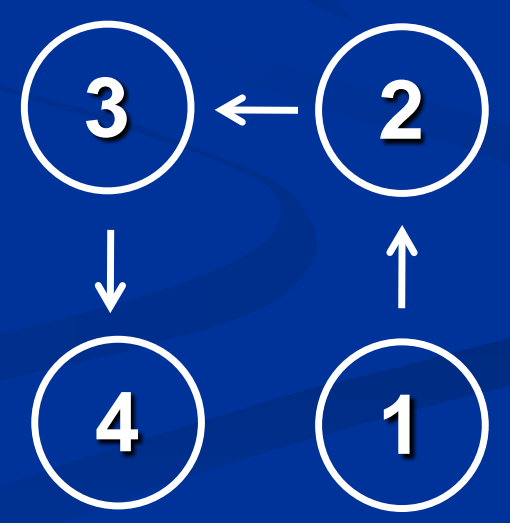




\section{Lateral loading system}

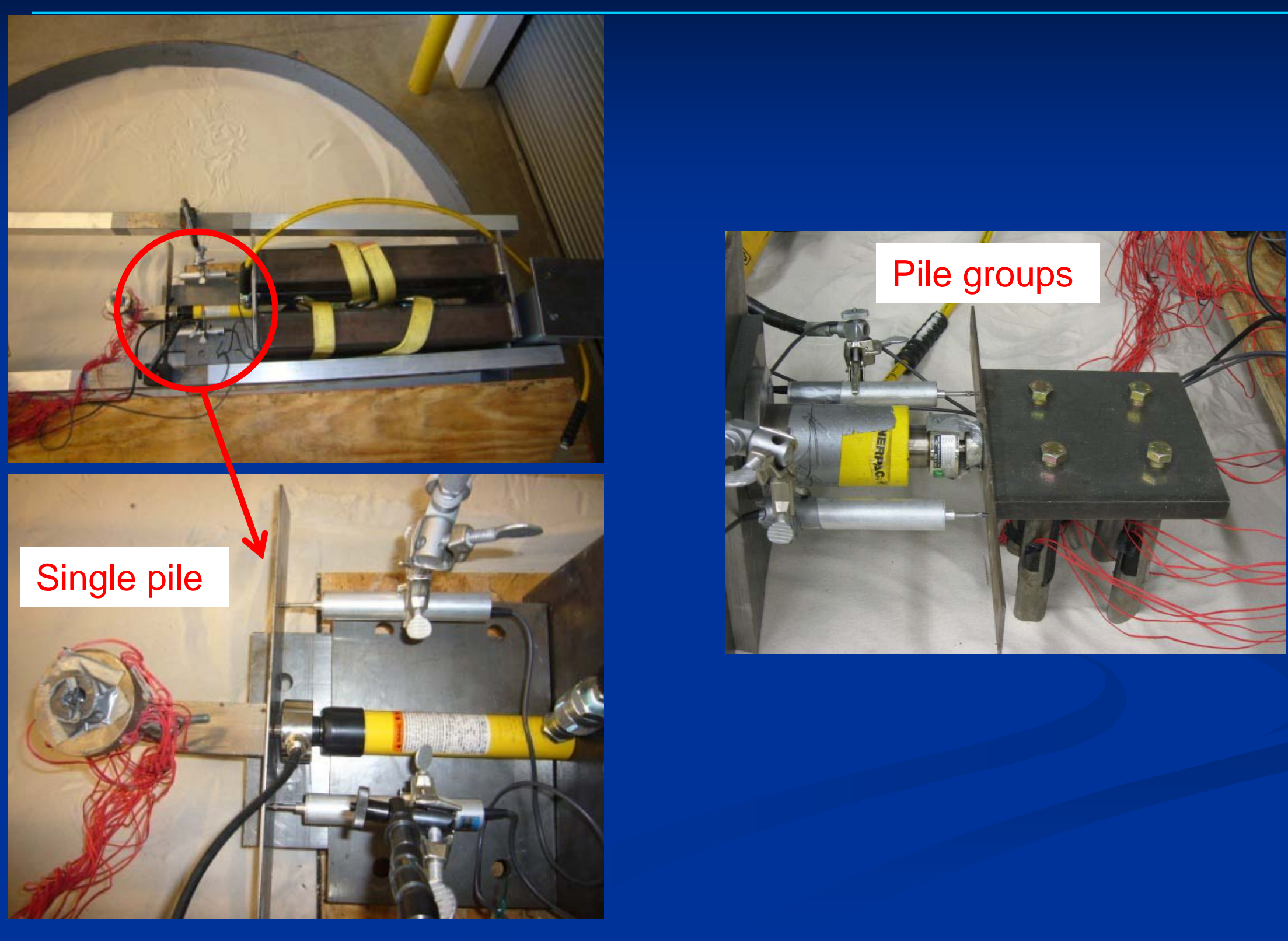




\section{Contents}

- Model pile testing plan

- Model pile testing

- Sample preparation

- Soil tank

- Sand pluviator

- Instrumented model pile

- Driving system

- Preinstallation method

- Jacking system

- Installation of piles for pile group testing

- Lateral loading system

- Comparison of test and analysis results

- Single piles

- Pile groups

- Summary and Conclusions 


\section{Test results: Single pile}

- Lateral load - deflection curves (driven pile)

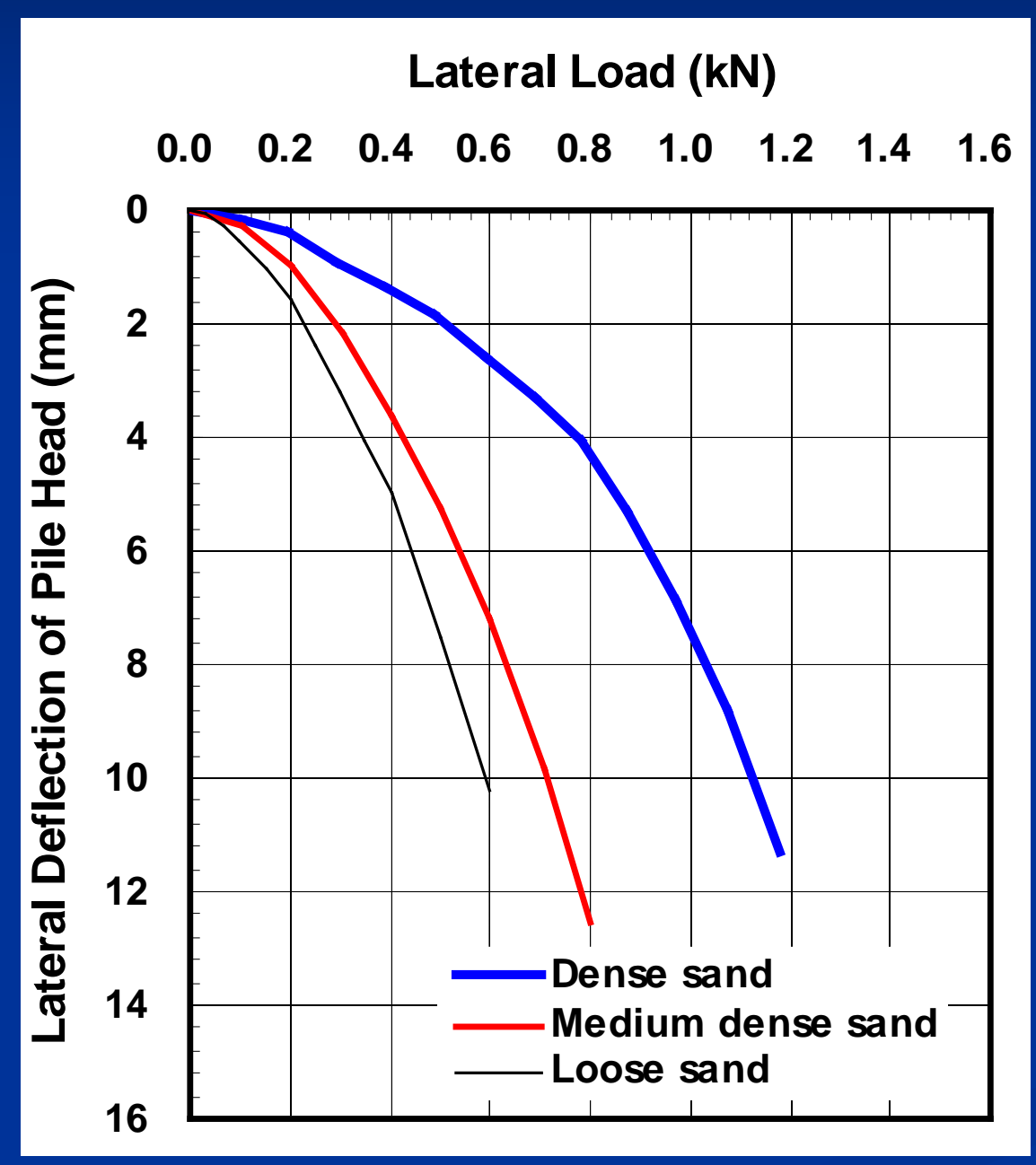

Ultimate lateral loads

$$
\begin{aligned}
& \mathrm{Q}_{\text {lat } 5 \%,} \\
& \mathrm{Q}_{\text {lat }, 10 \%} \\
& \mathrm{Q}_{\text {lat, } 20 \%}
\end{aligned}
$$

corresponding to lateral pile head deflections of 5,10 and $20 \%$ of the pile diameter

\begin{tabular}{|c|c|c|c|}
\hline Sand type & $\begin{array}{c}\mathrm{Q}_{\text {lat, 5\% }} \\
(\mathrm{kN})\end{array}$ & $\begin{array}{c}\mathrm{Q}_{\text {lat, 10\% }} \\
(\mathrm{kN})\end{array}$ & $\begin{array}{c}\mathrm{Q}_{\text {lat, 20\% }} \\
(\mathrm{kN})\end{array}$ \\
\hline Dense & 0.42 & 0.65 & 0.92 \\
\hline Medium dense & 0.24 & 0.36 & 0.54 \\
\hline Loose & 0.19 & 0.29 & 0.44 \\
\hline
\end{tabular}




\section{Test results: Single pile}

- Bending moment profiles (driven pile)

Dense sand $\left(D_{R}=91 \%\right)$

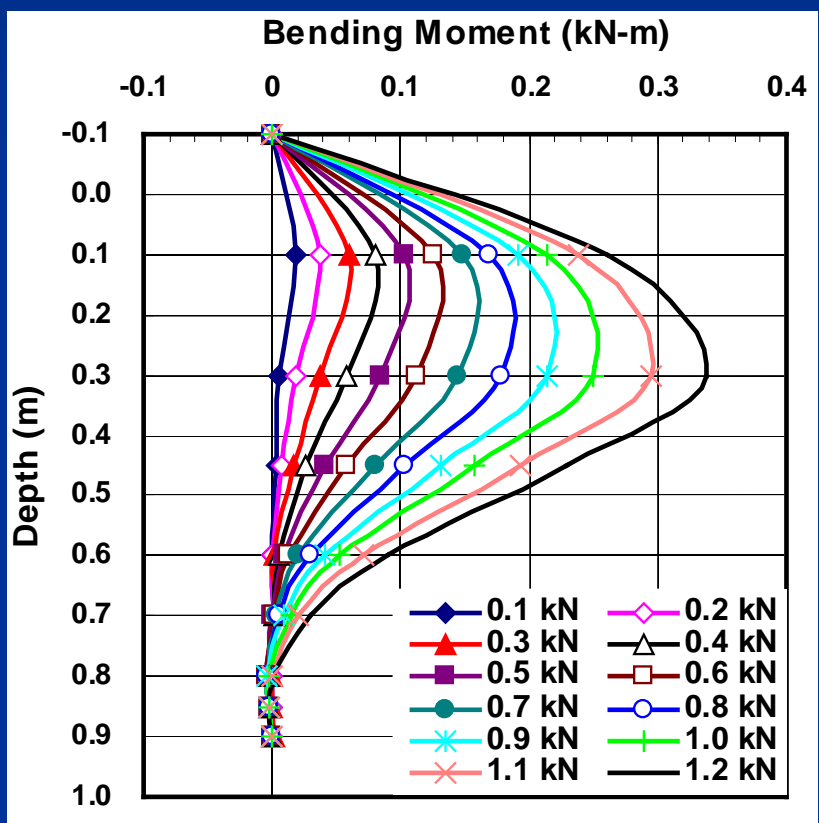

Bending moment

$$
M=\frac{E I \cdot\left(\varepsilon_{T}-\varepsilon_{c}\right)}{h}
$$

Rollins et al. (1998)
Medium dense sand $\left(D_{R}=59 \%\right)$

Loose sand $\left(D_{R}=38 \%\right)$
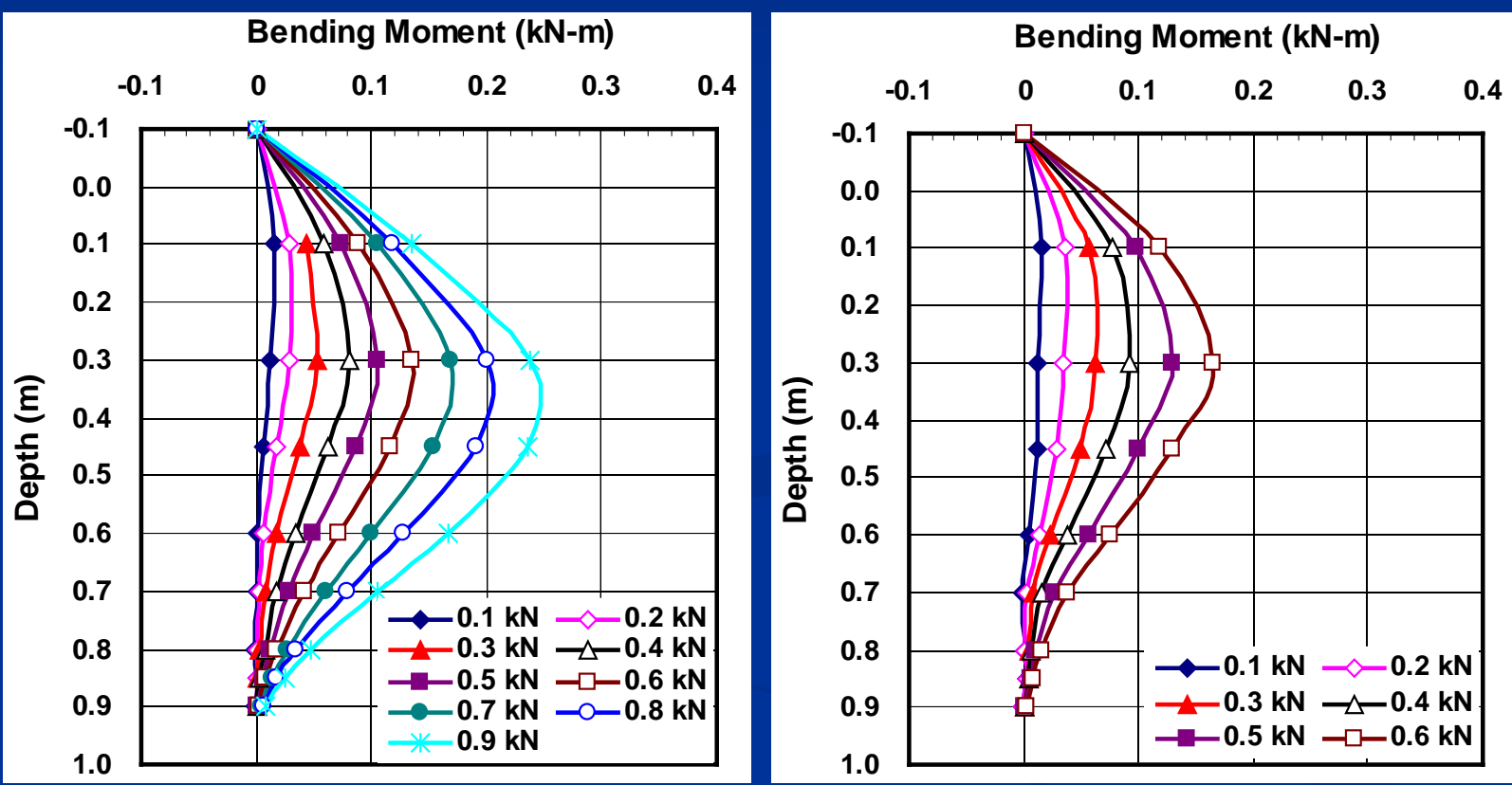

$\varepsilon_{T}$ : tensile strain

$\varepsilon_{C}$ : compressive strain

$h$ : horizontal distance between gauges

$E I$ : bending stiffness of pile 


\section{Test results: Single pile}

- Lateral load - deflection curves (jacked pile)

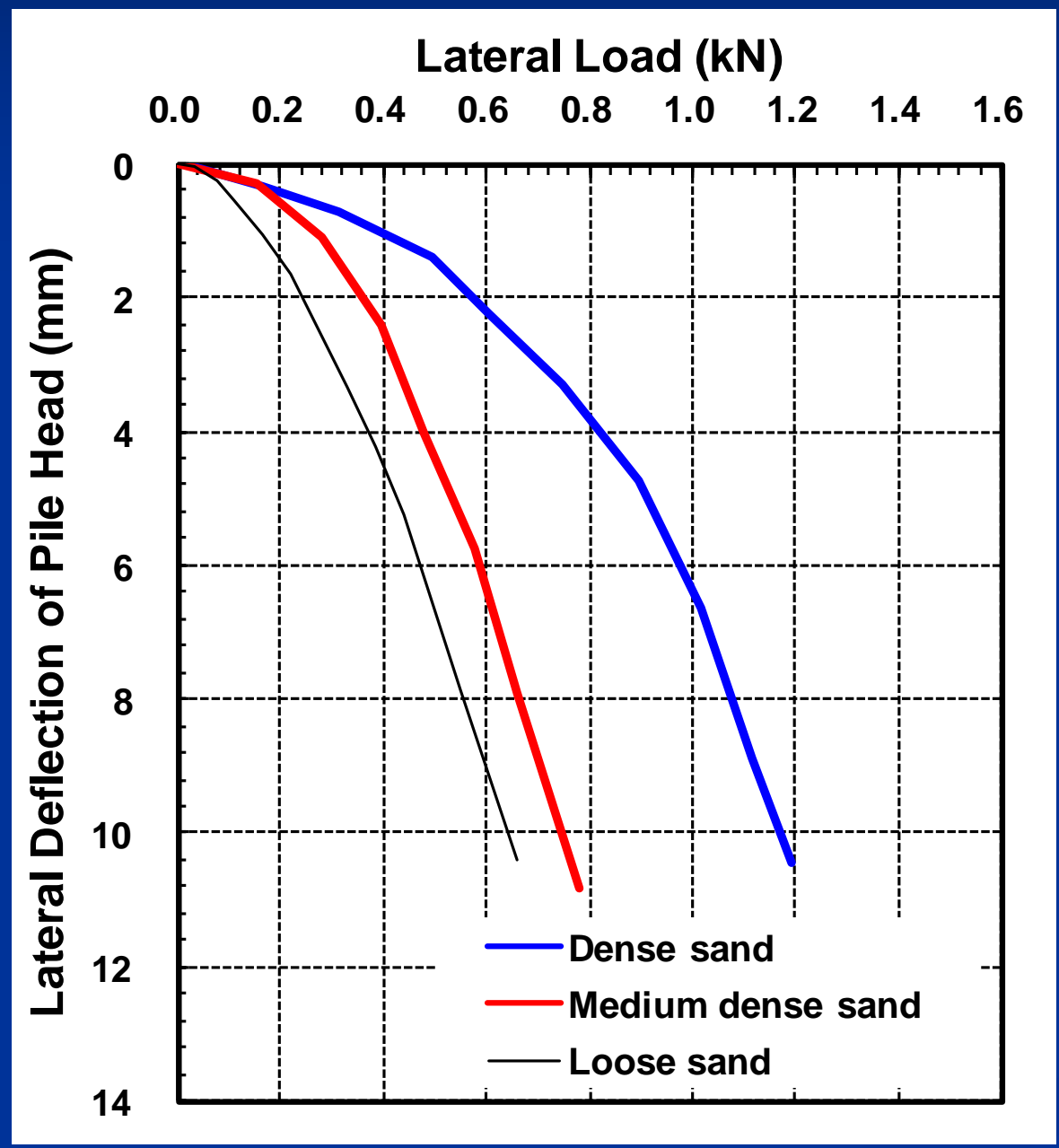

\begin{tabular}{|c|c|c|c|}
\hline Sand type & $\begin{array}{c}\mathrm{Q}_{\text {lat, 5\% }} \\
(\mathrm{kN})\end{array}$ & $\begin{array}{c}\mathrm{Q}_{\text {lat, 10\% }} \\
(\mathrm{kN})\end{array}$ & $\begin{array}{c}\mathrm{Q}_{\text {lat, 20\% }} \\
(\mathrm{kN})\end{array}$ \\
\hline Dense & 0.51 & 0.71 & 0.97 \\
\hline Medium dense & 0.32 & 0.42 & 0.59 \\
\hline Loose & 0.21 & 0.31 & 0.47 \\
\hline
\end{tabular}




\section{Test results: Single pile}

- Bending moment profiles (jacked pile)

Dense sand $\left(D_{R}=91 \%\right)$

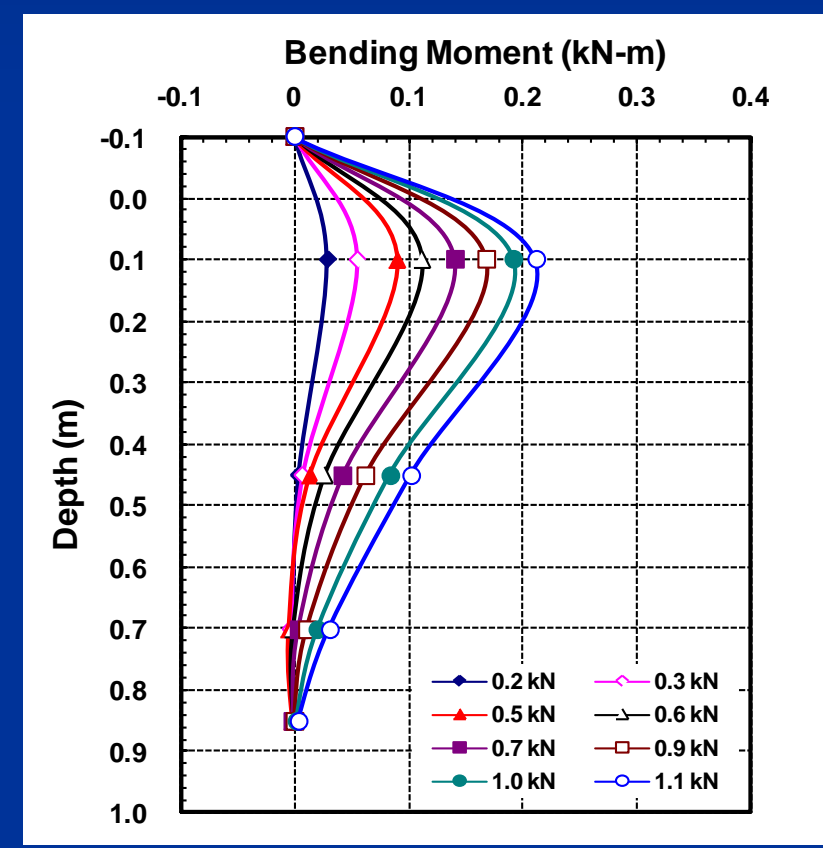

Medium dense sand $\left(D_{R}=59 \%\right)$

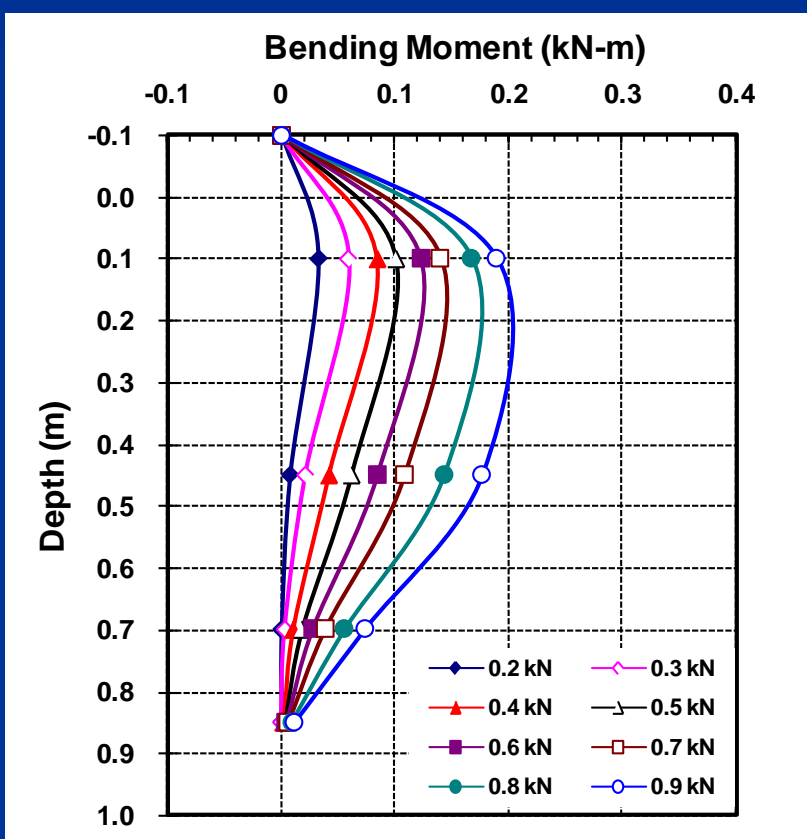

Loose sand $\left(D_{R}=38 \%\right)$

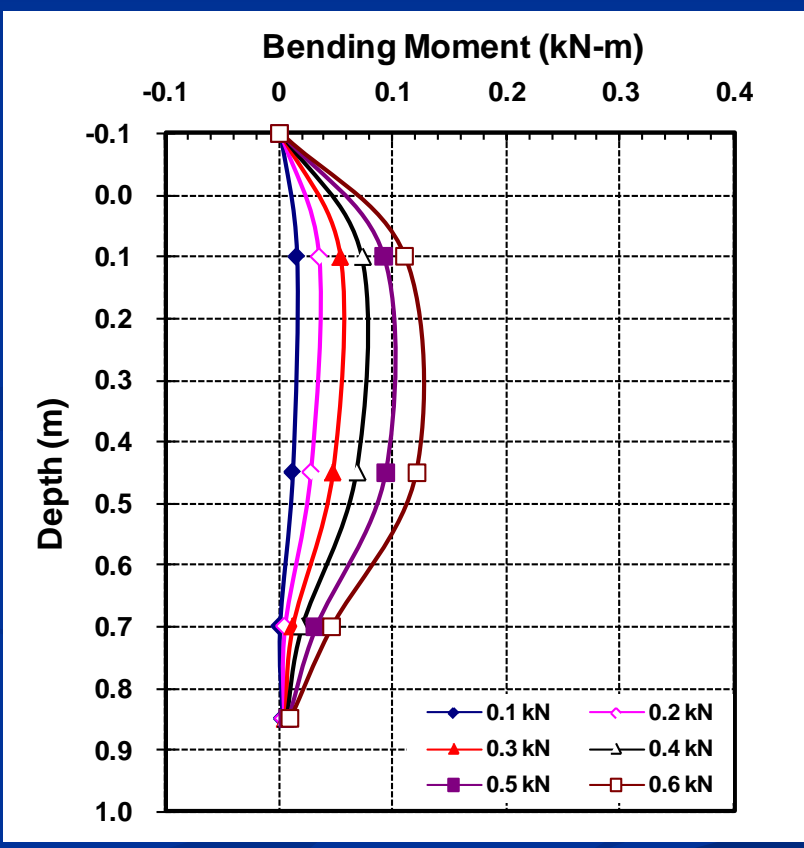

$1.0 \mathrm{kN}-\mathrm{m}=0.738 \mathrm{lb}-\mathrm{kips}$ 


\section{Test results: Single pile}

- Lateral load - deflection curves (preinstalled pile)

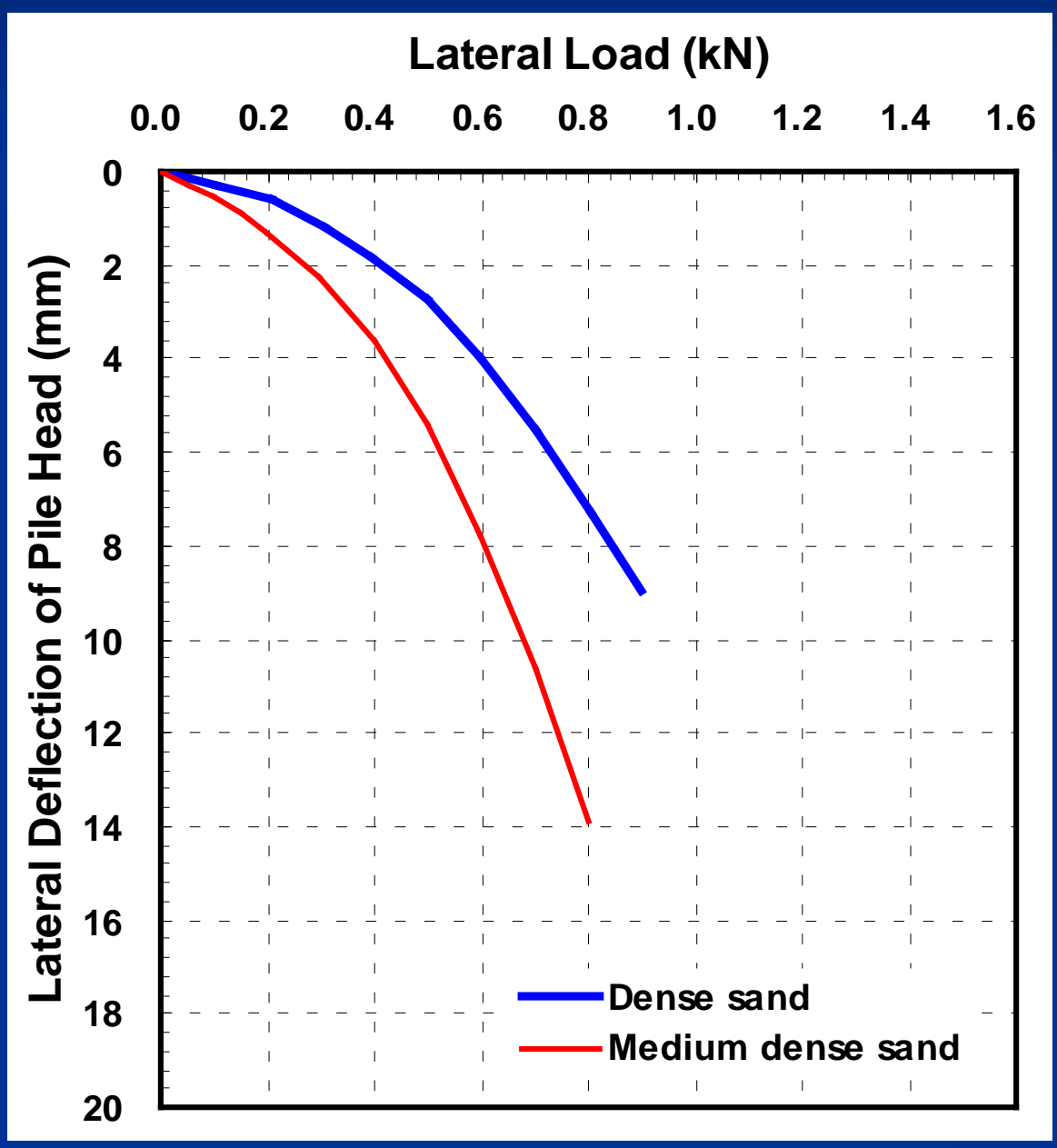

\begin{tabular}{|c|c|c|c|}
\hline Sand type & $\begin{array}{c}\mathrm{Q}_{\text {lat, 5\% }} \\
(\mathrm{kN})\end{array}$ & $\begin{array}{c}\mathrm{Q}_{\text {lat, 10\% }} \\
(\mathrm{kN})\end{array}$ & $\begin{array}{c}\mathrm{Q}_{\text {lat, 20\% }} \\
(\mathrm{kN})\end{array}$ \\
\hline Dense & 0.35 & 0.52 & 0.73 \\
\hline Medium dense & 0.22 & 0.35 & 0.52 \\
\hline
\end{tabular}




\section{Test results: Single pile}

- Bending moment profiles (preinstalled pile)

$$
\text { Dense sand }\left(D_{R}=91 \%\right)
$$

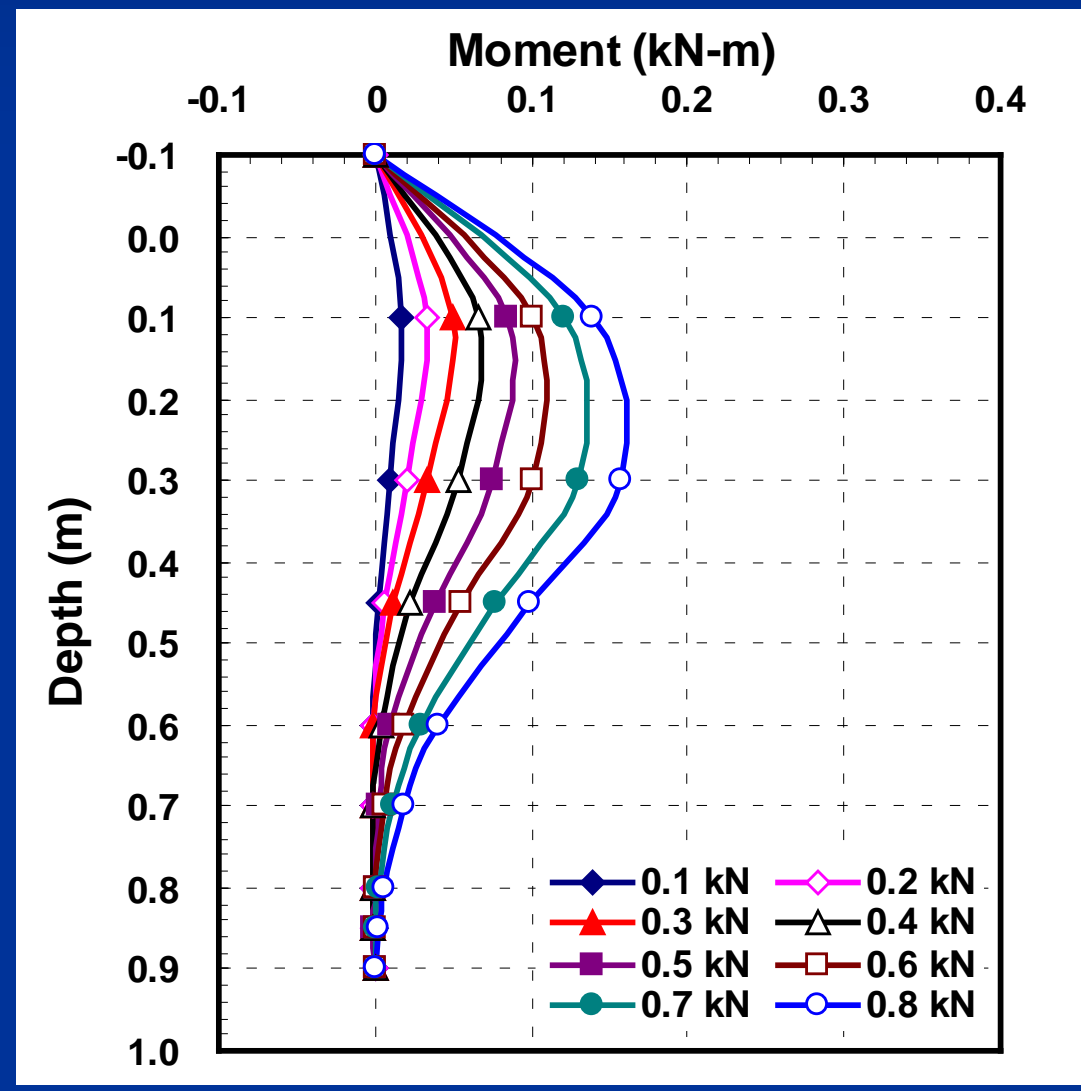

Medium dense sand $\left(D_{R}=59 \%\right)$

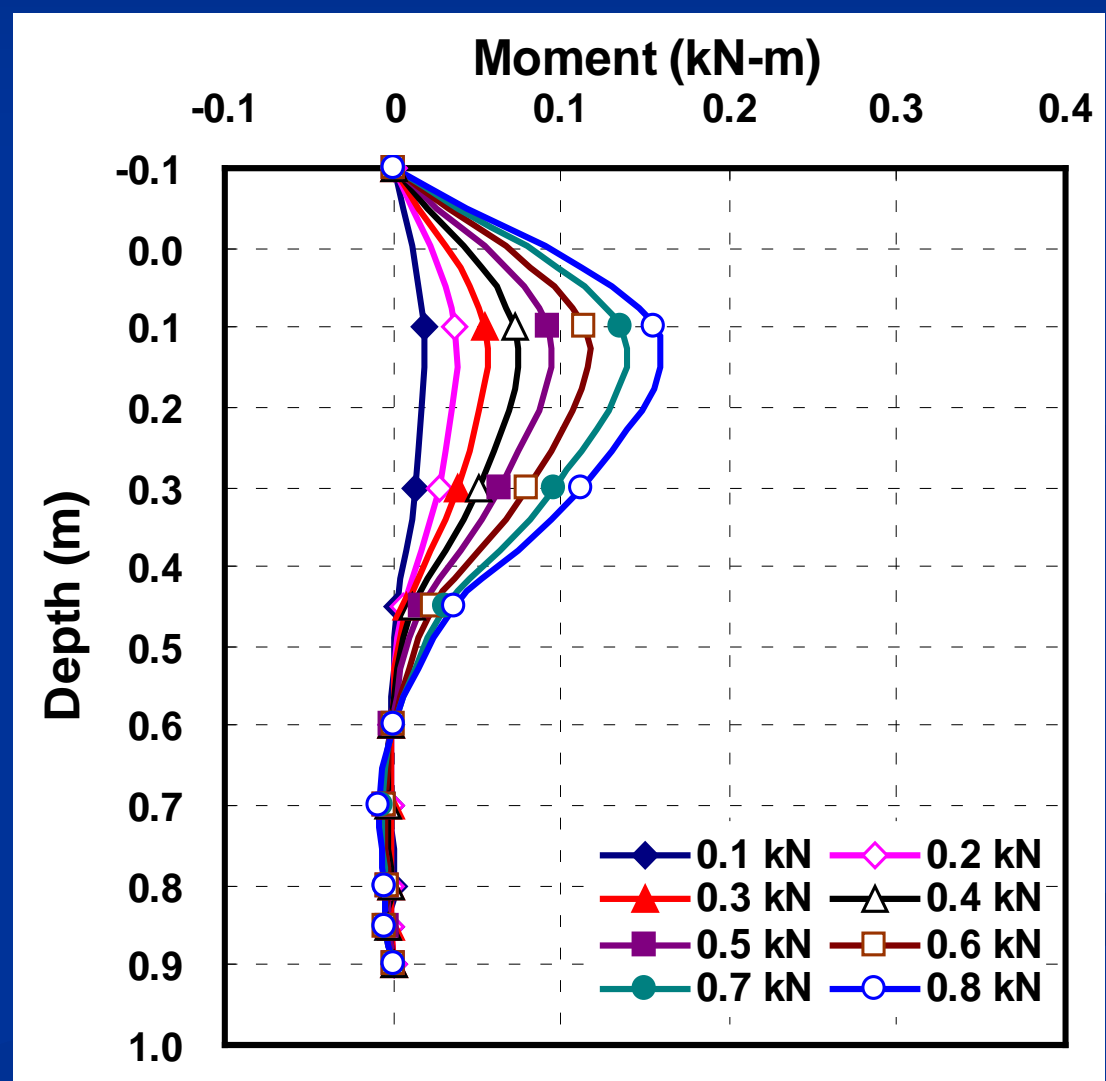




\section{Test results: Single pile}

- Load-deflection curves and bending moment profiles (Multij-layer soils)
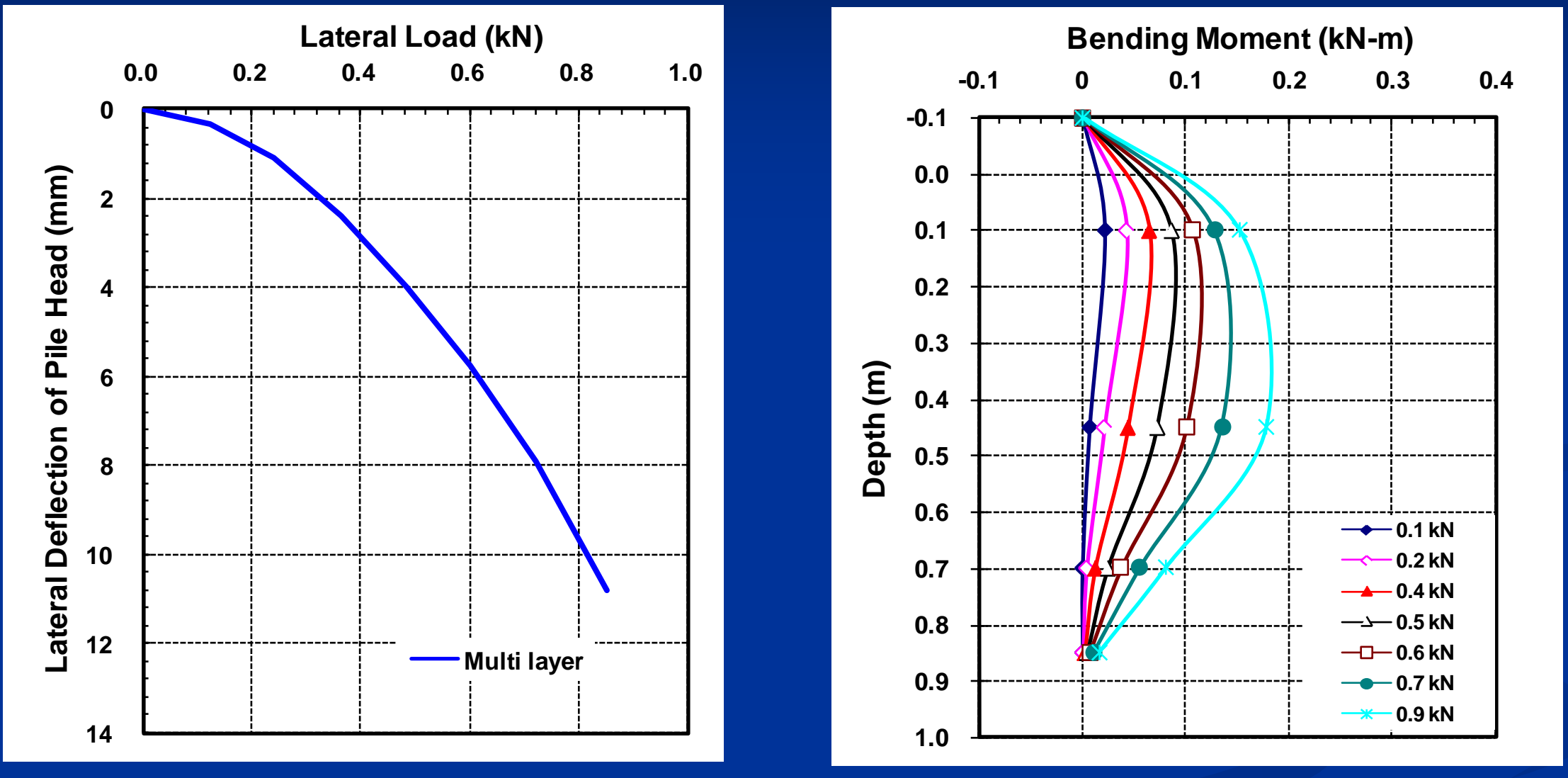

\begin{tabular}{|c|c|c|c|}
\hline Sand type & $\begin{array}{c}\mathrm{Q}_{\text {lat, 5\% }} \\
(\mathrm{kN})\end{array}$ & $\begin{array}{c}\mathrm{Q}_{\text {lat, 10\% }} \\
(\mathrm{kN})\end{array}$ & $\begin{array}{c}\mathrm{Q}_{\text {lat, 20\% }} \\
(\mathrm{kN})\end{array}$ \\
\hline Multi layer & 0.28 & 0.41 & 0.61 \\
\hline
\end{tabular}

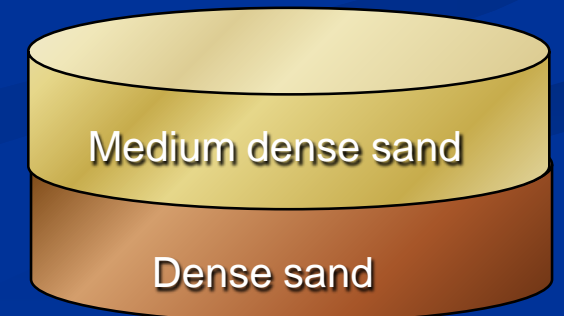

Soil Tank Soil Tank 


\section{Effect of pile installation method}

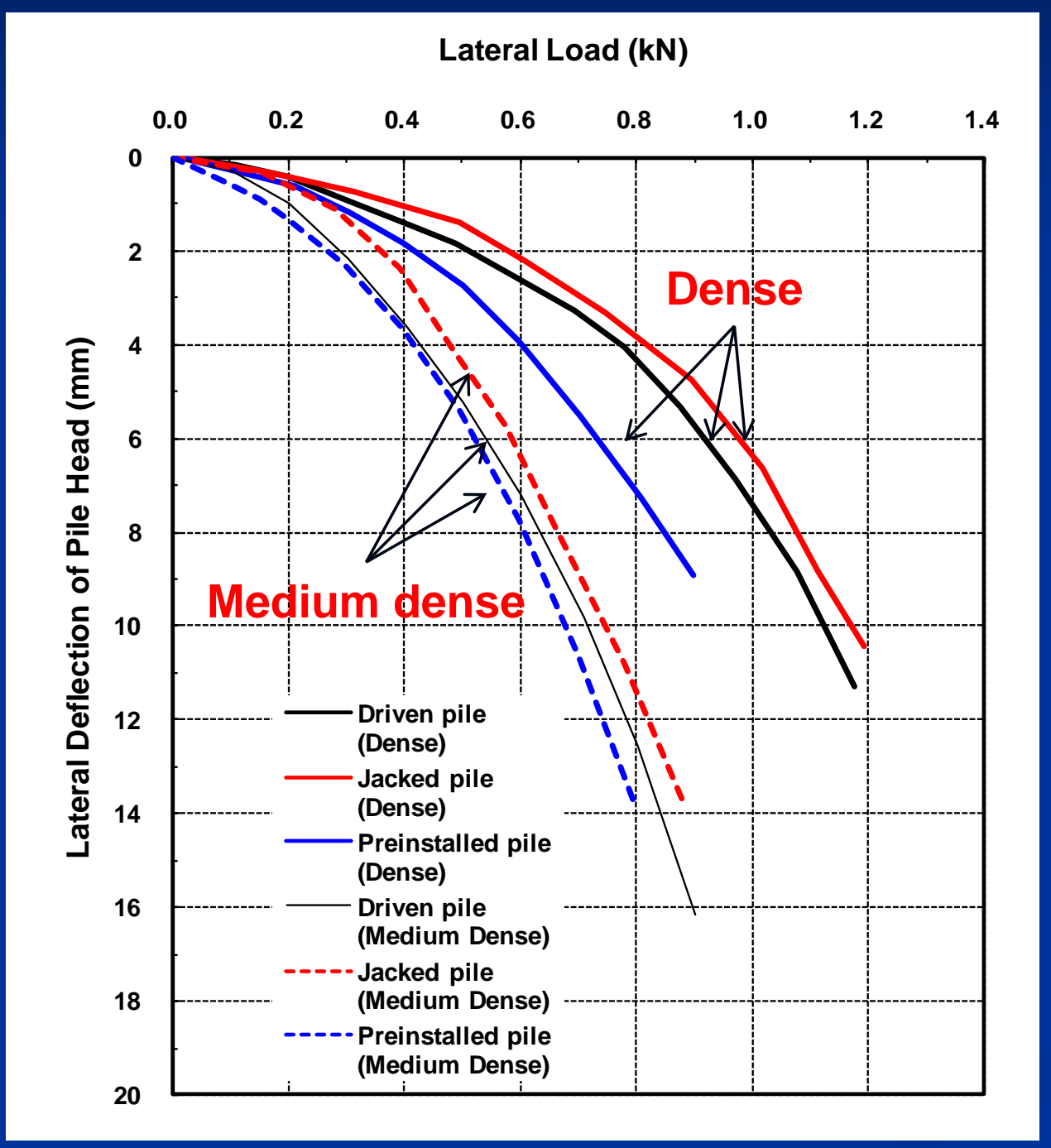

\section{Dense sand}

$\mathrm{Q}_{\text {lat,10\% }}$ (preinstalled piles)

$=73 \%$ of $\mathrm{Q}_{\text {lat }, 10 \%}$ (jacked piles)

\begin{tabular}{|c|c|}
\hline $\begin{array}{c}\text { Installation } \\
\text { Method }\end{array}$ & $\begin{array}{c}\mathrm{Q}_{\text {lat, 10\% }} \\
(\mathrm{kN})\end{array}$ \\
\hline Jacked & 0.71 \\
\hline Driven & 0.65 \\
\hline Preinstalled & 0.52 \\
\hline
\end{tabular}

Medium dense sand

$\mathrm{Q}_{\text {lat, } 10 \%}$ (preinstalled piles)

$=84 \%$ of $\mathrm{Q}_{\text {lat }, 10 \%}$ (jacked piles)

\begin{tabular}{|c|c|}
\hline $\begin{array}{c}\text { Installation } \\
\text { Method }\end{array}$ & $\begin{array}{c}\mathrm{Q}_{\text {lat, 10\% }} \\
(\mathrm{kN})\end{array}$ \\
\hline Jacked & 0.42 \\
\hline Driven & 0.36 \\
\hline Preinstalled & 0.35 \\
\hline
\end{tabular}




\section{Comparison of Result with Predictions}

- Comparison of predictions (PYGMY, present analysis) with measurements from model piles driven in dense, medium dense, and loose sand

Dense sand $\left(D_{R}=91 \%\right)$

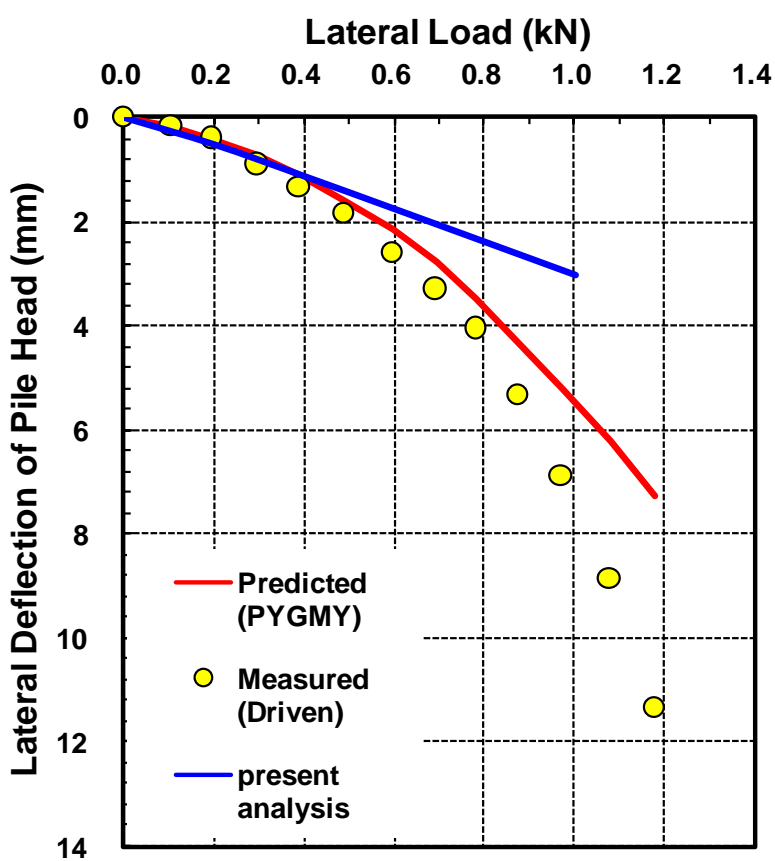

Medium dense sand $\left(D_{R}=59 \%\right)$

Loose sand $\left(D_{R}=38 \%\right)$
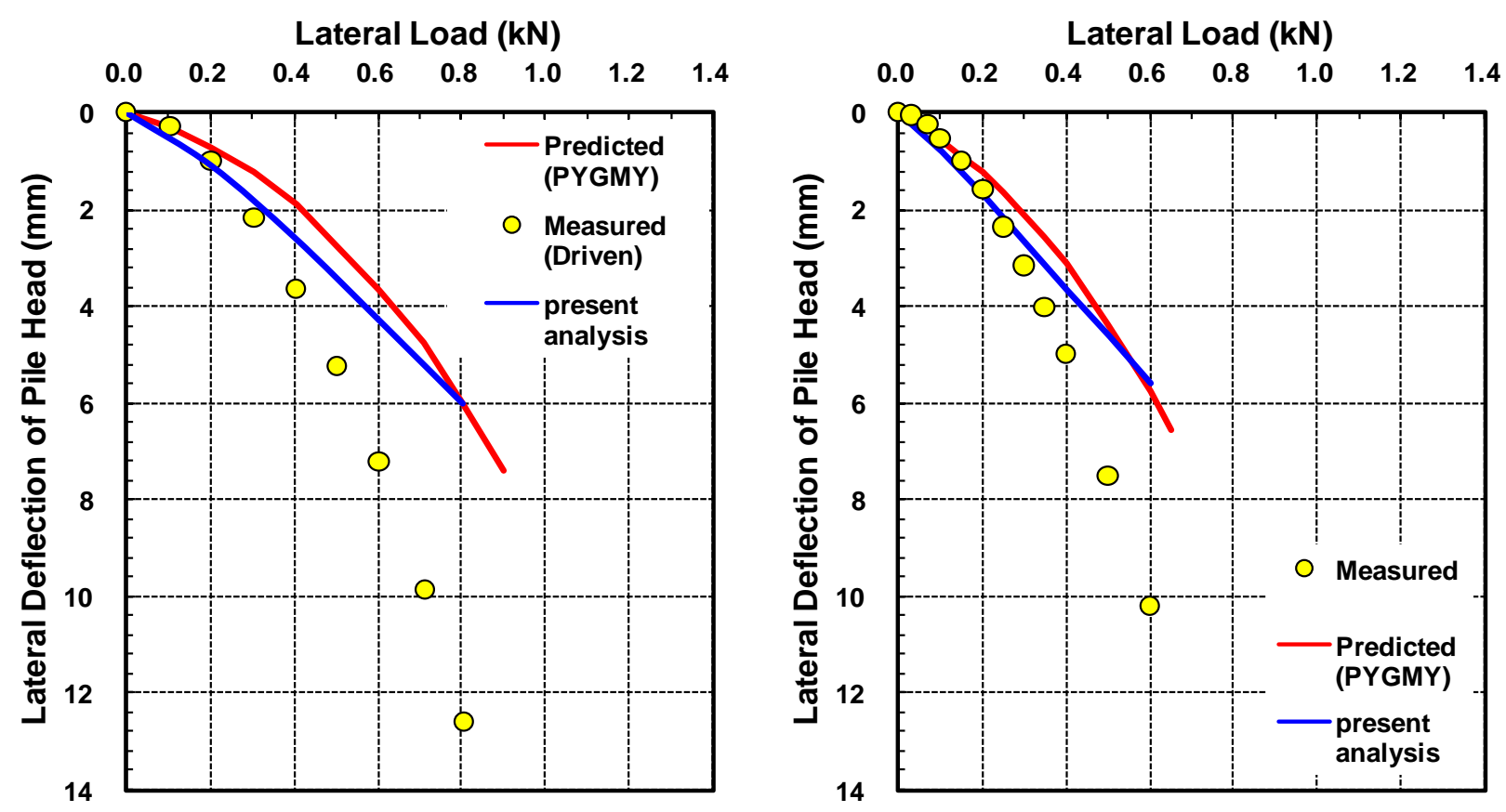


\section{Comparison of Result with Predictions}

- Comparison of predicted and measured bending moment profiles for model piles driven in dense sand
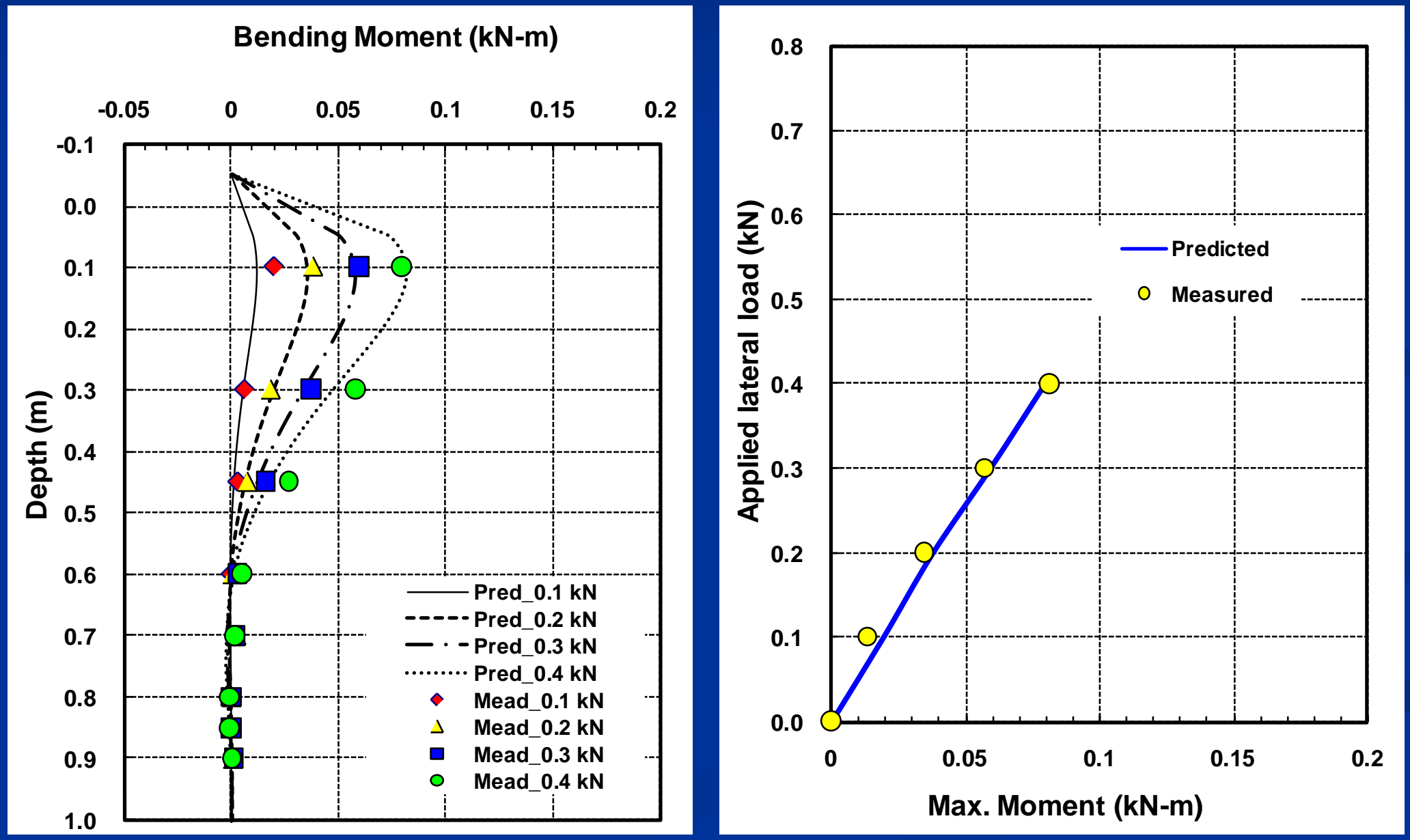


\section{Comparison of Results with PYGMY}

- Comparison of predicted and measured bending moment profiles for model piles driven in dense sand
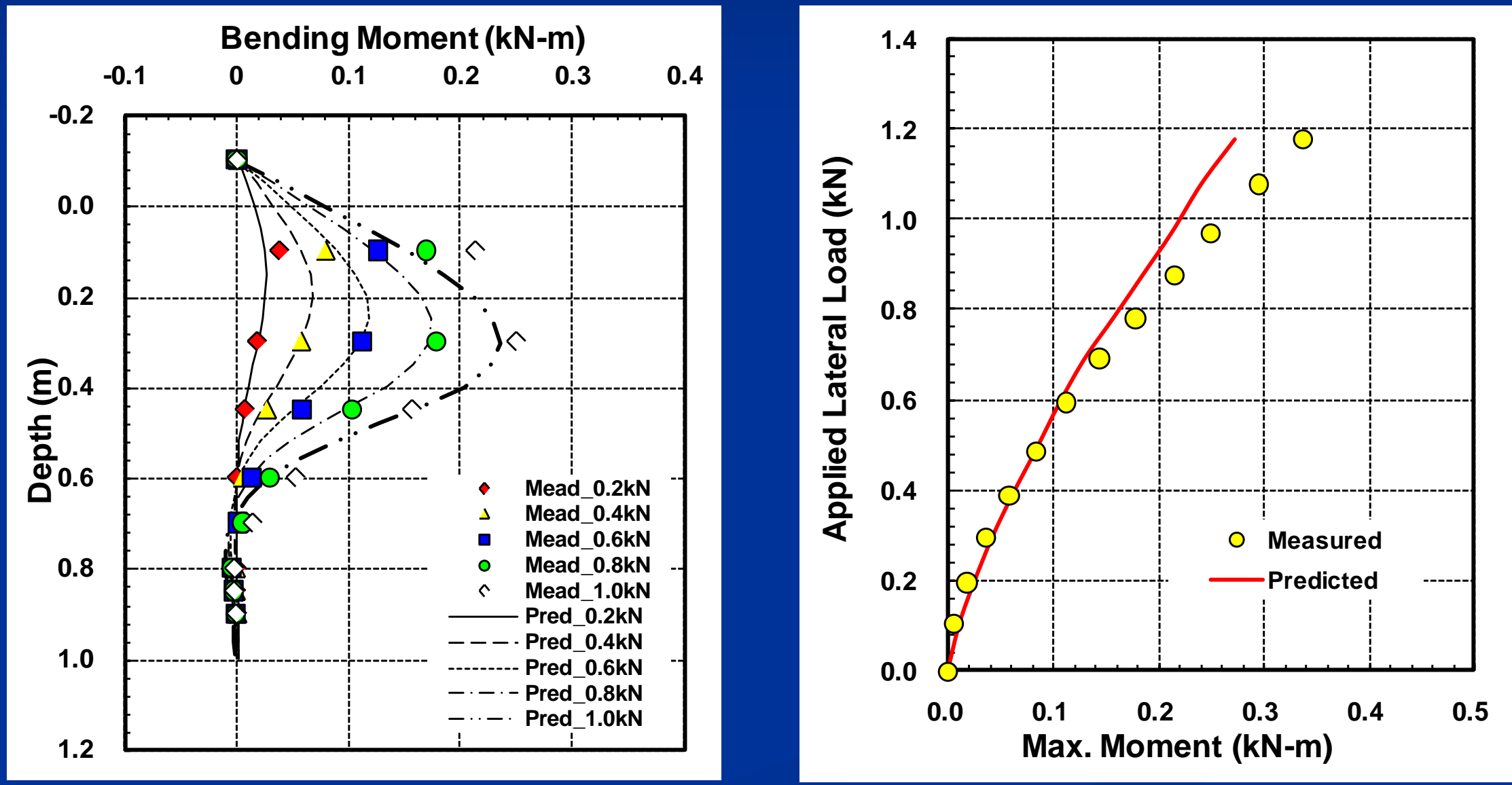


\section{Comparison of Results}

- Comparison of predictions with measurements from model piles driven and preinstalled in dense, medium dense

Dense sand $\left(D_{R}=91 \%\right)$

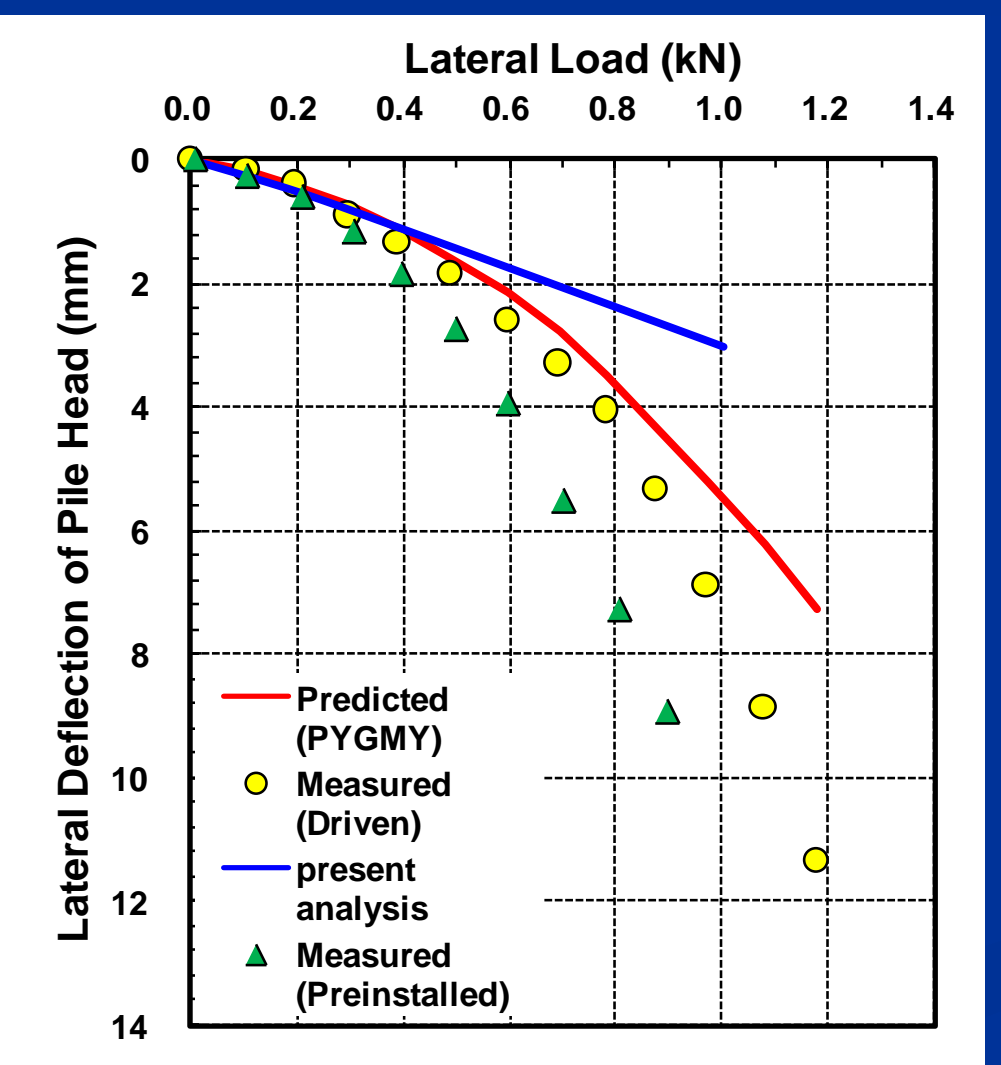

Medium dense sand $\left(D_{R}=59 \%\right)$

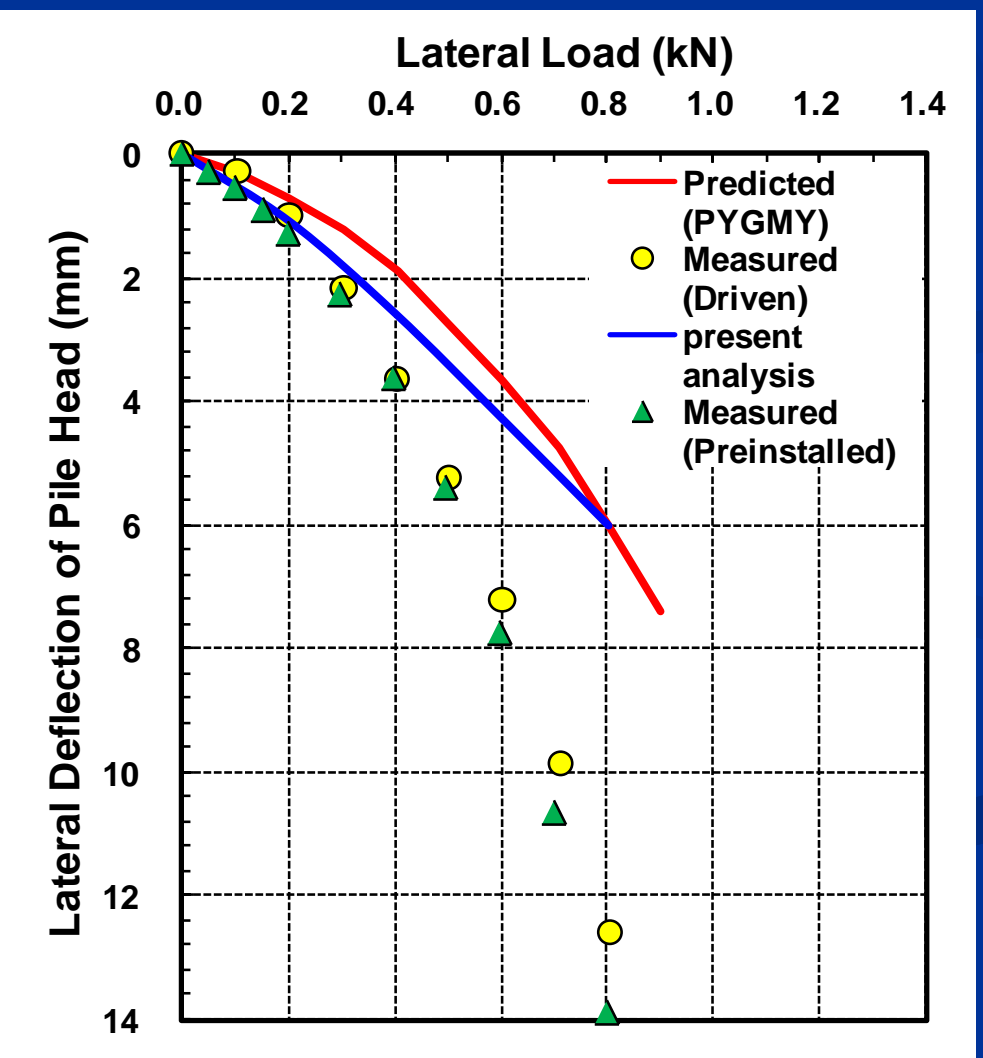




\section{Input parameters (present analysis)}

\begin{tabular}{|cc|}
\hline \multicolumn{2}{|c|}{ Input parameters for the model pile } \\
\hline Length (m) & 1.2 \\
\hline Diameter / width (m) & 0.03 \\
Pile modulus (GPa) & 210 \\
\hline \multicolumn{2}{|c|}{ Input parameters for the soil } \\
\hline Critical-state friction angle (deg.) & 32.8 \\
Max. void ratio ( $\left.\mathrm{e}_{\max }\right)$ & 0.78 \\
Min. void ratio ( $\left.\mathrm{e}_{\min }\right)$ & 0.47 \\
Coefficient of earth pressure & 0.4 \\
Model & $f-g$ model \\
\hline
\end{tabular}

-Suggested $f$ and $g$ (Lee and Salgado, 2000)

\begin{tabular}{ccc}
\hline $\mathbf{D}_{\mathbf{R}}(\mathbf{\%})$ & $\boldsymbol{f}$ & $\boldsymbol{g}$ \\
\hline 30 & 0.98 & 0.17 \\
50 & 0.97 & 0.2 \\
70 & 0.96 & 0.23 \\
90 & 0.95 & 0.26 \\
\hline
\end{tabular}




\section{Input parameters (PYGMY)}

\section{PYGMY input parameters for the model pile}

Pile property

Length (m)

Diameter / width (m)

Bending stiffness of pile, $\mathrm{E}_{\mathrm{p}} \mathrm{I}_{\mathrm{p}}\left(\mathrm{kN} \cdot \mathrm{m}^{2}\right)$

Plastic moment $(\mathrm{kN} \cdot \mathrm{m})$

No. of elements
Input value

1.2

0.03

3.862

0.5

30

\section{PYGMY input parameters for the soil}

\begin{tabular}{cccc} 
Soil property & Dense sand & $\begin{array}{c}\text { Medium dense } \\
\text { sand }\end{array}$ & Loose sand \\
Peak friction angle $(\mathrm{deg})$. & 44 & 39 & 36 \\
Initial stiffness gradient $(\mathrm{kPa} / \mathrm{m})$ & 82,000 & 34,000 & 12,000 \\
Effective unit weight $\left(\mathrm{kN} / \mathrm{m}^{3}\right)$ & 17.34 & 16.27 & 15.64 \\
No. of soil layers & 1 & 1 & 1 \\
\hline
\end{tabular}




\section{p-y curve (PYGMY)}

- Sand: API criterion (1993)

$$
p=A \cdot p_{u} \cdot \tanh \left(\frac{k \cdot x \cdot y}{A \cdot p_{u} \cdot B}\right)
$$

$p$ : lateral pressure

$A$ : factor to account for static or cyclic loading

$$
\begin{aligned}
& A=0.9 \text { where equilibrium has been reached under cyclic loading } \\
& A=(0.3-0.8 x / B) \geq 0.9 \text { for static loading }
\end{aligned}
$$

$p_{u}=$ fultimate bearing pressure at the current depth, $x$

$k=$ gradient of initial modulus of subgrade reaction with depth $(\mathrm{kPa} / \mathrm{m})$

$y=$ lateral displacement

$x=$ depth below surface

$B=$ pile diameter 


\section{p-y curves (PYGMY)}

- predicted p-y curves (dense sand)

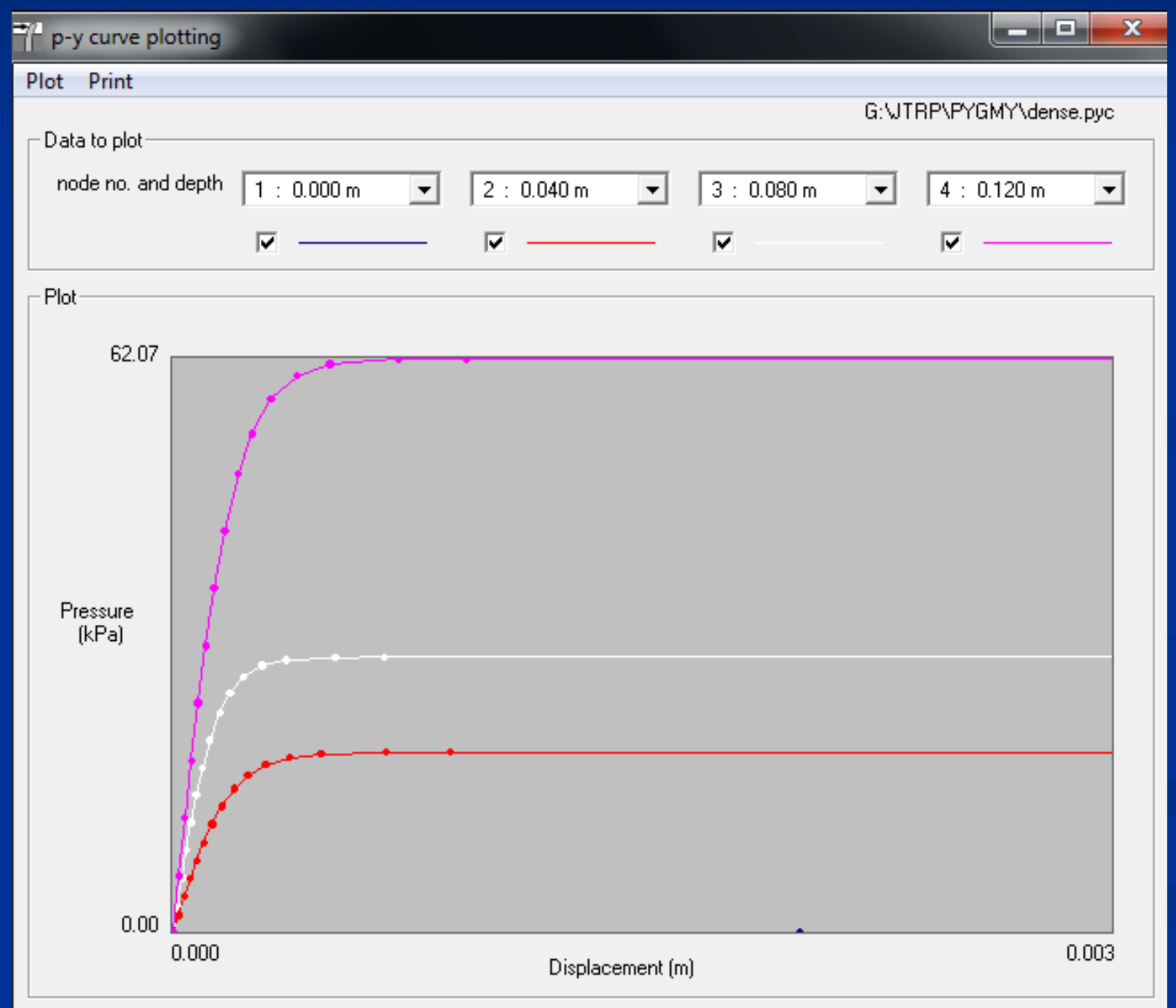




\section{Contents}

- Model pile testing plan

- Model pile testing

- Sample preparation

- Soil tank

- Sand pluviator

- Instrumented model pile

- Driving system

- Preinstallation method

- Jacking system

- Installation of piles for pile group testing

- Lateral loading system

- Comparison of test and analysis results

- Single piles

- Pile groups

- Summary and Conclusions 


\section{Group piles: Dense sand $\left(D_{R}=90 \%\right)$}

- Lateral deflection of pile head (4 piles, 3B spacing)

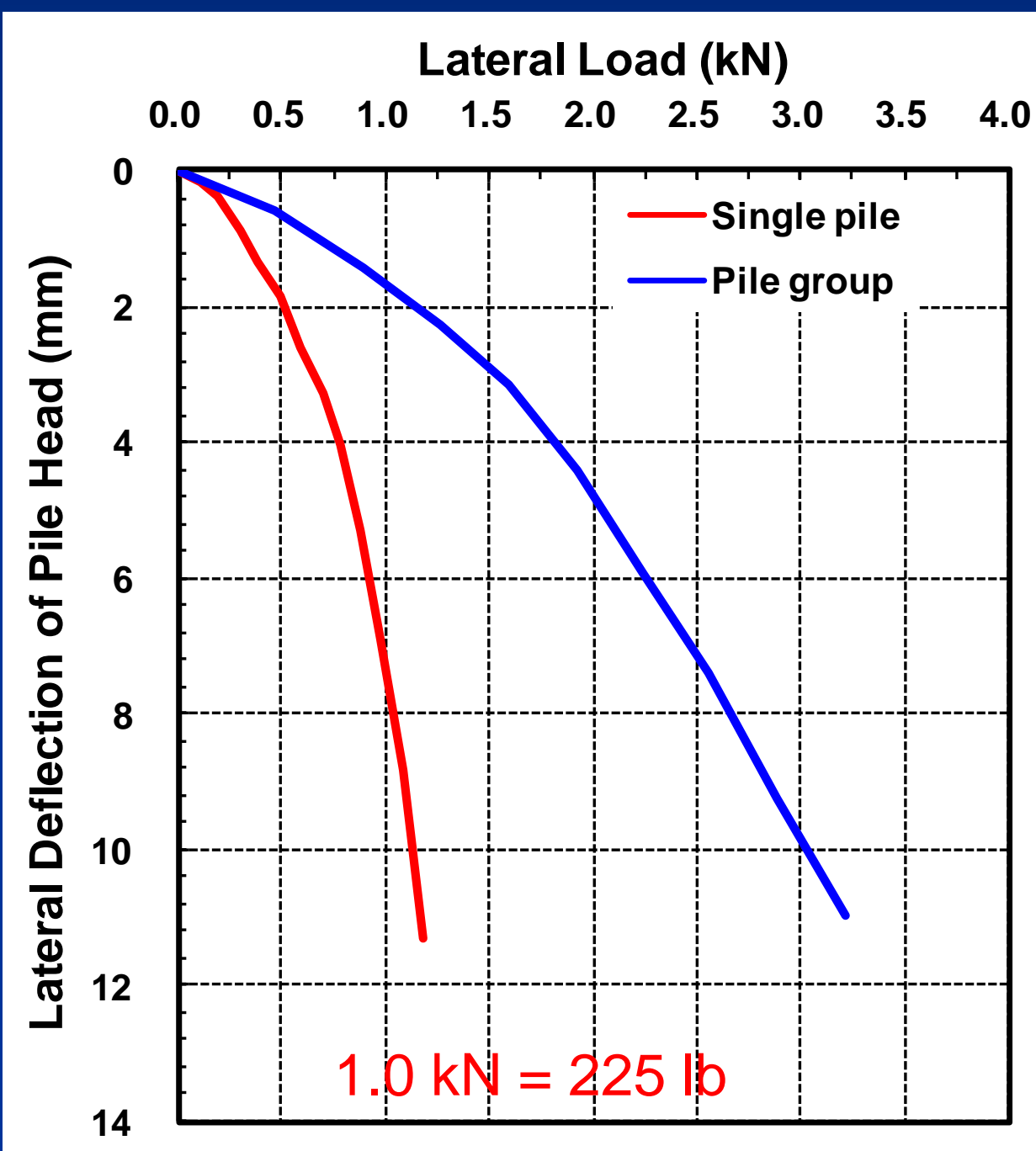

Lateral deflection

$=5 \%, 10 \%, 20 \%$ of the pile diameter

$$
\begin{aligned}
& \mathrm{Q}_{\text {lat, } 5 \%}=0.91 \mathrm{kN} \\
& \mathrm{Q}_{\text {lat, } 10 \%}=1.59 \mathrm{kN} \\
& \mathrm{Q}_{\text {lat, } 20 \%}=2.25 \mathrm{kN}
\end{aligned}
$$

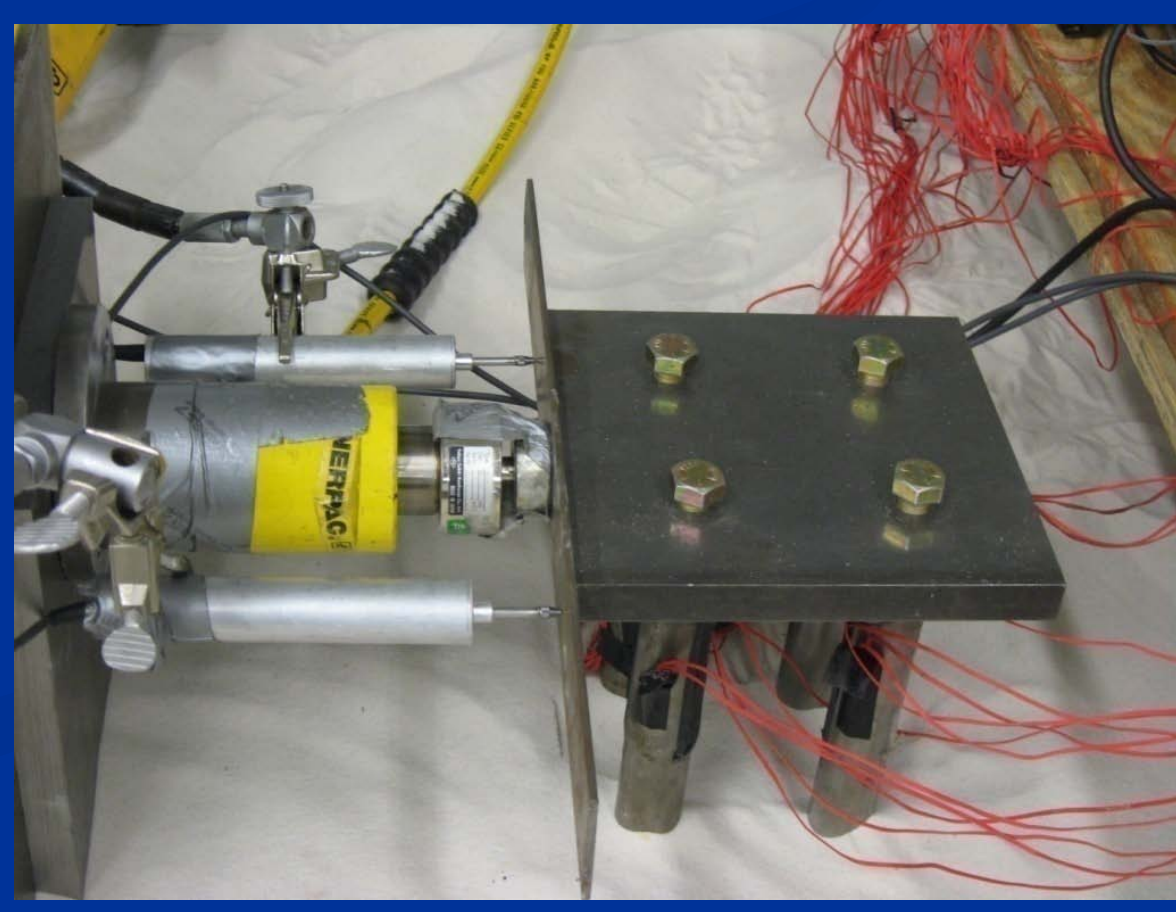




\section{Group piles: Dense sand $\left(D_{R}=90 \%\right)$}

- Distribution of lateral load
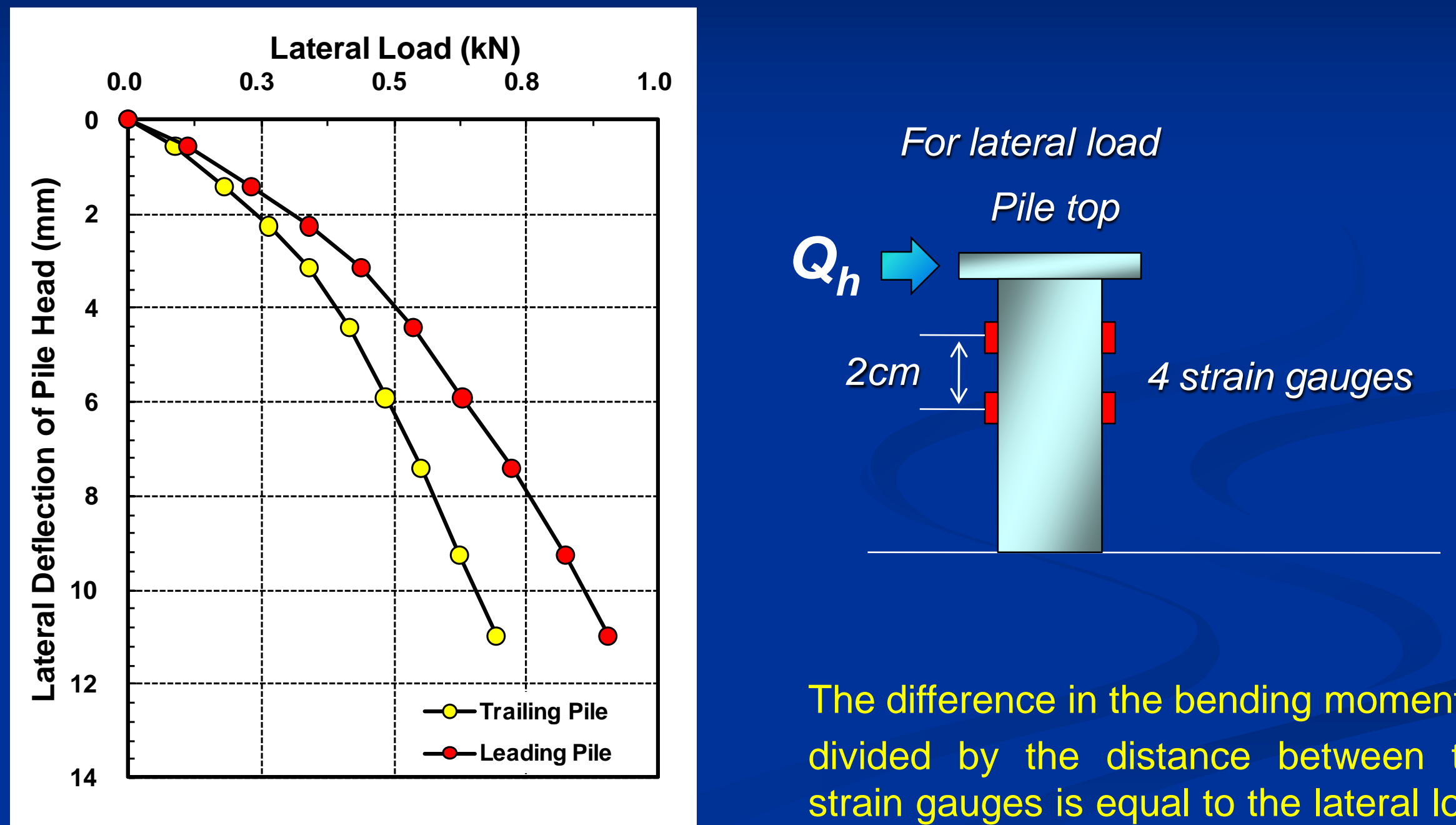

The difference in the bending moments divided by the distance between the strain gauges is equal to the lateral load in the pile 


\section{Group piles: Dense sand $\left(D_{R}=90 \%\right)$}

- Bending moment of leading and trailing piles

Distribution of bending moments of trailing piles

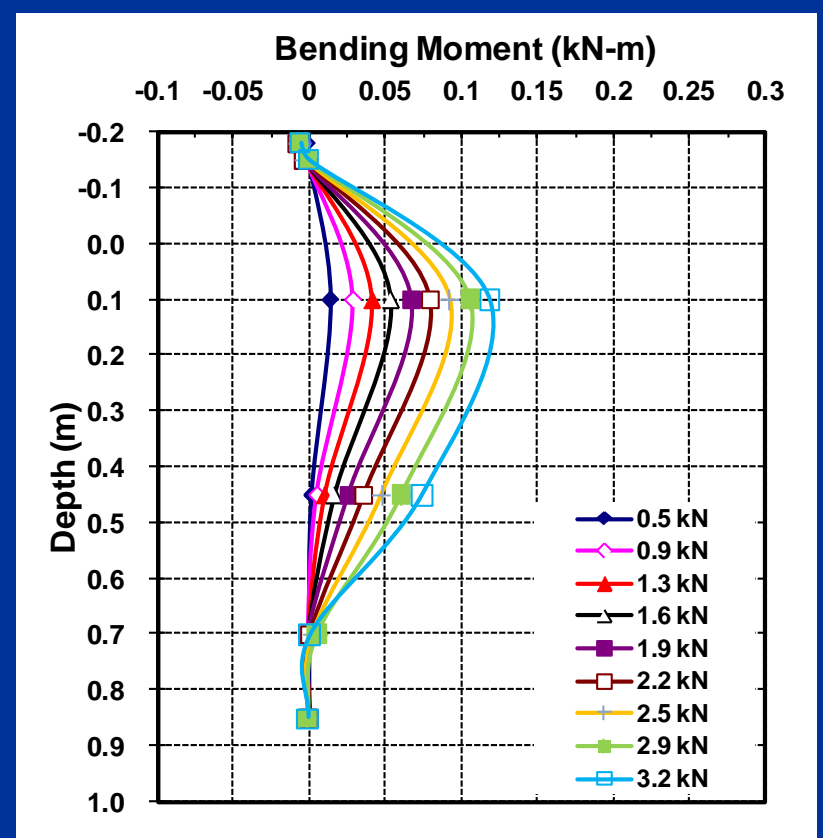

Distribution of bending moments of leading piles

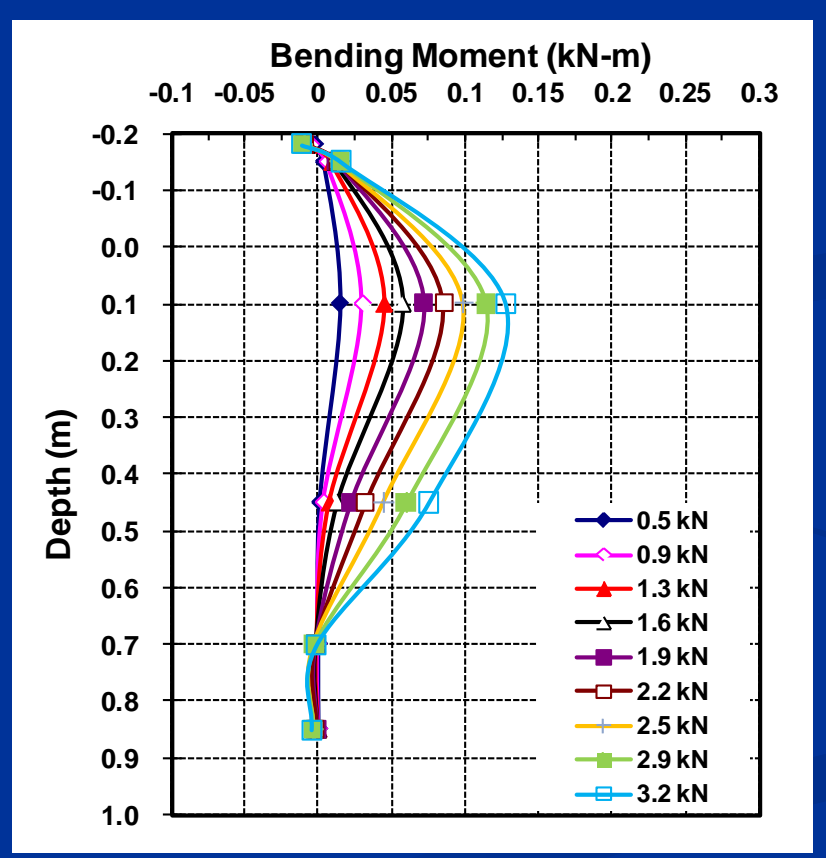

\section{Group piles}

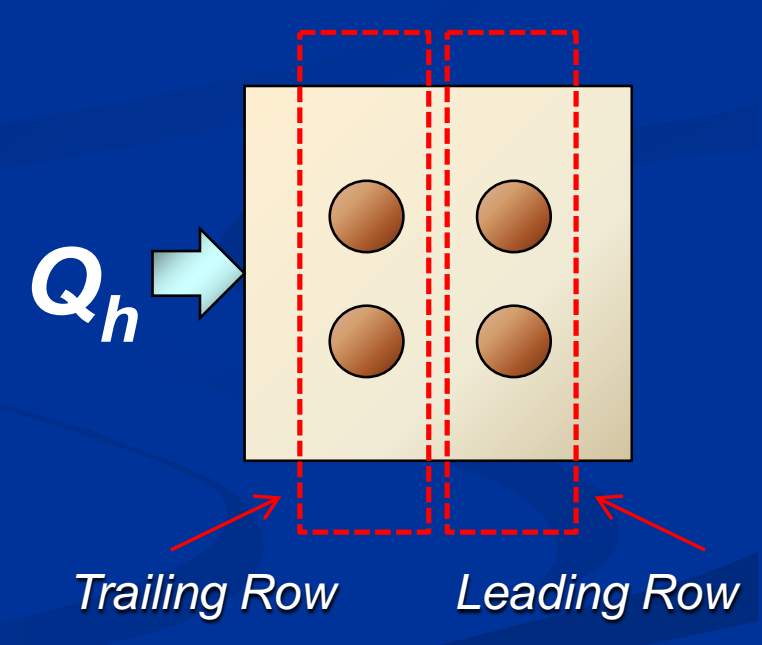




\section{Group piles: Dense sand $\left(D_{R}=90 \%\right)$}

- Measured p-multipliers and distribution of lateral load

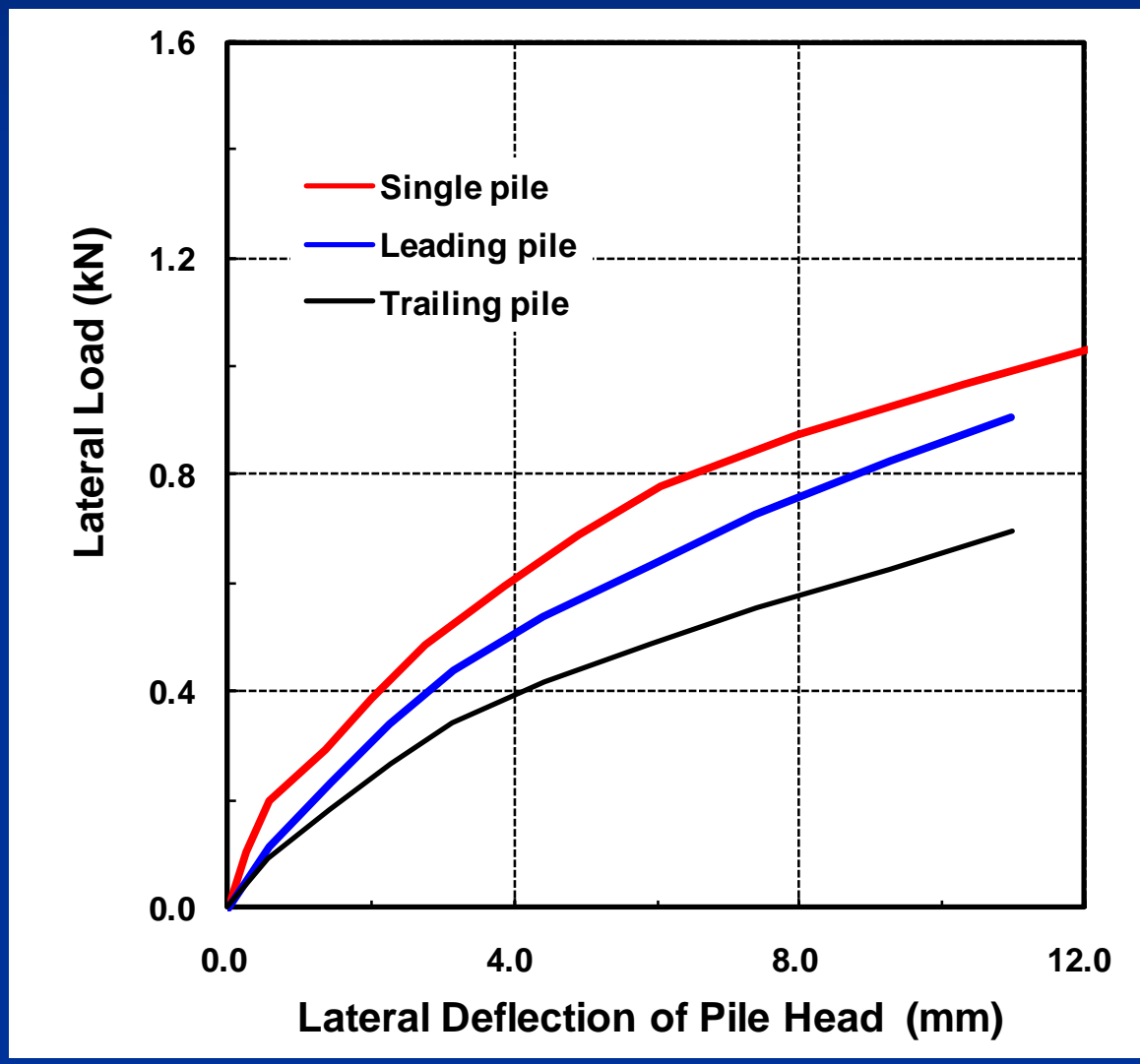

Measured $p$-multipliers

\begin{tabular}{ccc} 
Lateral deflection & $\begin{array}{c}\text { Leading } \\
\text { Pile }\end{array}$ & $\begin{array}{c}\text { Trailing } \\
\text { Pile }\end{array}$ \\
\hline \hline $5 \%$ of B & 0.76 & 0.59 \\
$10 \%$ of B & 0.74 & 0.58 \\
$20 \%$ of B & 0.82 & 0.63 \\
\hline
\end{tabular}




\section{Group piles: Medium dense sand $\left(D_{R}=60 \%\right)$}

- Lateral deflection of pile head (4 piles, 3B spacing)

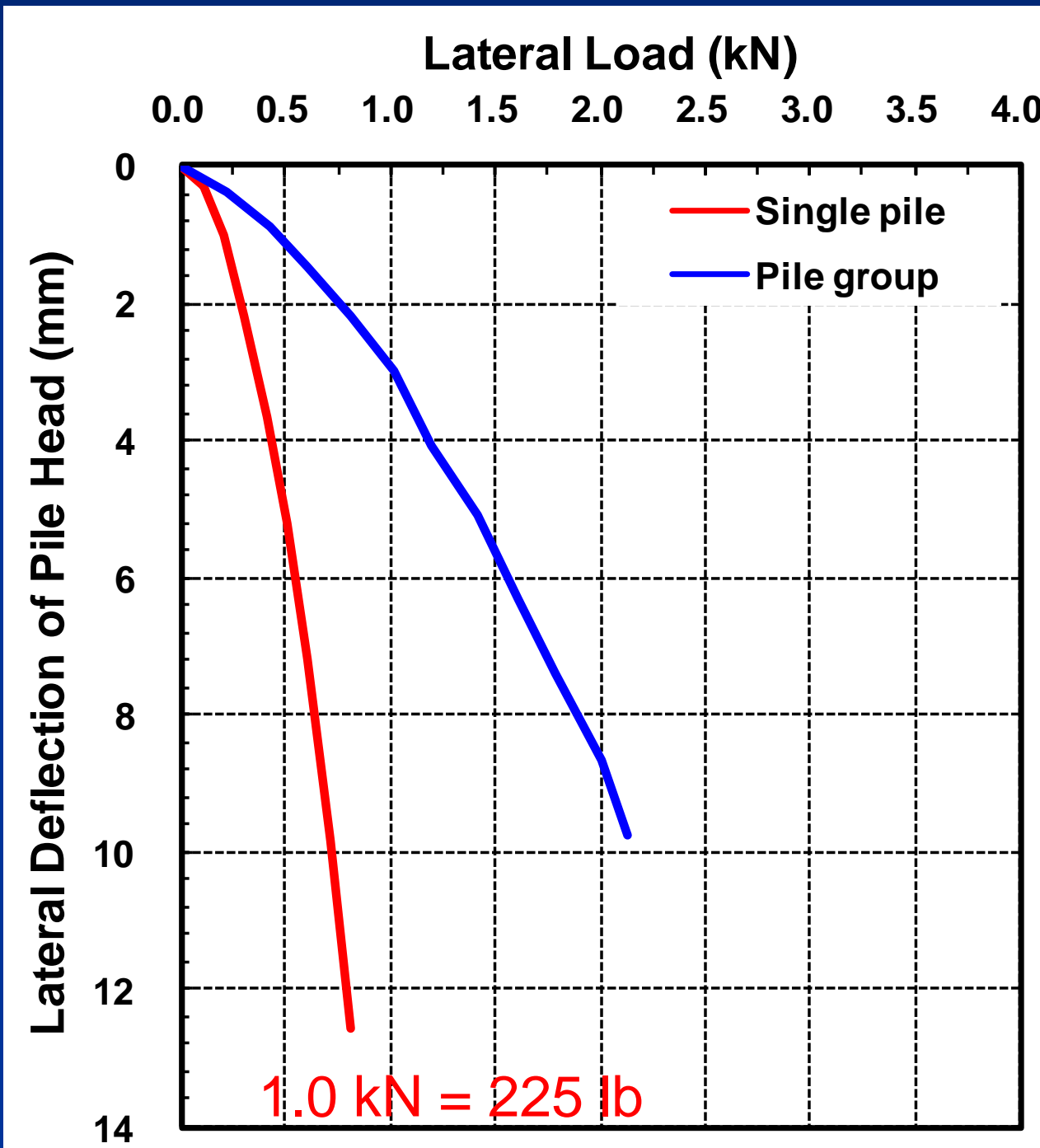

Lateral deflection

$=5 \%, 10 \%, 20 \%$ of the pile diameter

$\mathrm{Q}_{\text {lat, 5\% }}=0.60 \mathrm{kN}$

$\mathrm{Q}_{\text {lat, } 10 \%}=1.20 \mathrm{kN}$

$\mathrm{Q}_{\text {lat, } 20 \%}=1.56 \mathrm{kN}$ 


\section{Group piles: Medium dense sand $\left(D_{R}=60 \%\right)$}

- Bending moment of leading and trailing piles

Distribution of bending moments of trailing piles

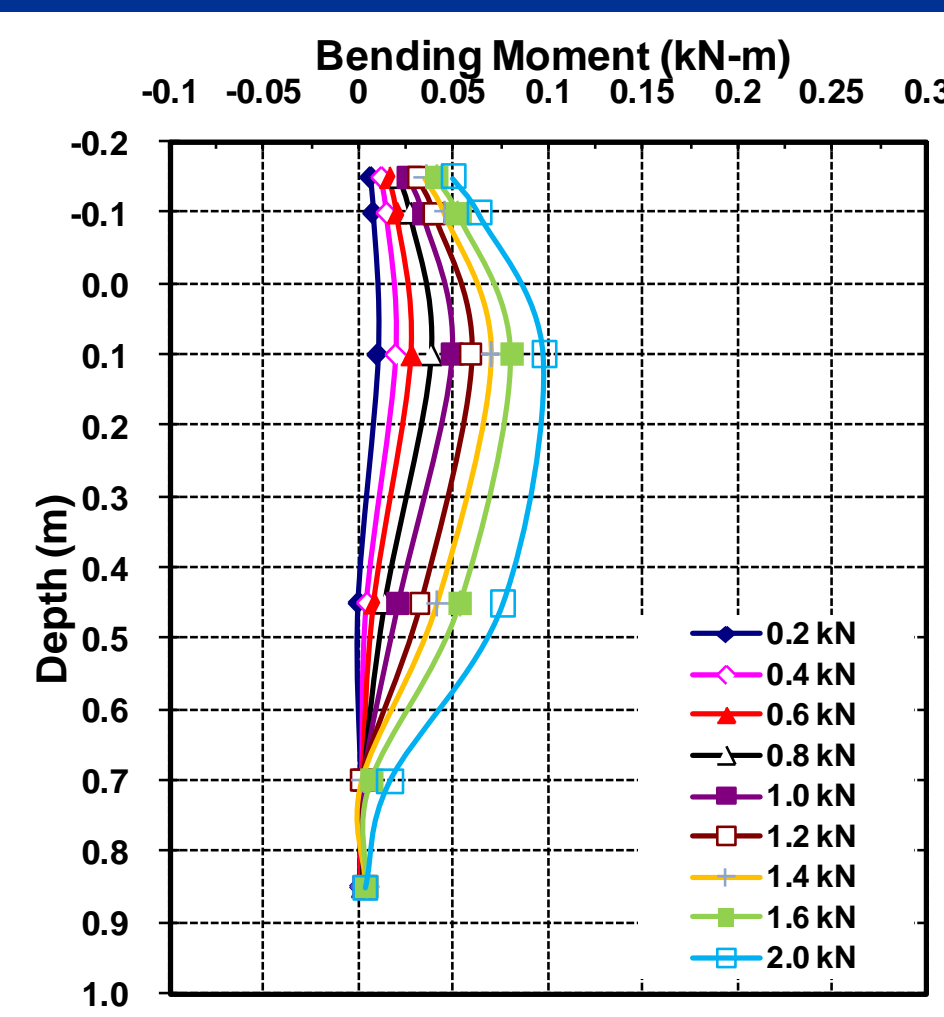

Distribution of bending moments of leading piles

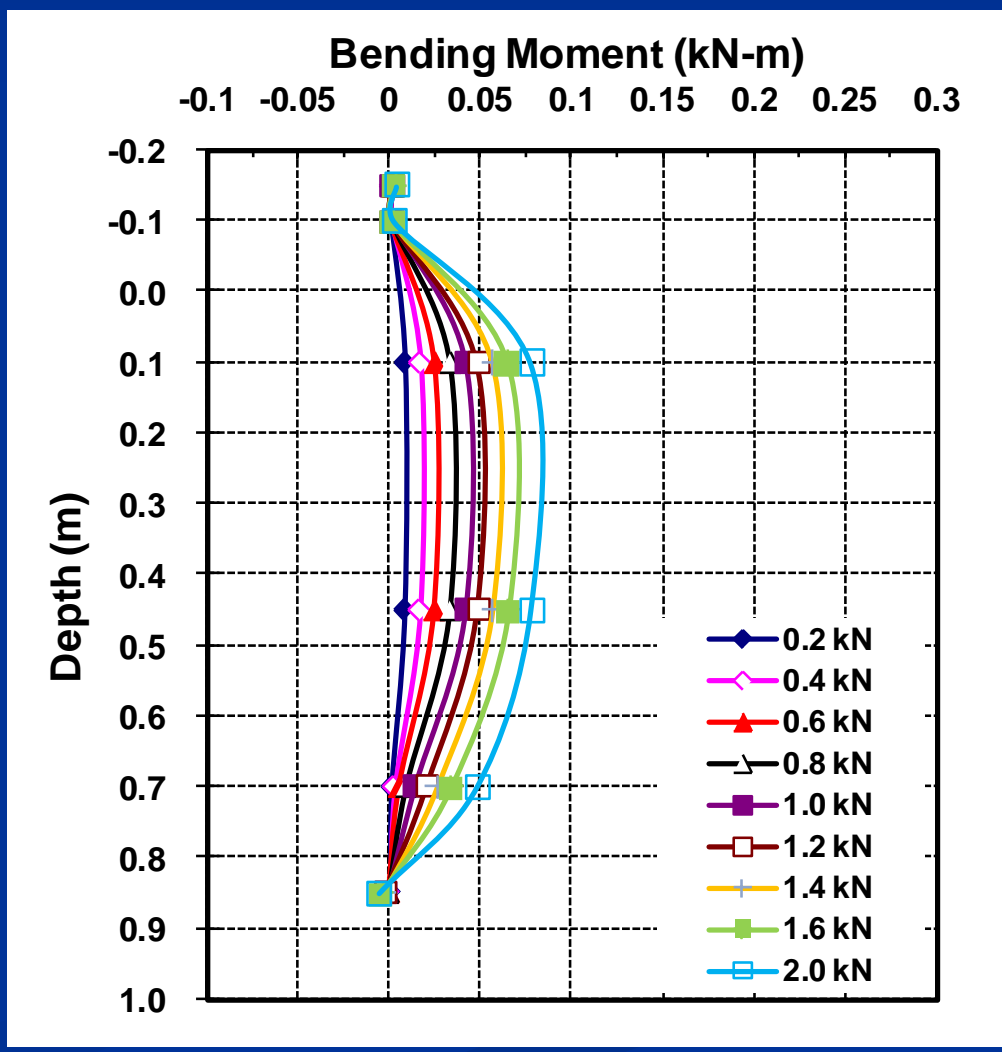




\section{Group piles: Medium dense sand $\left(D_{R}=60 \%\right)$}

- Measured p-multipliers and distribution of lateral load

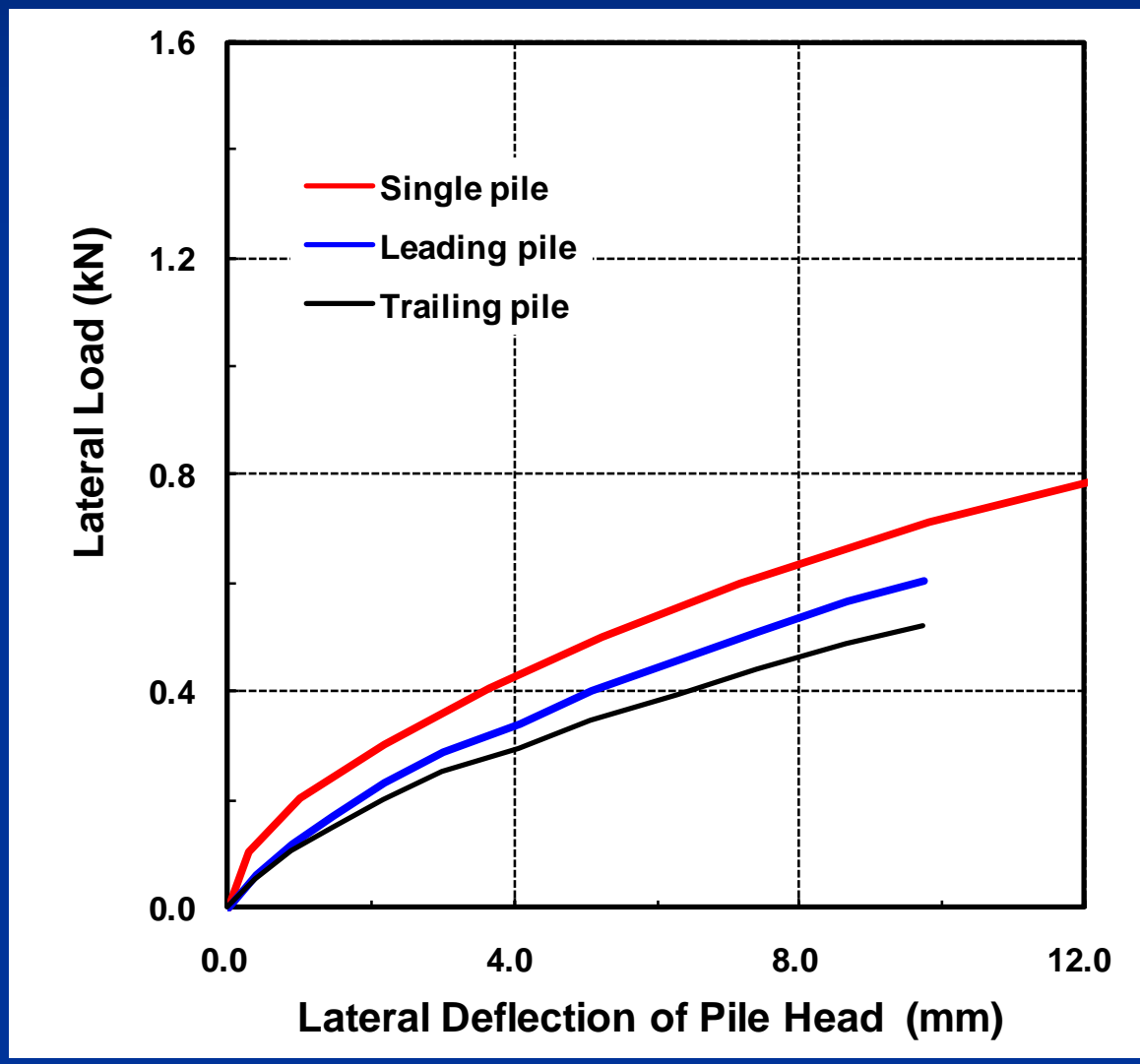

Measured $p$-multipliers

\begin{tabular}{ccc} 
Lateral deflection & $\begin{array}{c}\text { Leading } \\
\text { Pile }\end{array}$ & $\begin{array}{c}\text { Trailing } \\
\text { Pile }\end{array}$ \\
\hline \hline $5 \%$ of B & 0.70 & 0.60 \\
$10 \%$ of B & 0.68 & 0.59 \\
$20 \%$ of B & 0.82 & 0.71 \\
\hline
\end{tabular}




\section{Group piles: Loose sand $\left(D_{R}=40 \%\right)$}

- Lateral deflection of pile head (4 piles, 3B spacing)

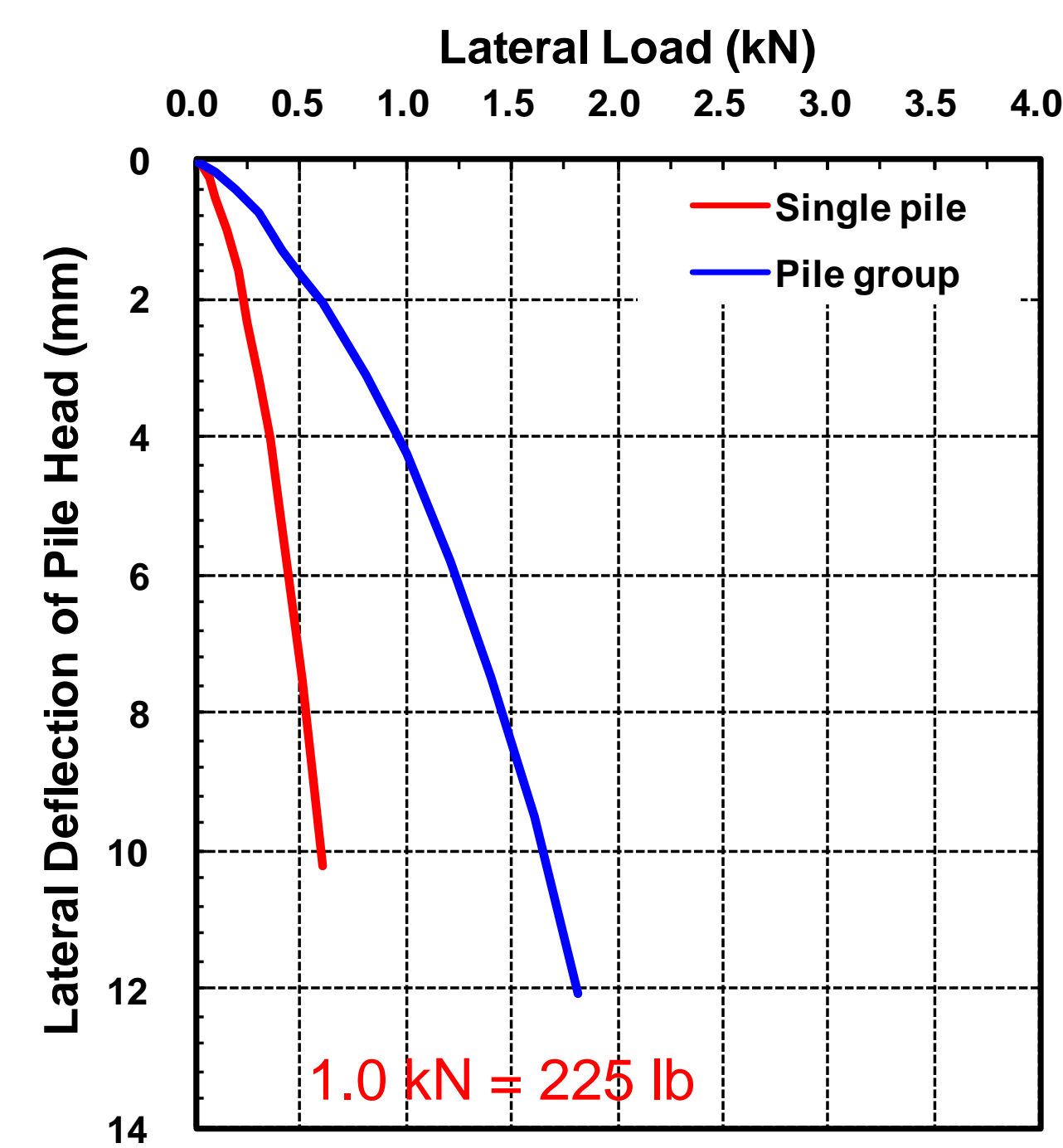

Lateral deflection

$=5 \%, 10 \%, 20 \%$ of the pile diameter

$$
\begin{aligned}
& \mathrm{Q}_{\text {lat, 5\% }}=0.47 \mathrm{kN} \\
& \mathrm{Q}_{\text {lat, } 10 \%}=0.80 \mathrm{kN} \\
& \mathrm{Q}_{\text {lat, 20\% }}=1.23 \mathrm{kN}
\end{aligned}
$$




\section{Group piles: Loose sand $\left(D_{R}=40 \%\right)$}

- Bending Moment of leading and trailing piles

Distribution of bending moments of trailing piles

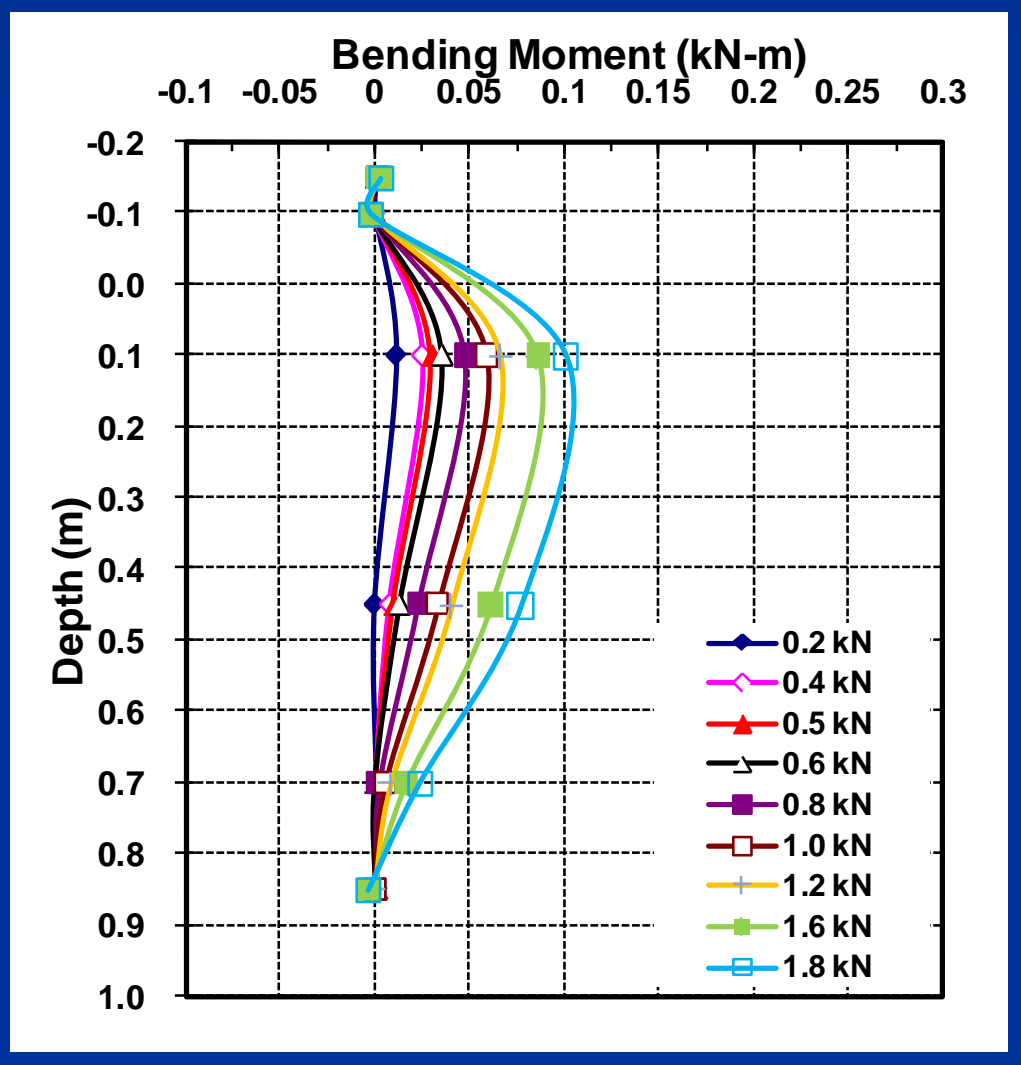

Distribution of bending moments of leading piles

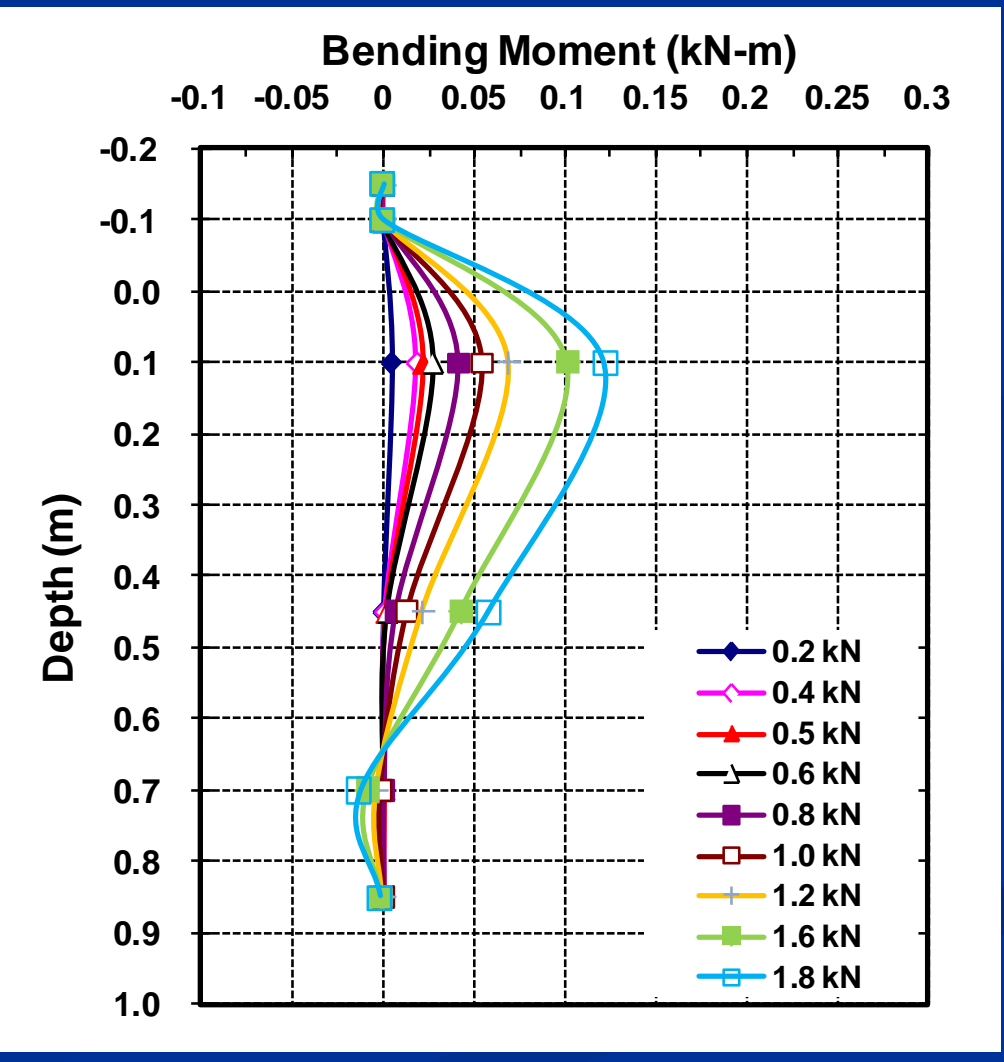




\section{Group piles: Loose sand $\left(D_{R}=40 \%\right)$}

- Measured p-multipliers and distribution of lateral load

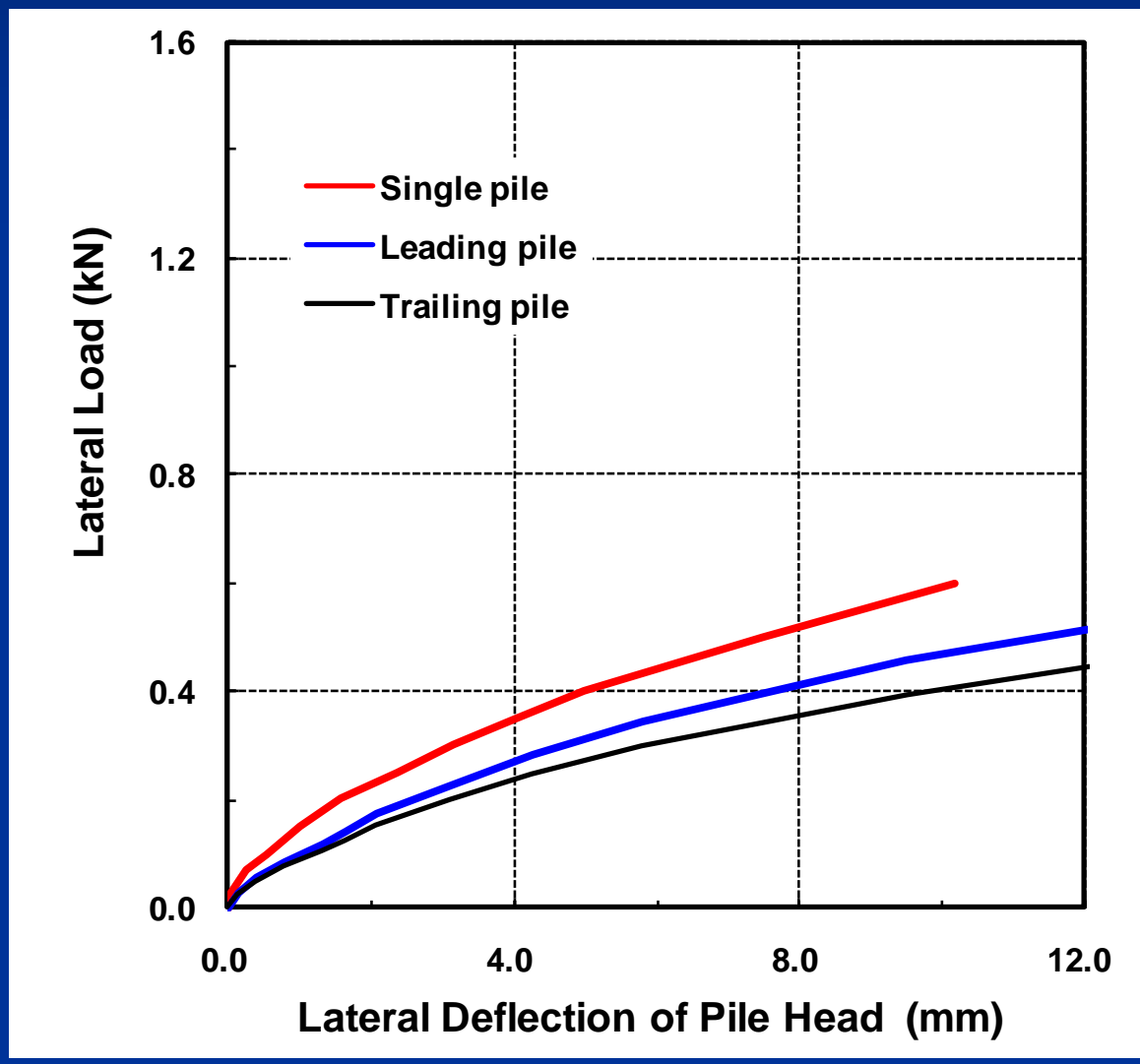

Measured $p$-multipliers

\begin{tabular}{ccc|} 
Lateral deflection & $\begin{array}{c}\text { Leading } \\
\text { Pile }\end{array}$ & $\begin{array}{c}\text { Trailing } \\
\text { Pile }\end{array}$ \\
\hline \hline $5 \%$ of B & 0.68 & 0.59 \\
$10 \%$ of B & 0.76 & 0.65 \\
$20 \%$ of B & 0.80 & 0.69 \\
\hline
\end{tabular}




\section{Group piles: two-layer sand sample}

- Lateral deflection of pile head (4 piles, 3B spacing)
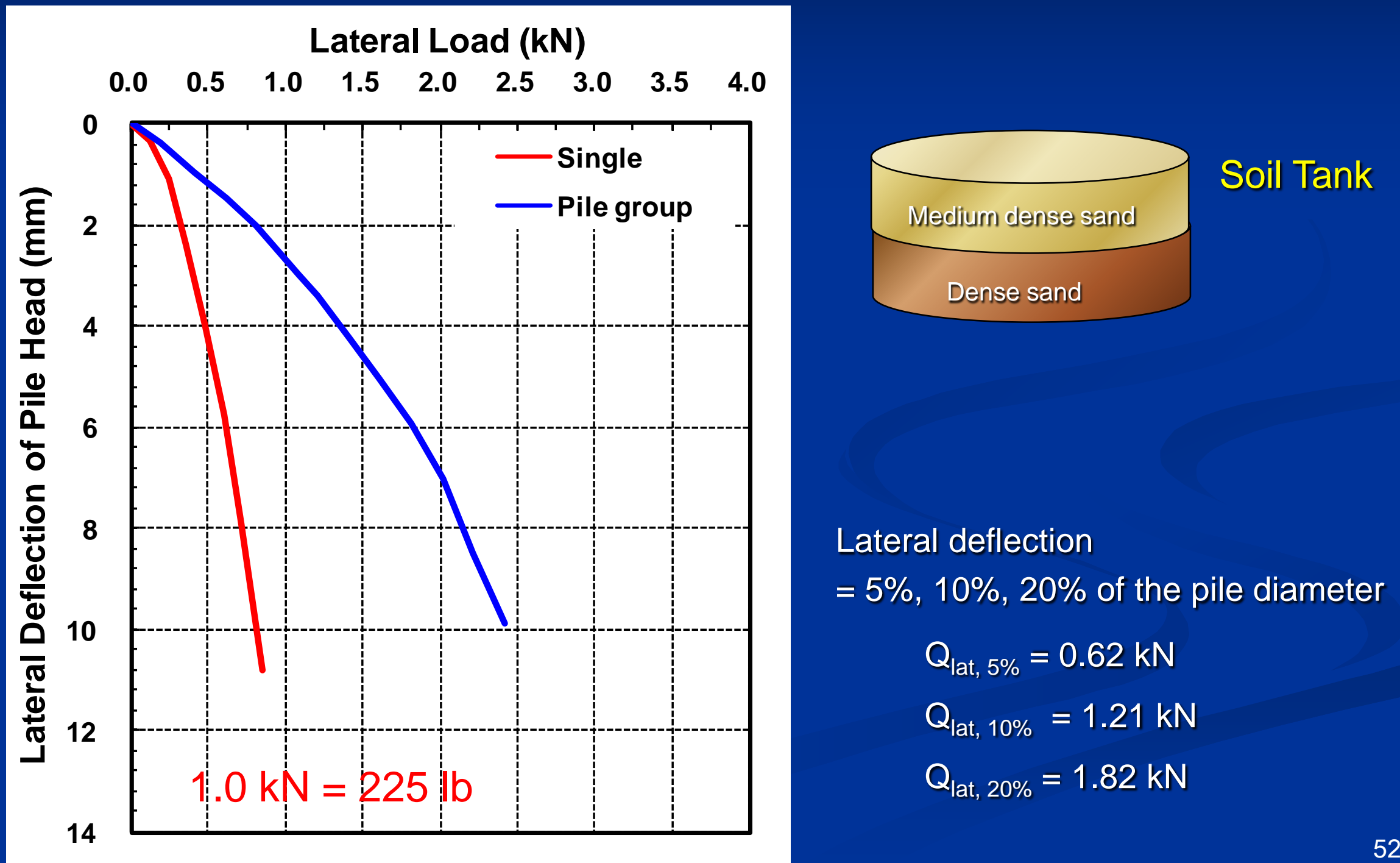

Lateral deflection

$=5 \%, 10 \%, 20 \%$ of the pile diameter

$$
\begin{aligned}
& \mathrm{Q}_{\text {lat, 5\% }}=0.62 \mathrm{kN} \\
& \mathrm{Q}_{\text {lat, } 10 \%}=1.21 \mathrm{kN} \\
& \mathrm{Q}_{\text {lat, 20\% }}=1.82 \mathrm{kN}
\end{aligned}
$$




\section{Group piles: two-layer sand sample}

- Bending Moment of leading and trailing piles

Distribution of bending moments of trailing piles

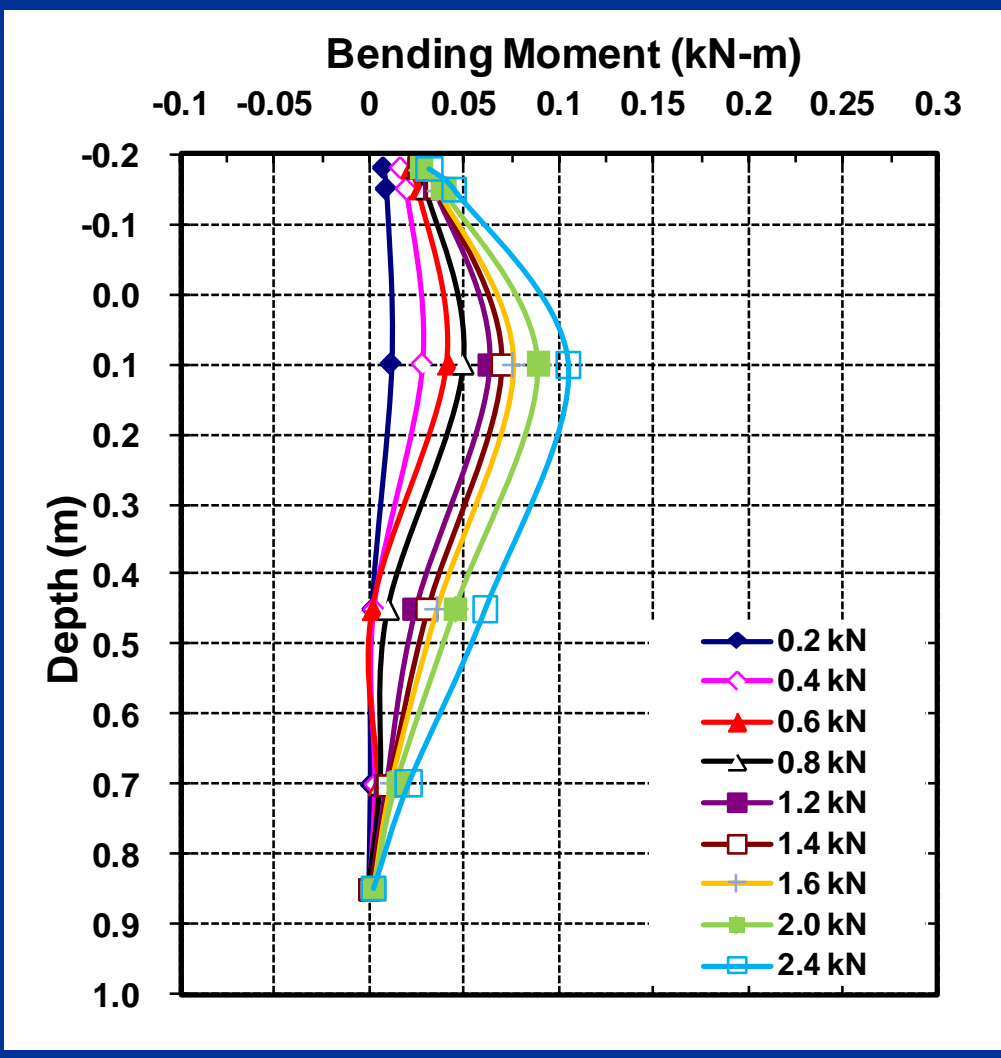

Distribution of bending moments of leading piles

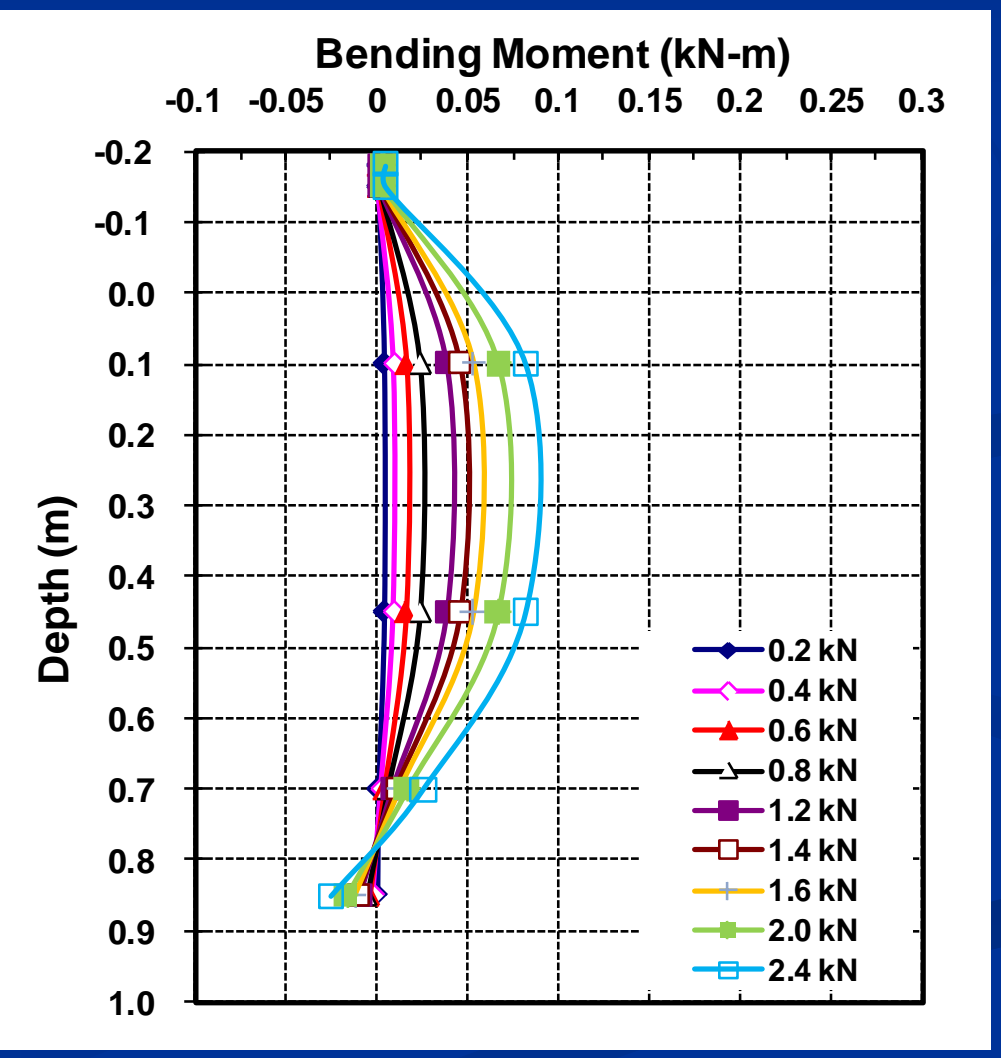




\section{Group piles: two-layer sand sample}

- Measured p-multipliers and distribution of lateral load

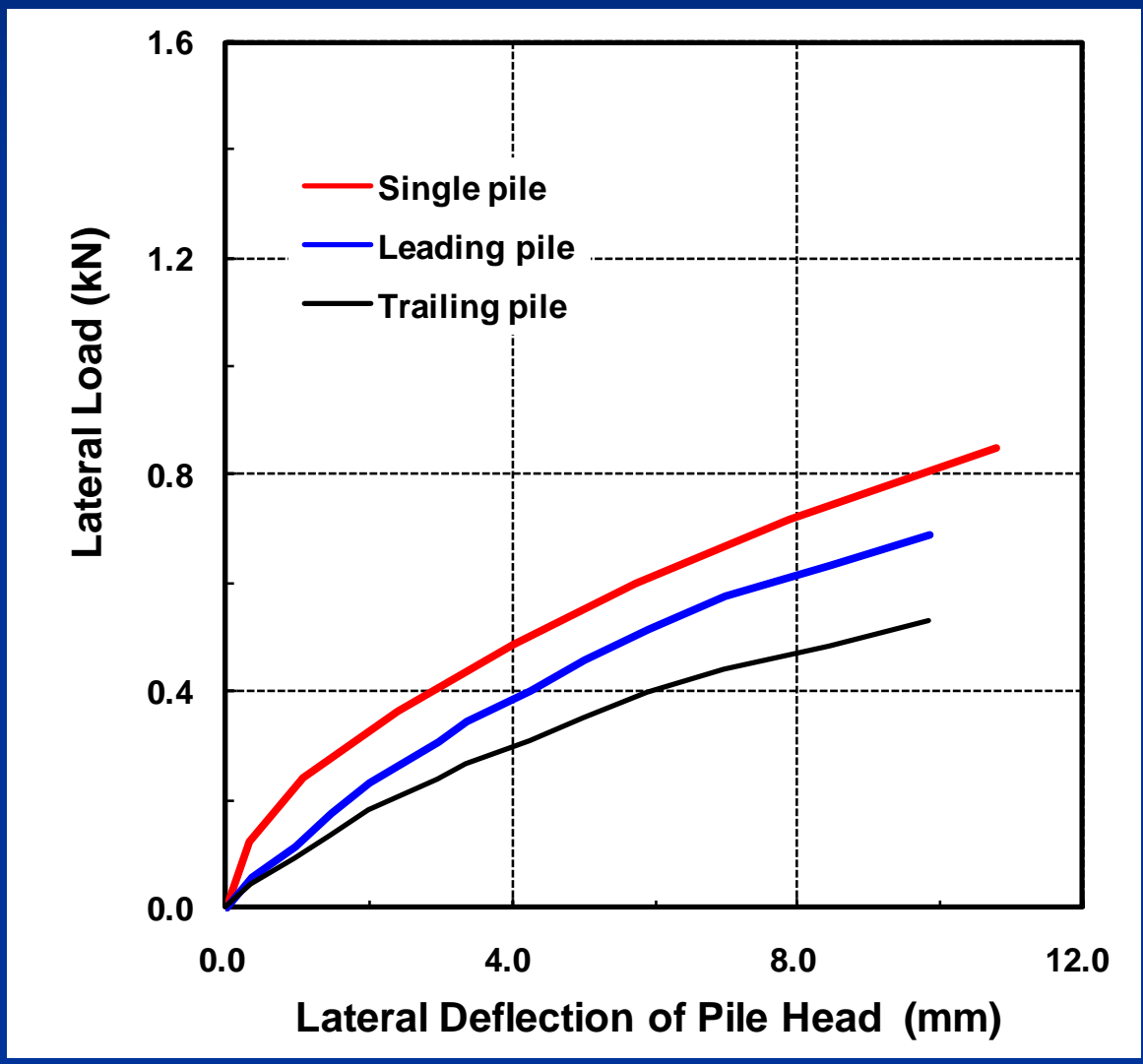

Measured $p$-multipliers

\begin{tabular}{ccc} 
Lateral deflection & $\begin{array}{c}\text { Leading } \\
\text { Pile }\end{array}$ & $\begin{array}{c}\text { Trailing } \\
\text { Pile }\end{array}$ \\
\hline \hline $5 \%$ of B & 0.63 & 0.49 \\
$10 \%$ of B & 0.71 & 0.55 \\
$20 \%$ of B & 0.84 & 0.65
\end{tabular}




\section{Group piles: $\boldsymbol{p}$-multipliers}

- p-multipliers $f$

- $p-y$ relationship for single pile

- Reduction in $p$ values for each pile in the group by using $f$

Reese et al (2006)

$$
\begin{aligned}
& f_{i}=\prod_{j=1}^{n_{p}} \beta_{i j} \\
& \beta_{i j}: \text { interaction coefficients (pile } i \text { and pile } j \text { ) } \\
& \beta_{i j}=1 \text { if } i=j
\end{aligned}
$$




\section{Group piles: $\boldsymbol{p}$-multipliers}

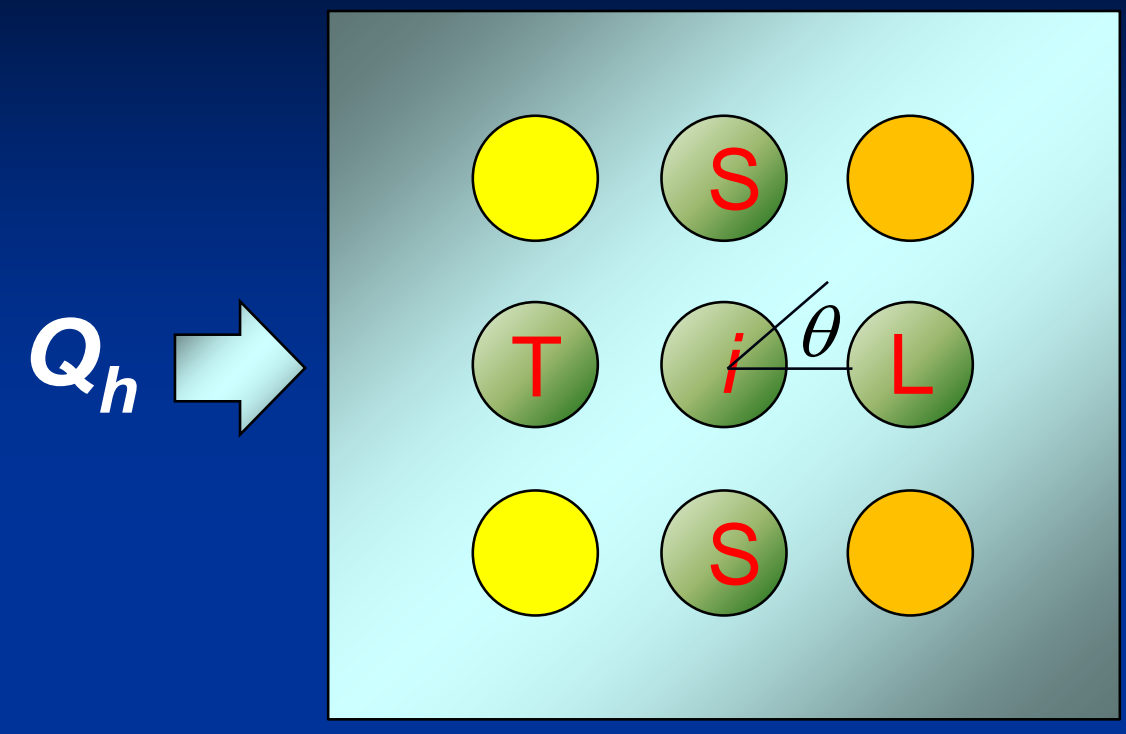

leading offset pile

trailing offset pile

$S_{p i L}, S_{p i T}, S_{p i S}:$

center-to-center spacing

$B$ : pile diameter

$\beta_{i L}=0.48\left(\frac{s_{p i L}}{B}\right)^{0.38} \leq 1$ for a leading pile

$\beta_{i \theta}=\sqrt{\beta_{i L}^{2} \cos ^{2} \theta+\beta_{i S}^{2} \sin ^{2} \theta}$

$\beta_{i T}=0.7\left(\frac{s_{p i T}}{B}\right)^{0.26} \leq 1 \quad$ for a trailing pile

for leading offset pile

$\beta_{i T}=0.64\left(\frac{s_{p i s}}{B}\right)^{0.34} \leq 1$ for a side-by-side pile

$\beta_{i \theta}=\sqrt{\beta_{i T}^{2} \cos ^{2} \theta+\beta_{i S}^{2} \sin ^{2} \theta}$ for trailing offset pile 


\section{Group piles: $\boldsymbol{p}$-multipliers}

for a leading pile

$\beta_{12}=0.48\left(\frac{s_{p i L}}{B}\right)^{0.38}=0.48\left(\frac{0.09}{0.03}\right)^{0.38}=0.72$

for a sicle-by-side pile

$\beta_{13}=0.64\left(\frac{s_{p i T}}{B}\right)^{0.34}=0.64\left(\frac{0.09}{0.03}\right)^{0.34}=0.92$

for leadjing offiset pile

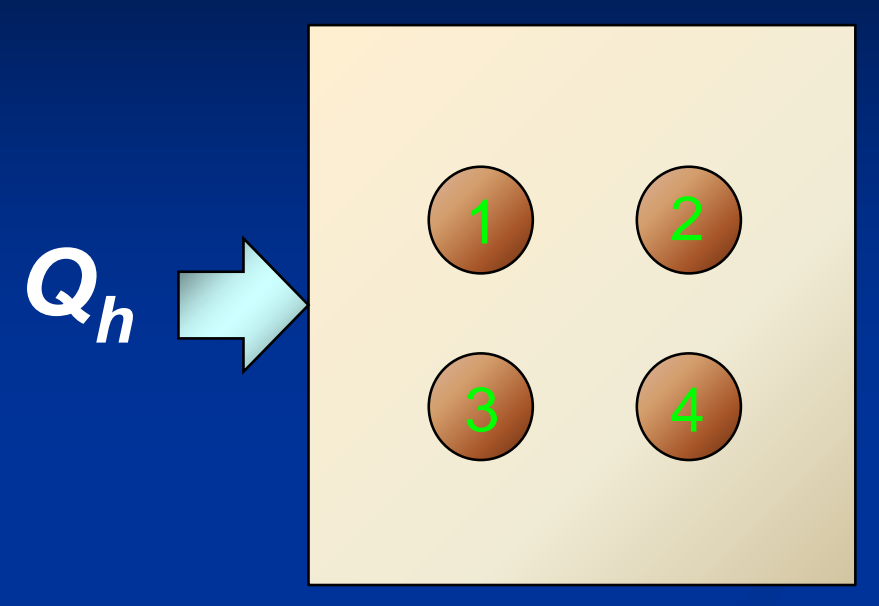

4 piles, 3B spacing $(=0.09 \mathrm{~m})$

$\beta_{14}=\sqrt{\beta_{1 L}^{2} \cos ^{2} \theta+\beta_{1 S}^{2} \sin ^{2} \theta}$

$\beta_{1 L}=0.48\left(\frac{s_{p i L}}{B}\right)^{0.38}=0.48\left(\frac{0.09 \cdot \sqrt{2}}{0.03}\right)^{0.38}=0.83$

$\beta_{1 S}=0.64\left(\frac{s_{p i L}}{B}\right)^{0.34}=0.64\left(\frac{0.09 \cdot \sqrt{2}}{0.03}\right)^{0.34}=1.04 \rightarrow 1$

$\beta_{14}=\sqrt{\beta_{1 L}^{2} \cos ^{2} \theta+\beta_{1 S}^{2} \sin ^{2} \theta}=0.919$

$$
f_{1}=\prod_{j=1}^{n_{p}} \beta_{i j}=\beta_{12} \beta_{12} \beta_{13} \beta_{14}=0.623
$$




\section{Group piles: $\boldsymbol{p}$-multipliers}

Mokwa (1999)

$f_{L}=0.64+0.06\left(\frac{s_{p}}{B}\right) \leq 1 \quad$ for the leading row

$f_{T 1}=0.34+0.11\left(\frac{s_{p}}{B}\right) \leq 1 \quad$ for the 1st trailing row

$f_{T 2}=0.16+0.14\left(\frac{s_{p}}{B}\right) \leq 1 \quad$ for the 2nd trailing row

$f_{\text {Тз }}=0.04+0.16\left(\frac{s_{p}}{B}\right) \leq 1 \quad$ for the 3rd and subsequent trailing row 


\section{Group piles: $\boldsymbol{p}$-multipliers}

Mokwa (1999)

$f_{L}=0.64+0.06\left(\frac{s_{p}}{B}\right)=0.64+0.06(3)=0.82 \quad$ for the leading row

$f_{T 1}=0.34+0.11\left(\frac{s_{p}}{B}\right)=0.34+0.11(3)=0.67$ for the 1st trailing row

Predicted $p$-multipliers

Leading Pile Trailing Pile

Reese et al (2006)

0.866

0.623

Mokwa (1999)

0.820

0.670 


\section{Group piles: comparison of p-multipliers}

- Comparison of predicted p-multipliers with measurements from pile group tests in dense, medium dense and loose sand

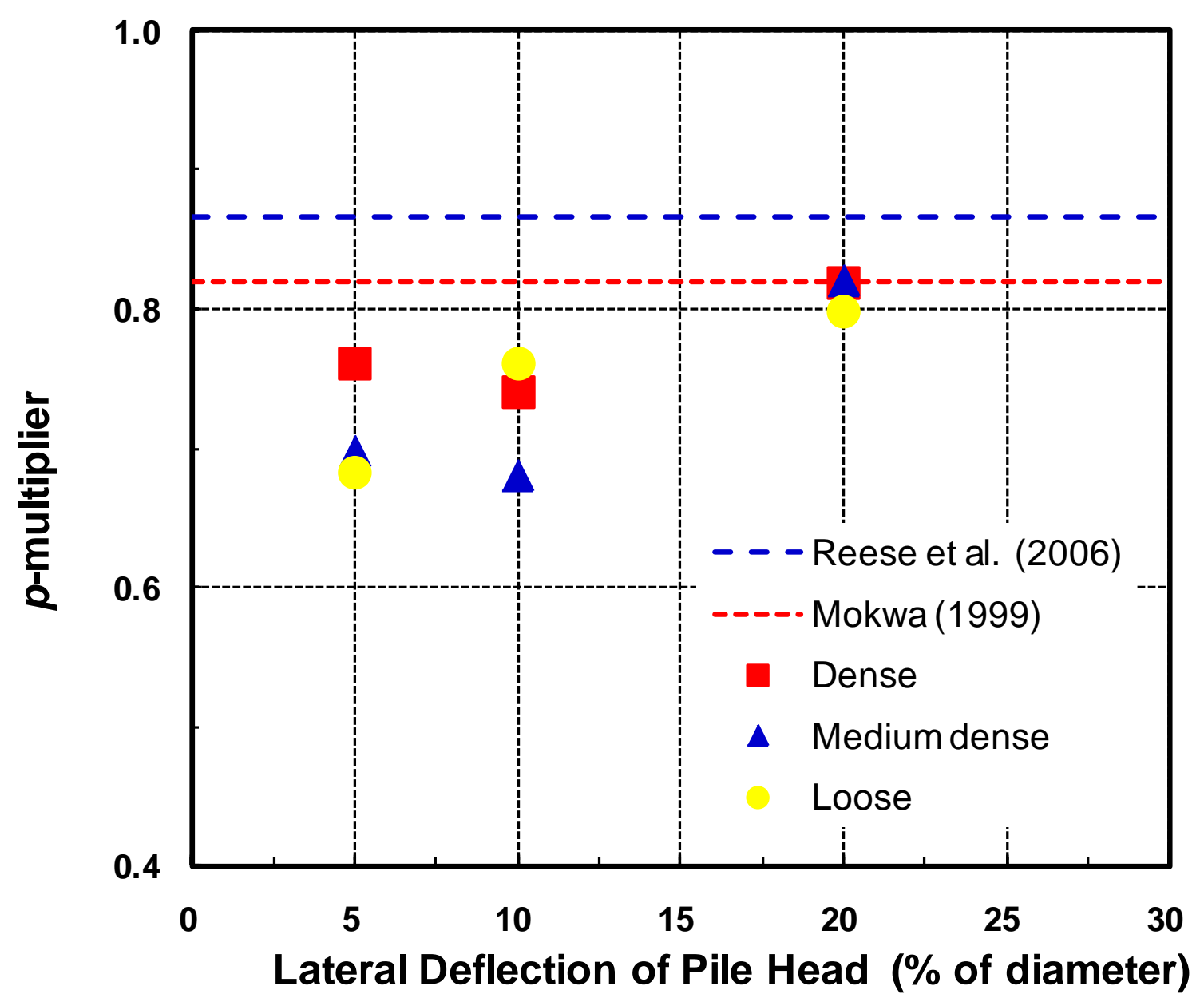




\section{Group piles: comparison of p-multipliers}

- Comparison of predicted p-multipliers with measurements from pile group tests in dense, medium dense and loose sand

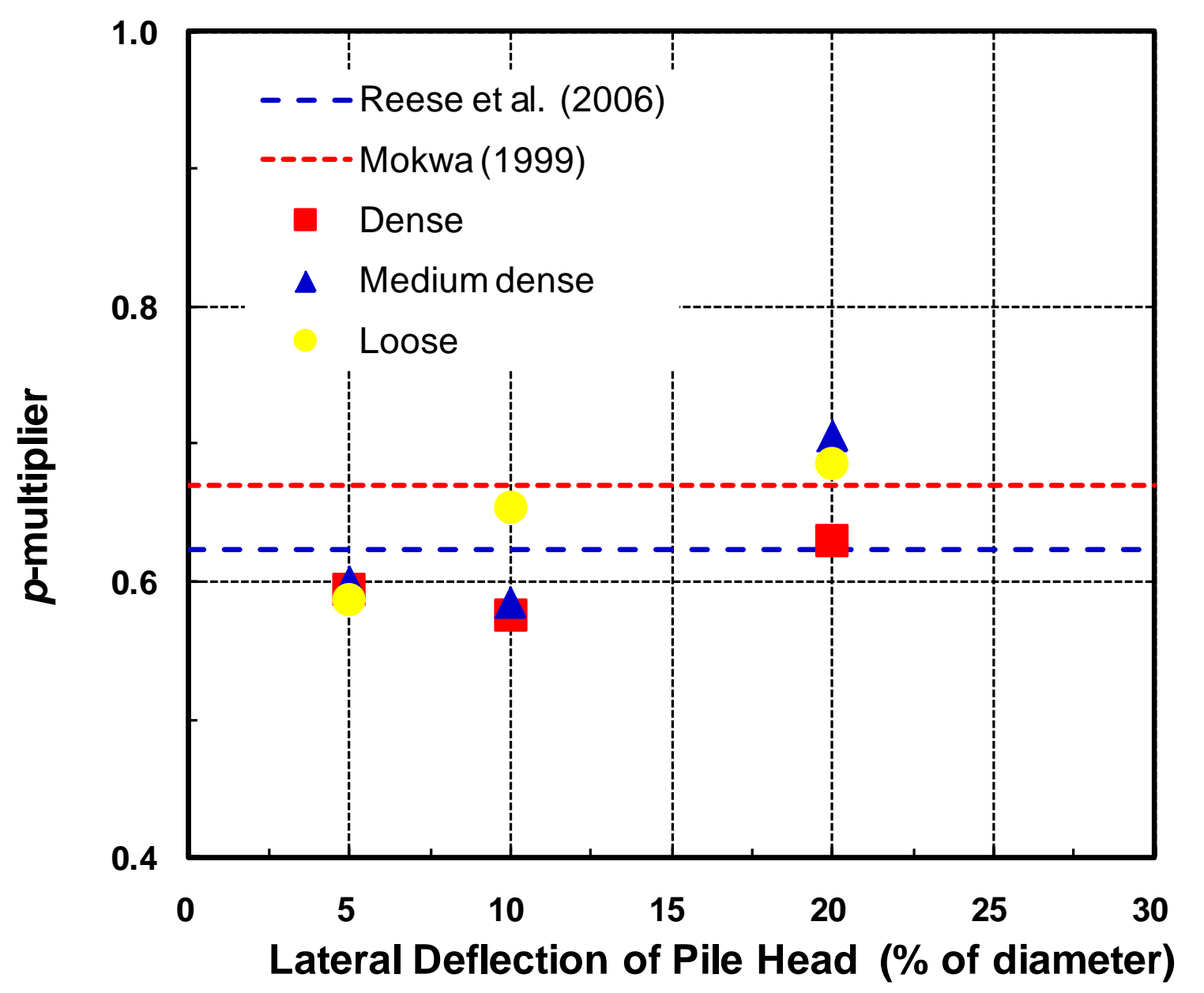




\section{Summary and Conclusions}

-An instrumented model pile, a soil tank, a large-scale pluviator, a driving system, and a jacking system were fabricated

- Lateral load tests were performed on preinstalled, driven and jacked model piles installed in sand prepared at different densities

- The effects of soil conditions and pile installation method on the model pile capacities were evaluated 


\section{Summary and Conclusions}

-For single piles, the predictions from the developed analysis were in good agreement with the model pile test results for small pile head deflections (up to $10 \%$ of the pile diameter)

-For pile groups, the measured $p$-multipliers are in reasonable agreement with those obtained from Mokwa (1999) and Reese et al. (2006)

- the measured $p$-multipliers for the leading piles were $85 \% \sim 90 \%$ less than the predicted values

- for the trailing piles, the measured $p$-multipliers were in good agreement with the predictions 


\section{References}

- Basu, D. (2006). Analysis of laterally loaded piles in layered soil. Ph.D. Thesis, Purdue University.

-Basu, D., Salgado, R. and Prezzi. M. (2008). "Analysis of laterally loaded piles in -multilayered soil deposits." Final Report FHWA/IN/JTRP-2007/23, JTRP -Lee, J. \& Salgado, R. (2000). Analysis of calibration chamber plate load tests. Can. Geotech. J., 37, 14-25.

- Mokwa, R. L. (1999). "Investigation of the resistance of pile caps to lateral loading." Ph.D. Thesis, Virginia Polytechnic Institute and State University.

- Mokwa, R. L. \& Duncan, J. M. (2001). Experimental evaluation of lateral-load resistance of pile caps. J. Geotech. Geoenv. Engng., Am. Soc. Civ. Engrs. 127, No. 2, 185-192.

- Poulos, H. G. \& Davis, E. H. (1980). Pile foundation analysis and design. John Wiley \& Sons, Inc.

- Randolph, M. F. (1981). The response of flexible piles to lateral loading. Geotechnique 31, No. 2,247-259.

- Reese, L. C., Isenhower, W. M. \& Wang, S.-T. (2006). Analysis and design of shallow and deep foundations. John Wiley \& Sons, Inc.

- Reese, L.C. and Van Impe, W.F. (2001). "Single Piles and Pile Groups Under Lateral Loading", A. A.Balkema, Rotterdam.

- Salgado, R. (2008). "The Engineering of Foundations", McGraw-Hill Science. 